University of Louisville

ThinkIR: The University of Louisville's Institutional Repository

Electronic Theses and Dissertations

$12-2011$

\title{
Optimization models for patient allocation during a pandemic influenza outbreak.
}

Li Sun

University of Louisville

Follow this and additional works at: https://ir.library.louisville.edu/etd

\section{Recommended Citation}

Sun, Li, "Optimization models for patient allocation during a pandemic influenza outbreak." (2011). Electronic Theses and Dissertations. Paper 1405.

https://doi.org/10.18297/etd/1405

This Doctoral Dissertation is brought to you for free and open access by ThinkIR: The University of Louisville's Institutional Repository. It has been accepted for inclusion in Electronic Theses and Dissertations by an authorized administrator of ThinkIR: The University of Louisville's Institutional Repository. This title appears here courtesy of the author, who has retained all other copyrights. For more information, please contact thinkir@louisville.edu. 


\title{
OPTIMIZATION MODELS FOR PATIENT ALLOCATION DURING A PANDEMIC INFLUENZA OUTBREAK
}

\author{
By \\ Li Sun
}

\begin{abstract}
A Dissertation
Submitted to the Faculty of the

J. B. Speed School of Engineering of the University of Louisville in Partial Fulfillment of the Requirements

for the Degree of

Doctor of Philosophy

Department of Industrial Engineering

University of Louisville

Louisville, Kentucky
\end{abstract}

December 2011 


\title{
OPTIMIZATION MODELS FOR PATIENT ALLOCATION DURING A PANDEMIC INFLUENZA OUTBREAK
}

\author{
By
}

Li Sun

A Dissertation Approved on

December 9, 2011

By the following Dissertation Committee:

$\overline{\text { Dissertation Director: Dr. Gail bePuy }}$

Dr. Sunderesh Heragu

Dr. Gerald Evans

Dr. William Biles

Dr. ScottLajoie 


\section{ACKNOWLEDGEMENTS}

I would never have been able to finish my dissertation without the guidance from my advisor and committee members, help from RTDSS research team members, and support from my friends and family.

I am heartily thankful to my dissertation advisor, Professor Gail DePuy. Her guidance and inspiration along the way were so helpful and made this dissertation possible. Her patience and encouragement always gave me confidence when I lost it. Her understanding and consideration also made working with her enjoyable. I would also like to thank Dr. Heragu. He gave me this great opportunity to be involved in this concrete and exciting project. His insightful suggestions and comments helped to improve my work. I would also like to thank Dr. Evans, Dr. Biles and Dr. LaJoie. They have generously given their time and expertise to better my work.

I am grateful to RTDSS research team members. I thank them for their contribution and their good-natured support. My research would not have been possible without their helps. Special thanks go to Dr. Kelley and Phani, who developed the web-application for my work.

It is a pleasure to thank my dear friends. Their friendship makes life much happier and more colorful. I owe my deepest gratitude to my dear great parents for their love and support. 


\section{ABSTRACT \\ OPTIMIZATION MODELS FOR PATIENT ALLOCATION DURING A PANDEMIC INFLUENZA OUTBREAK}

\section{Li Sun}

\section{December 9, 2011}

Pandemic influenza has been an important public health concern. During the 20th century, three major pandemics of influenza occurred in 1918, 1957, and 1968. The pandemic of 1918 caused 40 to 50 million deaths worldwide and more than 500,000 deaths in the United States. The 1957 pandemic, during a time with much less globalization than now, spread to the U.S. within 4 to 5 months of its origination in China, causing more than 70,000 deaths in the U.S., and the 1968 pandemic spread to the U.S. from Hong Kong within 2 to 3 months, causing 34,000 deaths.

Pandemic influenza is considered to be a relatively high probability event, even inevitable by many experts. During a pandemic influenza outbreak, some key preparedness tasks cannot be accomplished by hospitals individually; regional resource allocation, patient redistribution, and use of alternative care sites all require collaboration among hospitals both in planning and in response. The research presented in this dissertation develops optimization models to be used by decision makers (e.g. hospital associations, emergency management agency, etc.) to determine how best to manage 
medical resources as well as suggest patient allocation among hospitals and alternative healthcare facilities.

Both single-objective and multi-objective optimization models are developed to determine the patient allocation and resource allocation among healthcare facilities. The single-objective optimization models are developed to optimize the patient allocation in terms of minimizing the travel distance between patients and healthcare facilities while considering medical resource capacity constraints. During the pandemic, the surge demand most likely would exhaust all the medical resources, at which time the models can help predict the potential resource shortage so an appropriate contingency plan can be developed. If additional resource quantities become available, the models help to determine the best allocation of these resources among healthcare facilities. Various methods are proposed to conduct the sensitivity analysis to help decision makers determine the impact of different level of each type resource on the patient service. The multi-objective optimization model not only considers the objective of minimization of the total travel distance by patients to healthcare facilities, but also considers the minimization of maximum patient travel distance. A case study from Metro Louisville, Kentucky is presented to demonstrate how the models would aid in patient allocation and resource allocation during a pandemic influenza outbreak. A web-based application based on the optimization models developed in this dissertation is presented as an initial tool for decision makers. 


\section{TABLE OF CONTENTS}

ACKNOWLEDGEMENTS .......................................................................... iii

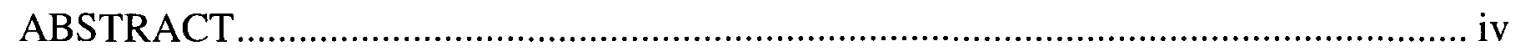

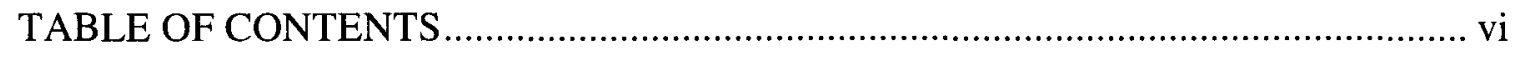

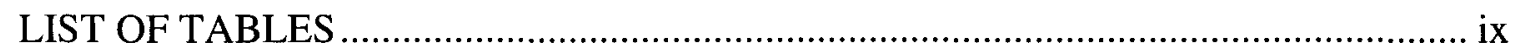

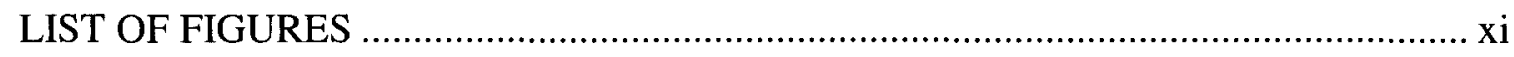

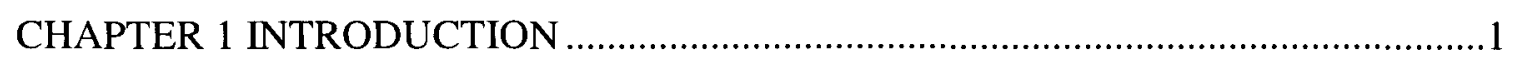

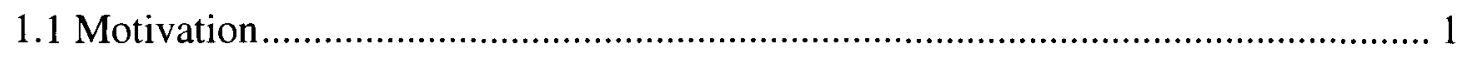

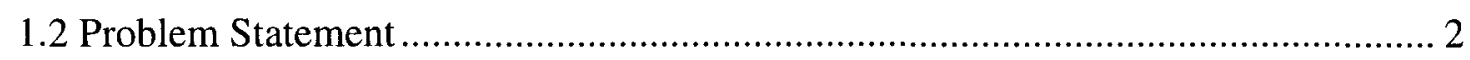

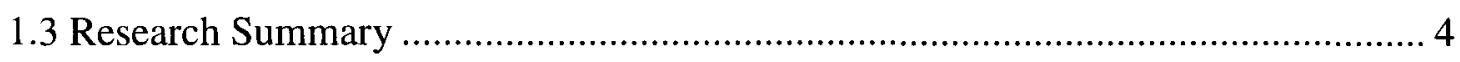

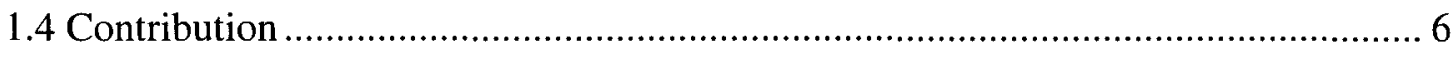

1.5 Dissertation Organization ......................................................................... 7

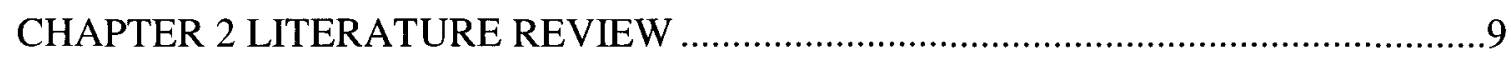

2.1 Models for Pandemic Influenza Planning/Response ...................................... 9

2.2 Models for the Allocation Problem in a Hospital Network ................................... 12

2.2.1 Long Term Allocation Model ................................................................. 13 
2.3 Multi-Objective Optimization Solution Methodologies ................................ 16

2.3.1 Multiple Objectives Combined into Single Function ................................. 17

2.3.2 Determine a Set of Efficient Solutions for Multi-Objective Optimization...... 20

CHAPTER 3 SINGLE OBJECTIVE OPTIMIZATION MODELS ...........................23

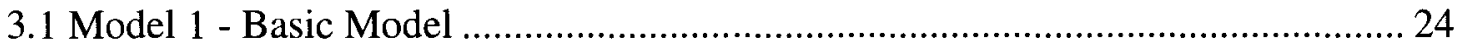

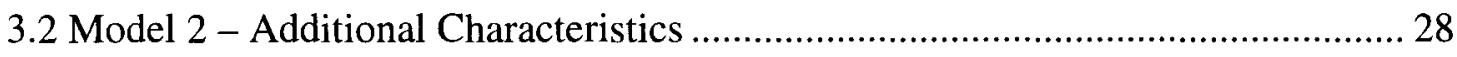

3.3 Model 3 - Model for Multiple Planning Horizons ...................................... 32

3.4 Model 4 - Model for Multiple Planning Horizons and Allowed Resource Shortages 37

3.5 Model 5 - Model Considering the Allocation of Additional Resources ............... 41

CHAPTER 4 CASE STUDY AND SENSITIVITY ANALYSIS ...............................43

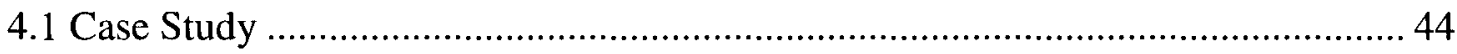

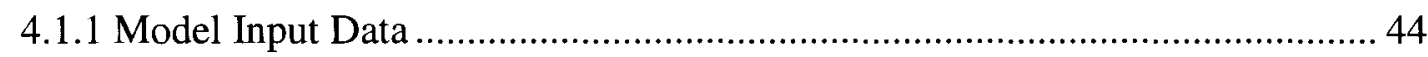

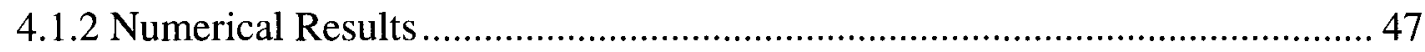

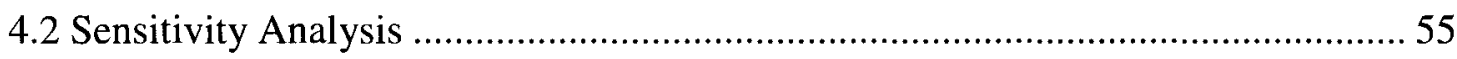

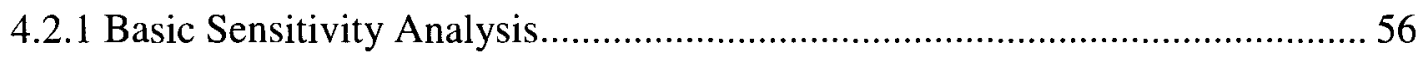

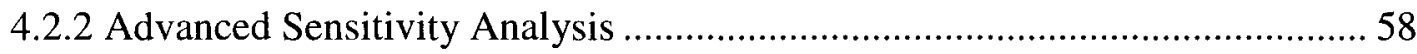

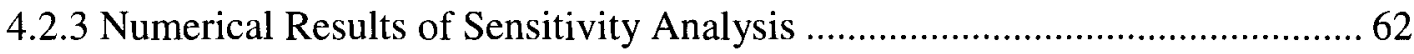

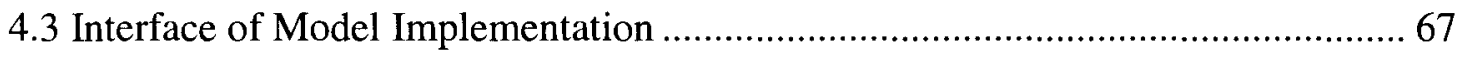


5.1 Multi-Objective Patient Allocation Model ...................................................... 74

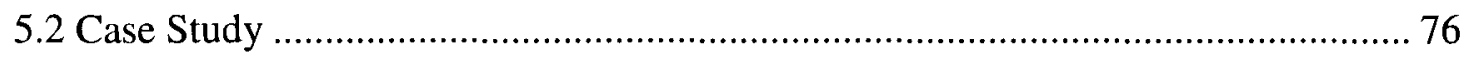

CHAPTER 6 CONCLUSIONS AND FUTURE STUDIES ………….............................

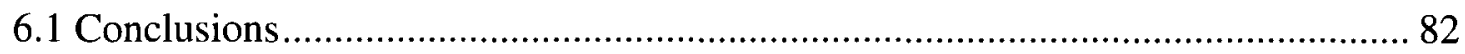

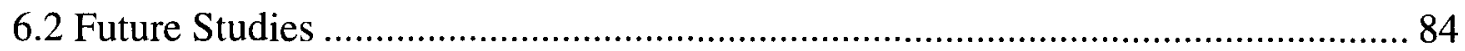

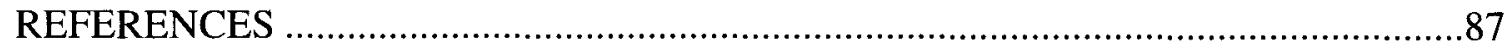

APPENDIX

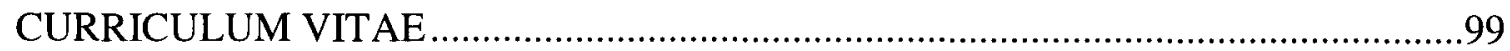

viii 


\section{LIST OF TABLES}

Table 1. Model 1 runtime for various data set sizes .................................................... 28

Table 2. Distance (in miles) between population areas and hospitals .......................... 45

Table 3. Total number of each type resource at hospitals...................................... 47

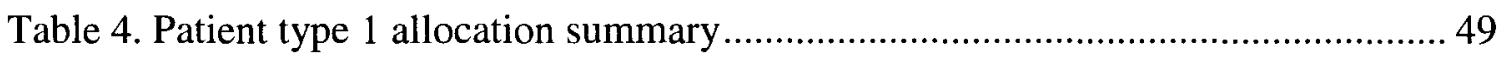

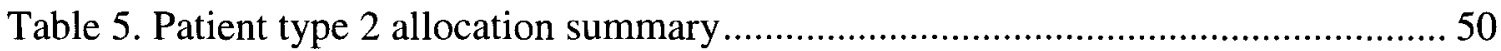

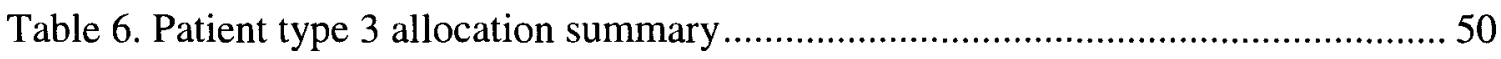

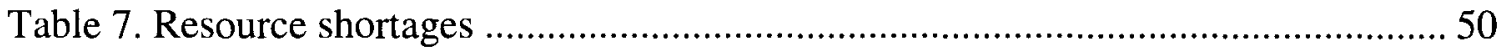

Table 8. Allocation of additional resources ........................................................... 51

Table 9. Patient type 1 allocation summary with additional resource allocation .............51

Table 10. Patient type 2 allocation summary with additional resource allocation .......... 51

Table 11. Patient type 3 allocation summary with additional resource allocation ...........52

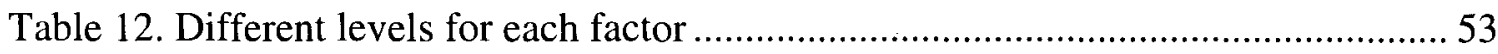

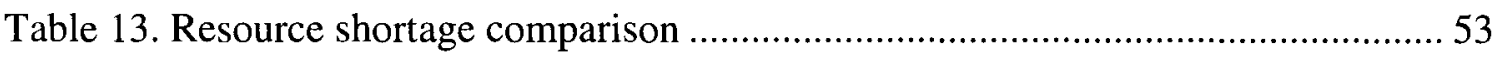

Table 14. Sensitivity analysis report from LINGO.............................................. 58

Table 15. Lower bound and upper bound of each type resource level ....................... 63

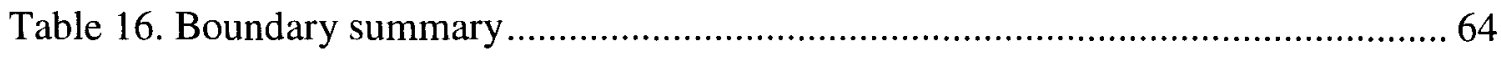

Table 17. Computer run times for sensitivity analysis methods .............................. 66

Table 18. Patient type 1 allocation summary for single objective ........................... 77 
Table 19. Distance (miles) for the single objective of minimization of total travel distance 78

Table 20. Patient type 1 allocation summary for multi-objective patient allocation model. 79

Table 21. Distance (miles) for multi-objective patient allocation model. 80

Table 22. Demand estimation for 6-week pandemic duration, 35\% gross attack rate, most likely scenario (day 1-14) 92

Table 23. Demand estimation for 6-week pandemic duration, 35\% gross attack rate, most likely scenario (day 15-28) 93

Table 24. Demand estimation for 6-week pandemic duration, 35\% gross attack rate, most likely scenario (day 29-42) 94

Table 25. Allocation of patient type 1 for 6-week pandemic duration, 35\% gross attack rate, most likely scenario (day 1-14) 95

Table 26. Allocation of patient type 2 for 6 -week pandemic duration, $35 \%$ gross attack rate, most likely scenario (day $1-14)$ 96

Table 27. Allocation of patient type 3 for 6 -week pandemic duration, $35 \%$ gross attack rate, most likely scenario (day 1-14) 96

Table 28. Allocation of patient type 1 with additional resource allocation for 6-week pandemic duration, $35 \%$ gross attack rate, most likely scenario (day 1-14) 97

Table 29. Allocation of patient type 2 with additional resource allocation for 6-week pandemic duration, $35 \%$ gross attack rate, most likely scenario (day 1-14) 97 Table 30. Allocation of patient type 3 with additional resource allocation for 6-week pandemic duration, $35 \%$ gross attack rate, most likely scenario (day 1-14) 98 


\section{LIST OF FIGURES}

Figure 1. Map of Metro Louisville, Kentucky .................................................... 45

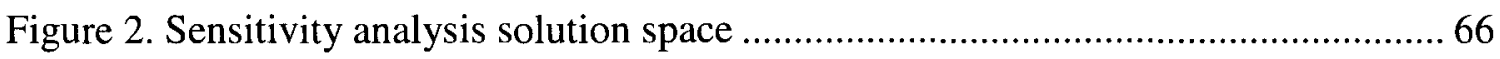

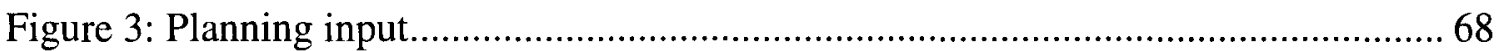

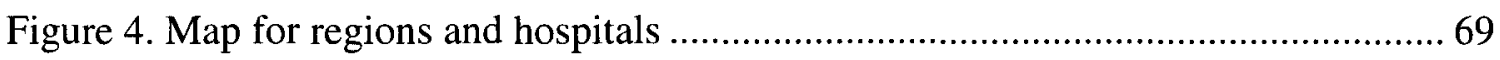

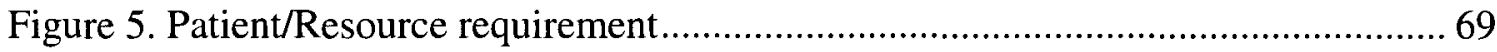

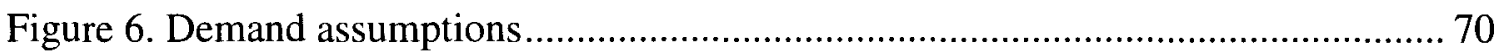

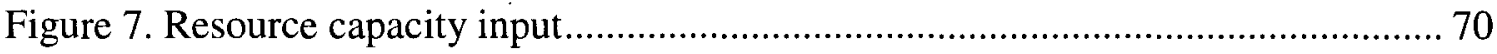

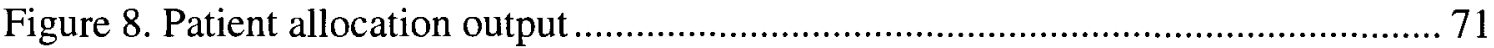

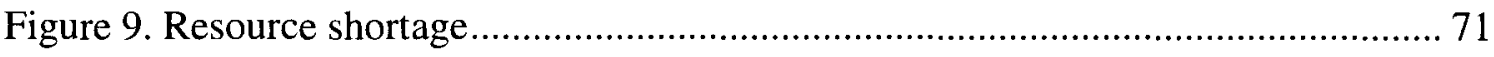

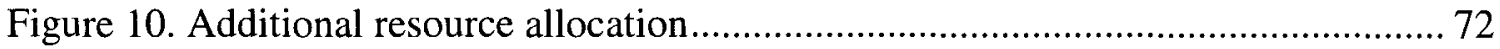




\section{CHAPTER 1 INTRODUCTION}

\subsection{Motivation}

Pandemic influenza has been an important public health concern. During the 20th century, three major pandemics of influenza occurred in 1918, 1957, and 1968. The pandemic of 1918 caused 40 to 50 million deaths worldwide and more than 500,000 deaths in the United States. The 1957 pandemic, during a time with much less globalization than now, spread to the U.S. within 4 to 5 months of its origination in China, causing more than 70,000 deaths in the U.S., and the 1968 pandemic spread to the U.S. from Hong Kong within 2 to 3 months, causing 34,000 deaths (Das et al., 2008; Meltzer et al., 1999).

Pandemic influenza is considered to be a relatively high probability event, even inevitable by many experts (Ekici et al., 2008). Many researchers study the impact of the next pandemic. Based on extrapolation of the 1957 and 1968 pandemic, the Centers for Disease Control and Prevention (CDC) estimates that in the U.S. there could be 839,000 to $9,625,000$ hospitalizations, 18 to 42 million outpatient visits, and 20 to 47 million additional illnesses, depending on the attack rate of infection during the pandemic (Meltzer et al., 1999). The estimates based on the more severe 1918 pandemic suggest that substantially more hospitalizations and deaths could occur (Meltzer et al., 1999). A software program called FluSurge was developed by the CDC to calculate the potential 
impact of a pandemic on hospital resources such as staffed beds (both overall and ICU Intensive Care Unit) and ventilators (Zhang et al., 2006). The results from FluSurge indicate that hospitals would be severely stressed in the moderate 1968-like scenario, and completely overwhelmed in the case of a severe 1918-like pandemic (Toner \& Waldhorn, 2006). Toner and Waldhorn (2006) state that during a pandemic influenza outbreak, some key preparedness tasks cannot be accomplished by hospitals individually, and it is necessary that regional resource allocation, patient redistribution, and use of alternative care sites all require collaboration among hospitals both in planning and in response. The research presented in this dissertation develops optimization models to be used by decision makers (e.g. hospital association, emergency management agency, etc.) to determine how best to manage medical resources as well as suggest patient allocation among hospitals and alternative healthcare facilities.

\subsection{Problem Statement}

This research focuses on how to allocate the inpatients among the hospitals and other healthcare facilities during a pandemic influenza outbreak. Previous work related to patient allocation mostly focuses on either long term planning such as hospital network planning over a span of years (Chu \& Chu, 2000; Green et al., 1980; Gunes \& Yaman, 2010; Harper et al., 2005; Mitropoulos et al., 2006; Ruth, 1981; Santibanez et al., 2009; Schweikhart \& Smith-Daniels, 1993; Stummer et al., 2004; Syam \& Côté, 2010) or short term planning such as emergency disaster response to a earthquake or hurricane in a matter of hours or days (Fiedrich et al., 2000; Minciardi et al., 2009; Yi \& Ozdamar, 2007). Since a pandemic outbreak usually lasts several months, it is considered to be a medium term planning problem. In this research several mathematical models are 
formulated and solved to help decision makers address the patient and resource allocation issues faced by a multi-facility healthcare network in a medium term influenza outbreak. The mathematical models developed in this research optimize the patient allocation in terms of:

1) Minimizing the patients" "cost" of access to service measured as the total travel distance between patients and healthcare facilities;

2) Minimizing the maximum patient travel distance;

3) Optimizing patient preferences such as special service for certain patients; for example patients prefer traveling farther to primary hospital other than health center, patients prefer particular healthcare facility that accepts their insurance;

4) Balancing the workload (i.e. the number of patients treated) among healthcare facilities via a predefined desirable occupancy rate;

5) Satisfying resource capacities via constraints to incorporate limited availability of resources at each facility. Resources can include the physical facilities (such as non ICU-beds, ICU-beds, and ventilators) and health-care personnel (such as doctors, nurses, and lab technicians). Various patient types are considered, requiring different levels of service based on the severity of their illness. Each patient type category requires multiple types of resources and occupies the resources for a specific time period depending on the length of stay in the hospital, which is incorporated into the capacity constraints.

An initial mathematical model is developed to predict when (i.e. which day), where (i.e. which healthcare facility) and what (i.e. which resource) is exceeded by the influenza patient demand during the outbreak. Based on these predictions, decision makers can 
determine if healthcare facilities need to increase medical capacity (e.g., have personnel work extra hours or put patients in hallways) or request additional capacity from the state or national stockpile, which is designed to supplement and re-supply healthcare facilities and state/local public health agencies in the event of an emergency. If the healthcare facilities receive new resources and/or agree to transfer the resources among healthcare facilities, the model can be used to optimize the allocation of resources.

The mathematical models are also used to perform 'what if ...' scenario analyses to evaluate the system performance under different scenarios, such as considering different amounts of new resources, different demand levels, and different policies to transfer resources among healthcare facilities.

\subsection{Research Summary}

This research is focused on the admitted or in-patient allocation and resource allocation to decide which healthcare site the patients should go to, no matter how they are transported; either patients arrive to the healthcare site by themselves or are delivered by EMS (Emergency Medical Services). The results from the models can help:

1) To encourage patients to go to specific healthcare sites, which can be implemented in the following ways:

a. Family physicians refer respective patients to appropriate hospitals or other alternative healthcare facilities;

b. To increase public awareness of each healthcare facility's availability: developing the dashboard website, or through phone; 
c. EMS delivers patients to appropriate hospitals or other alternative healthcare facilities;

d. Other public policy?

2) To predict the utilizations of various resources among healthcare facilities over time, which would help the healthcare facility to be well prepared;

3) To predict when (which day) where (which healthcare facility) and what (which resource) capacity would be exceeded by the surge demand, therefore the surge capacity needs would be identified;

4) To allocate the new resources among healthcare facilities, and/or transfer the existing resources among healthcare facilities.

The state/local hospital association, EMS, and healthcare emergency response agents can apply the models to determine the patient distribution and potential resource allocation among the healthcare network during an influenza pandemic outbreak.

This research focuses on medium-term planning during a pandemic influenza outbreak, which is different from either long-term or short-term planning as in most current research, in the following ways:

- The planning horizon can be divided into several shorter planning horizons to reduce the solution run time and to allow the interjection of real changes in the system during the disease spread such as surge capacity increase and healthcare personnel infection during the pandemic development. Most current research models utilize a single planning horizon; 
- The model will consider the length of stay of patients in hospitals or other alternative healthcare facilities, which is included explicitly in the capacity constraints, while most current research models simplify the stay of length by estimating a general capacity rate, such as number of patients per time unit (Gunes \& Yaman, 2010; Santibanez et al., 2009; Stummer et al., 2004);

- Sensitivity analysis is applied to optimization models and helps determine how different levels of additional resource impact the service to the patients;

- A multi-objective optimization model will be developed to consider two objectives related to patients' cost of access to healthcare services, including minimization of the total travel distance by patients to service and minimizing the maximum distance a patient travels.

\subsection{Contribution}

The contribution of this work is summarized as follows.

- Problem:

This work is problem-focused. Much work has been done related to pandemic influenza response, as discussed in following literature review Chapter 2. But as can best be determined, the patient allocation along with resource allocation problems during an influenza pandemic outbreak has not been studied in the literature. In addition, sensitivity analysis is applied to optimization models which helps determine how different levels of additional resource in a healthcare network impact the service to the patients.

- Models: 
As mentioned, this work is a medium term planning problem, while most the allocation models in a healthcare setting focus on either long term planning or short term planning. In this work, the models consider the length of stay of patients in healthcare facilities explicitly in the capacity constraint, while most current research models simplify the stay of length by estimating a general capacity rate. In addition, the planning horizon can be divided into several shorter planning horizons to reduce the solution run time and to allow the interjection of real changes in the system during the disease spread such as surge capacity increase and healthcare personnel infection during the pandemic development, and the models are developed to connect consecutive shorter planning horizons with one another. Most current research models utilize a single planning horizon. Finally, a dummy hospital is included into the models to generate feasible solutions when encountering resource shortages in the healthcare network.

- Results and implementation:

The models generate optimal solutions in a reasonable run time for various scales, considering it is a medium term planning problem. A web-application tool, based on the models in this work, is developed by a research team. The users are allowed to solve the models and obtain results in a reasonable time through the website. Users can determine the patient allocation, predict the potential resource shortage, and decide the allocation of additional resources among the healthcare facilities during an influenza pandemic outbreak. The users also can apply what-if analysis to evaluate the system performance under various situations.

\subsection{Dissertation Organization}


The remainder of the dissertation is organized as follows:

In Chapter 2, a comprehensive literature review is presented, including the literature related to pandemic influenza planning and response, literature related to healthcare allocation modeling for normal, non-pandemic instances, and literature related to the solution techniques. In Chapter 3, the formulations of several single objective optimization models are presented. The objective of these models is to minimize the patients' cost (i.e. travel distance) to the service. In Chapter 4, numerical results for a case study from Metro Louisville, $\mathrm{KY}$ are presented to demonstrate how the model can help decision makers determine the patient allocation and potential resource shortages in the healthcare system, as well as help to decide the potential surge medical resource allocation. In addition, sensitivity analysis is applied to the single-objective model, which is mainly used to help decide how different respective levels of additional resources would improve the service to the patients. A web-based version of the optimization models developed in this dissertation was implemented by a team of web developers. An example of this web-based tool is included in Chapter 4. In Chapter 5, multi-objective optimization models are formulated to optimize the patient allocation and potential surge medical resource allocation among the healthcare sites considering two objectives related to patients' cost of access to healthcare services: 1) minimization of the total travel distance by patients to healthcare sites; and 2) minimization of the maximum travel distance of a patient. Numerical results are presented for a case study. Finally in Chapter 6 , the conclusions and future study plans for the research are presented. 


\section{CHAPTER 2 LITERATURE REVIEW}

This literature review divides the relevant literature into three categories: literature related to pandemic influenza planning and response, literature of healthcare allocation modeling for normal, non-pandemic instances, and literature related to the solution techniques proposed in this work.

\subsection{Models for Pandemic Influenza Planning/Response}

Pandemic influenza planning/response involves various aspects, including, forecasting of the disease spread, mass vaccination clinics, and current tools available for hospital planning in the event of a pandemic influenza outbreak.

Disease spread modeling is used to estimate the infectious disease progression, which helps to prepare for the pandemic influenza outbreak. Various researchers use mathematical or simulation models to estimate the disease spread (Bobashev et al., 2007; Larson, 2007; Naron \& Wasserkrug, 2007; Parker, 2007). Based on the disease spread model, a growing amount of literature studies the impact of a variety of mitigation strategies, including vaccination, prophylaxis, social distance, quarantine, and travel restrictions. Difference scale models (such as local, regional, national, and global) are studied in the literature to investigate the spread of a pandemic influenza outbreak under various mitigation strategies (Chowell et al., 2006; Colizza et al., 2007; Das et al., 2008;

Eubank et al., 2004; Ferguson et al., 2005; Germann et al., 2006; Goedecke et al., 2007; 
Halloran et al., 2008; Jenvald et al., 2007; Kaplan et al., 2002; Lant et al., 2008; Longini Jr. et al., 2005; Wu et al., 2006).

Mass vaccination is one of the important mitigation strategies. Various papers focus on the logistics aspect of mass vaccination, such as the location problem of the point-ofdispensing (POD) facilities, and the resource allocation in the POD, which can be considered as a short term planning problem since the mass vaccination takes place in a short period (days or hours). A software suite of decision-support systems, RealOpt, for planning large-scale emergency dispensing clinics (i.e. point-of-dispense, POD) to responder to biological threats and infectious-disease outbreaks is developed by Lee and her group (Lee et al., 2009; Lee et al., 2006a, 2006b; Lee et al., 2009). The software contains a component to solve the POD-location problem, a simulation component to estimate the POD service performance (e.g. average wait time, average queue length, average utilization rate, etc.), and an optimization component to solve the resource allocation problem in the POD. Another software package containing discrete-event simulation models and capacity-planning and queueing-system models is developed by (Aaby et al., 2006; Aaby et al., 2006) to plan emergency mass dispensing and vaccination clinics for Montgomery County's (Maryland) Public Health Services (PHS).

Hospital planning during a pandemic influenza outbreak is another important area in the literature. The Centers for Disease Control and Prevention (CDC) of the U.S. Department of Health and Human Services provides pandemic influenza response tools FluAid 2.0 and FluSurge 2.0. FluAid 2.0 is designed to assist state and local level planners in preparing for an influenza pandemic by providing a range of estimates of potential impact in terms of deaths, hospitalizations and outpatient visits due to pandemic influenza 
(Meltzer et al., 2000). FluSurge 2.0 is a spreadsheet-based model to allow hospital administrators and public health officials to access the impact of pandemic influenza on hospital services (Zhang et al., 2005).

FluSurge 2.0 users are asked to provide estimates of their local population in three age groups $(0-19,20-64$, and $65+$ years) and their total hospital resources (total non-ICU hospital beds, total ICU beds, and total mechanical ventilators), and are able to select the duration of a pandemic and the gross clinical attack rate. Based on these data, FluSurge estimates the number of hospital admissions and deaths due to pandemic influenza and compares hospital resources needed during a pandemic with existing hospital resources.

A number of researchers applied FluAid and FluSurge to study pandemic responses in various geographical areas. For example, Zhang et al., (2006) discuss Metropolitan Atlanta to demonstrate the results, using population-based rates of illness and death in an influenza pandemic adapted from Meltzer et al., (1999). Sobieraj et al. (2007) use FluSurge 2.0 to determine hospital capabilities at William Beaumont Army Medical Center (WBAMC) in response to patient arrival surges of the Fort Bliss population in mild 1968-type and severe 1918-type influenza pandemics. Lum et al. (2009) apply FluSurge 2.0 and FluAid 2.0 to estimate the demand for critical care hospital admissions in Victoria, Canada resulting from the rapid rise in the number of pandemic (H1N1) 2009 influenza cases, and compare the estimation with the data obtained from daily hospital reports of pandemic (H1N1) 2009 influenza-related admissions and transfers to intensive care units (ICUs). The results show that the observed rate of hospital admissions was broadly consistent with a $5 \%$ gross clinical attack rate, with $0.3 \%$ of infected patients being hospitalized and $20 \%$ transfer rate of hospital admissions to ICUs. They conclude 
that the prospective use of modelling tools informs critical decisions in the planning and management of the pandemic and early estimation of the clinical attack rate, hospitalization rates, and demand for ICU beds guides implementation of surge capacity.

Rico et al. (2007) present a simulation model to optimize nurse allocation in a Veteran's Hospital during a pandemic influenza outbreak by using FluSurge 2.0 to determine the arrival rate of patients to the hospital. Wilson, et al. (2005) apply FluAid to estimate the impact of the next influenza pandemic on population health and health sector capacity in New Zealand. Menon et al. (2005) use FluSurge to model the impact of an influenza pandemic on critical care services in England. It should be noted that all the literature regarding using FluAid and FluSurge for hospital planning focuses on one hospital, not a hospital network, or simply compares the existing hospital resources with the estimated demand, not related to the collaboration among hospitals in a network. It appears that the patient allocation and resource allocation among a healthcare network of hospitals or other alternative healthcare facilities during a pandemic influenza has not been studied.

\subsection{Models for the Allocation Problem in a Hospital Network}

The literature related to the allocation problem (i.e. patient allocation, resource allocation/re-allocation) in a hospital network is reviewed here. Previous work mostly focuses on either long term planning (e.g. hospital network planning for future years) or

short term planning (e.g. ambulance allocation, and emergency disaster response). This research deals with a medium-term planning horizon, considering that a pandemic influenza usually lasts several months, neither years nor days. 


\subsubsection{Long Term Allocation Model}

The location-allocation model that aims to simultaneously determine optimal facility locations and the assignment of customers to the selected facilities has been extensively applied in healthcare settings to decide the location of healthcare facilities and the assignment of patients to facilities. Various researchers apply the location-allocation model to design a hospital network in a long term planning horizon. These models are used by decision makers to determine the resource allocation/re-allocation among hospitals, the assignment of patients to hospitals, the service portfolio (i.e. if a service is placed in a hospital or not), and the location of a healthcare facility.

Gunes and Yaman (2010) present a mixed integer programming formulation for replanning hospitals after a merger of Turkish hospital networks. The model is used to to optimize the re-allocation of resources (i.e. beds and specialist doctors) among hospitals, the assignment of patients to hospitals and the service portfolio to minimize the systems costs subject to quality and capacity constraints.

Santibanez et al. (2009) use a mixed integer programming model to plan Fraser Health's, British Columbia's largest regional health authority, hospital network to increase its acute care capacity over the next 15 years ( 3 planning periods are considered in the planning horizon) for its anticipated population growth and aging. They formulate a facility location-allocating model to determine if a service should be placed in a hospital or not, in every decision period, and to assign demand for a service from each community to the hospitals in each decision period. 
Harper et al. (2005) develop a discrete-event geographical location-allocation simulation model to evaluate various options for the provision of healthcare services, which includes the location of the service centers, service capacities, and patients' access to the service centers. Their stochastic location-allocation approach considers various patient flows, traveling times, and transport preferences.

Syam and Côté (2010) develop a mixed integer programming model to solve the location and allocation of specified healthcare services such as traumatic brain injury (TBI) treatment in one of the Department of Veterans Affairs' (VA) integrated service networks.

Stummer et al. (2004) develop a multi-objective combinatorial optimization model to determine location and size of medical departments within the hospitals of a given network.

Ruth (1981) develops a mixed integer programming model for regional planning of a hospital inpatient service. The model is used to solve the allocation of beds to match the demand. It needs to minimize the cost of the changes considering a distance constraint to ensure geographical accessibility, which makes sure patients requiring a certain level service should be expected to travel within an acceptable distance.

Mitropoulos et al. (2006) develop a bi-objective model for the location planning of hospital and health centers to minimize the total weighted traveling distance from population centers to the healthcare facilities, and to minimize the maximum distance from population centers to the medical facilities. 
Schweikhart and Smith-Daniels (1993) present a nonlinear integer model to determine the number, location and service offerings of facilities for a managed healthcare network in order to minimize cost and maximize market share.

Chu and Chu (2000) present a modeling framework to plan for the supply and demand matching of public hospital beds in Hong Kong, which addresses 1) hospital locations and 2) service allocations, including new services distribution as well as existing services redistribution.

Green et al. (1980) use the bounded transportation problem to investigate new hospital location and optimal patient assignments in rural Appalachian Ohio hospitals.

Various researchers study resource allocation in a healthcare setting as well. One of the problems focuses on monetary allocation. For example, Zaric and Brandeau (2001) present a model for allocation of epidemic control resources among a set of interventions, aiming to maximize quality-adjusted life years gained or the number of new infections averted over a fixed time horizon, subject to a budget constraint. Koyuncu and Erol (2010) develop a multi-objective model for optimal resource allocation (monetary budget for antivirals and preventive vaccinations, ICU beds, ventilators and non-ICU beds) to minimize the deaths, number of cases and total morbidity days during a pandemic influenza. Flessa (2003) analyzes the optimal allocation of monetary budget to preventive programs and the four levels of curative care in the Mtwara region, Tanzania.

The allocation of available hospital resources to a service is another problem in healthcare setting. Govind, Chatterjee, and Mittal (2008) examine a network of hospitals in a predefined geographical area to determine the resources (specifically, beds) that each 
hospital in the network should devote to different disease classes in order to maximize speed of access to care. Blake and Carter (2002) present linear goal programming models for allocating resources to decide the case mix and volume for physicians in hospitals.

\subsubsection{Short Term Allocation Model}

Various researchers study emergency response over a short time horizon, such as after an earthquake, hurricane, or terrorist attack. The planning horizon could be days, or even hours in the disaster response situation. Fiedrich et al. (2000) study the assignment of available resources to operational areas in order to minimize the total number of fatalities during the initial search-and-rescue period after strong earthquakes, using a dynamic combinatorial optimization model. Yi and Ozdamar (2007) build an integrated locationdistribution model to study the selection of temporary emergency centers that result in maximum coverage of medical need in affected areas after disasters, and the optimal medical personnel allocation among both temporary and permanent emergency units. Minciardi et al. (2009) develop a mathematical model to support optimal resource allocation before and during an emergency due to natural hazard events.

\subsection{Multi-Objective Optimization Solution Methodologies}

Multiple objectives will be considered in the future study to optimize the allocation of the patients and potential additional resources among hospitals and other alternative healthcare facilities. Hence, the multi-objective optimization solution methodologies and the application in the healthcare setting are reviewed.

Multi-objective optimization involves the optimization of a collection of objectives. The general multi-optimization problem is defined as follows (Marler \& Arora, 2004): 
Minimize $F(X)=\left[F_{1}(X), F_{2}(X), \ldots, F_{k}(X)\right]^{T}$

Subject to $g_{j}(X) \leq 0, j=1,2, \ldots, m$,

$h_{l}(X)=0, l=1,2, \ldots, e$,

Where $\mathrm{k}$ is the number of objective functions, $\mathrm{m}$ is the number of inequality constraints, and $\mathrm{e}$ is the number of equality constraints. $\mathrm{X}$ is a vector of decision variables.

There are two general approaches for solving the multiple-objective optimization problem. One solution technique is to combine the multiple objectives to a single composite function or move all but one objective to the constraint set. The other approach is to generate a set of efficient solutions and allow the decision maker to evaluate the solutions and choose a desirable course of action.

\subsubsection{Multiple Objectives Combined into Single Function}

There are many ways to transform a multi-objective optimization to single-objective optimization problem, including weighted-sum approach, goal programming, and $\varepsilon$ constraint programming. Weighted-sum approach is one of most commonly-used classical methods (Deb, 2001). As the name suggests, the weighted-sum approach transforms a multi-objective optimization problem to a single-objective optimization problem by pre-multiplying each objective with a user-supplied weight (Deb, 2001). Then the objective is transformed to be:

Minimize $U=\sum_{i=1}^{k} w_{i} F_{i}(X)$ 
It is important to notice that this weighted-sum approach is highly sensitive to the predefined weight vector supplied by the decision maker. Finding a weight vector itself is highly subjective and not straightforward.

Santibanez et al. (2009) build a mathematical model to plan the inpatient hospital network for Fraser Health in Canada. They use the weighted-sum method to optimize the problem, which is to minimize the total travel patient distance and to minimize the total disruption cost to the current system.

Goal Programming is another widely-used method to transform a multi-objective optimization problem to single composite objective. Goals $b_{j}$ are specified for each objective function $\mathrm{F}_{\mathrm{j}}(\mathrm{X})$. Then the total deviation from the goals $\sum_{j=1}^{k}\left|d_{j}\right|$ is minimized, where $d_{j}$ is the deviation from the goal $b_{j}$ for the jth objective.

Blake and Carter (2002) use goal programming to solve the resource allocation problem in hospitals. Their model allows decision makers to set case mix and case volume in hospitals to preserve physician income and minimize disturbance to practice. $\mathrm{Li}$ et al. (2009) use goal programming to solve the multi-objective bed allocation problem among different departments in a hospital, taking account of objectives related to customer service and profits from the hospital manager. Oddoye et al. (2007) use a weighted goal programming method to solve a resource allocation problem in a medical assessment unit. Doctors, nurses and beds are considered as three main resources, and the objectives are to minimize the patients' delay time and the amount of extra resources needed such as doctors' and nurses' time and beds. 
The most commonly-used classical method for the latter case of moving all but one objective to the constraint set is $\epsilon$-Constraint method, which reformulates the multiobjective optimization problem to a single-objective function by keeping just one of the objectives and restricting the rest of the objectives within user-specified values (Deb, 2001). The objective is transformed to:

Minimize $F_{i}(X)$ for some $i$

Subject to $F_{j}(X) \leq \varepsilon_{j}, j=1,2, \ldots, k$, for $j \neq i$

The $\epsilon$-Constraint method depends on the chosen $\epsilon$ vector, which is also supplied by the decision makers.

Mitropoulos et al. (2006) use the constraint method to solve a biobjective optimization problem for locating hospitals and primary healthcare centers. Two objectives are considered: (1) minimization of the distance between patients and facilities, and (2) equitable distribution of the facilities among citizens. They optimize the first objective while the other one is constrained to values that vary through a range of feasible values.

Koyuncu and Erol (2010) develop a multi-objective optimization model to solve the budget allocation problem among preventive vaccination, antiviral treatment and fixed resources to mitigate the impact of pandemic influenza. A case study for Turkey is presented. They use a hierarchical method to solve the multi-objective optimization problem by ordering the objectives in terms of importance based on the preferences of the decision-maker, which is (1) minimizing the number of deaths; (2) minimizing the number of cases; and (3) minimizing the total morbidity days. Then the algorithm uses a multi-level reduced feasible region approach. At the first level, it is attempted to 
minimize the objective function with the highest importance over the feasible region outlined by the system constraints. The value of the objective function at the optimal point is used as a constraint for the next optimization. At level 2, the objective function with second importance is minimized under the new constraint obtained from the first level. This procedure continues in the same way for all objective functions.

The inherent drawback of the above approaches is that prior preference information is required from a decision maker. In practice, it can be very difficult to precisely and accurately identify the preference information (e.g. weights, constraining value), even for someone who is familiar with the problem domain. It is very arbitrary as well. Sometimes even small changes in the preference values (e.g. weights, constraining value) can lead to quite different solutions.

\subsubsection{Determine a Set of Efficient Solutions for Multi-Objective Optimization}

The second general solution approach for multi-objective optimization is to determine an entire Pareto optimal solution set or a representative subset, rather than get a single solution from every single run using the first general approach (as above methods). A Pareto optimal set is a set of solutions that are nondominated by each other (i.e. efficient solutions). While moving from one Pareto solution to another, there is always a certain amount of sacrifice in one objective(s) to achieve a certain amount of gain in the other(s) (Konak et al., 2006). The most widely-used heuristics methods for multi-objective optimization problem are genetic algorithms, simulated annealing, and tabu search. The algorithms of the above heuristics methods were initially developed for the single objective optimization problem, and are tailored to solve multi-objective optimization 
problem. Jones et al. (2002) give a comprehensive overview of the heuristic methods for multi-objective optimization problem.

The concept of genetic algorithm was introduced by (Holland, 1975). Genetic algorithms are inspired by the evolutionist theory explaining the origin of species, which emulate the way species breed and adapt in the field of genetics. Coello (2000) presents a comprehensive survey on generic algorithms based multi-objective optimization techniques. Simulated annealing emulates the way in which a material cools down to its steady state in the field of thermodynamics. Suman and Kumar (2006) present a survey of simulated annealing as a tool to solve multi-objective optimization problem. Tabu search is based on the social concept of 'taboo' in order to provide an effective search technique which avoids local optima (Jones et al., 2002).

Syam and Côté (2010) apply simulated annealing to solve a mixed integer programming model, which is developed for the location and allocation of specified healthcare services in integrated service networks.

Fiedrich et al. (2000) study the assignment of available resources to operational areas to minimize the total number of fatalities during the initial search-and-rescue period after strong earthquakes. They apply both simulated annealing and tabu search methods to solve the assignment problem.

Stummer et al. (2004) use a multi-objective decision support approach to determine the location and size of medical departments in a hospital network. They apply a multiobjective tabu search procedure to identify efficient alternatives and then allow the 
decision makers to interactively explore the corresponding solution space through iterative cluster analysis.

Decision makers often prefer working with Pareto optimal solution set to be given a single solution. A Pareto optimal solution set can be examined for trade-offs. When considering real-life problems, the final solution of the decision-maker always involves making trade-offs. 


\section{CHAPTER 3 SINGLE OBJECTIVE OPTIMIZATION}

\section{MODELS}

This chapter presents five single-objective optimization models for the medium term patient allocation problem encountered by a system of healthcare providers during a pandemic influenza outbreak.

Model 1 is developed to determine the patient allocation over a planning horizon while minimizing the total patient travel distance and satisfying the capacity constraints of various physical healthcare resources. As an extension of the basic Model 1, considerations for desirable resource occupancy rate, patient preference, and the healthcare personnel resources are introduced in Model 2. Desirable resource occupancy rate is used to balance the workload among hospitals. By ensuring that the resource utilization at each hospital does not exceed a predefined desirable occupancy rate, the workload is balanced. Patient preference is used to incorporate the idea that some patients prefer specific healthcare facilities. Finally, Model 2 includes healthcare personnel resources considerations since personnel most likely would become a critical resource due to the high infection rate among healthcare workers. Two techniques are investigated to include the personnel resource capacity in the model. One technique is to include a staff rate parameter which indicates the percentage of physical resources that are staffed. The other technique is to include the specific personnel schedule over the planning 
horizon to determine the personnel capacity. In Model 3, a long planning horizon is divided into several short planning horizons to help shorten the solution run time and reflect capacity changes in different time periods.

When the capacity of a certain resource is exceeded by demand, Models 1,2 and 3 would suggest that there is no feasible solution. However, in reality, even if a certain type of resource is exceeded, the patients who do not require that type of resource can still be accepted by hospitals. The hospitals also would obtain newly released resource capacity when the previously admitted patients are discharged, which could relieve the capacity shortage problem later. Therefore Model 4 allows resource shortages to be estimated and allows patients who do not require the exhausted resource to continue to be allocated to a hospital. Inclusion of a dummy hospital with unlimited resources allows solutions to continue to be generated when one or a few resources are exhausted. This Model 4 can be used to predict when (i.e. which day), where (i.e. which healthcare facility) and what (i.e. which resource) is exceeded by the influenza patients demand during the outbreak. Based on these predictions, decision makers can determine if healthcare facilities need to request additional capacity from the state or national emergency management agencies. If additional resources become available, Model 5 is developed to help optimize the allocation of additional new resources in terms of maximizing the global patient services for the entire healthcare system.

\subsection{Model 1: Basic Model}

As with all mathematical models, a variety of input data or parameters are needed. The models included in this research assume that the in-patient demand or number of patients 
to be admitted to the hospital can be estimated for each population area. This demand data can be obtained from a disease spread model. Disease spread models are used to estimate the infectious disease progression, which helps to prepare for the pandemic influenza outbreak. Various researchers use mathematical or simulation models to estimate the disease spread (Bobashev et al., 2007; Larson, 2007; Naron \& Wasserkrug, 2007; Parker, 2007). Demand estimation is generated over the planning horizon for different patient types requiring different levels of service based on the severity of their illness. Each patient type category requires multiple types of equipment resources (such as ICU beds, non-ICU beds, ventilators) and occupies the resources for a specific time period depending on the length of stay in the hospital. Based on the demand and resource capacity information, Model 1 optimizes the patient allocation with regard to minimizing the patients' cost of access to service as measured here by total distance travelled. The data for demand and resource capacity is assumed to be available in the model. A case study for Metro Louisville, Kentucky is studied in Chapter 4, and the data availability will be discussed in more details in Chapter 4.

The model is described as follows:

Sets:

$\begin{array}{ll}\boldsymbol{H} & \text { hospitals } \\ \boldsymbol{A} & \text { population areas (demand points) } \\ \boldsymbol{T} & \text { periods in the current planning horizon } \\ \boldsymbol{R} & \text { equipment resource types (e.g. non-ICU beds, ICU beds, } \\ & \text { ventilators) }\end{array}$


$\boldsymbol{P}$

$S_{r}$

Parameters:

Distance

Demand $_{p, a, t}$

Capacity $_{r, h, t}$

$\operatorname{LOS}_{p}$ patient types (depending on resources needed and length of stay required by the patients)

set of patient types requiring the service of equipment resource $r \in R$

CONCapacity $_{r, h}$ initial capacity of resource $r$ in hospital $h$ (assume that certain resource quantities are dedicated to pandemic patients at each hospital)

demand of patient type $p$ from area $a$ on day $t$ (in number of patients)

capacity of resource $r$ in hospital $h$ at day $t$

length of stay (i.e. how many days) for patient type $p$

Decision Variables:

$\begin{array}{ll}\boldsymbol{X}_{p, a, h, t} & \text { Number of patient type } p \text { from area } a \text { assigned to hospital } h \\ & \text { on day } t\end{array}$

Objective Function:

$$
\operatorname{Min} \sum_{p} \sum_{a} \sum_{h} \sum_{t} \text { Distance }_{a, h} * X_{p, a, h, t}
$$


Constraints:

$$
\begin{array}{lr}
\text { Capacity }_{r, h, 1}=\text { CONCapacity }_{r, h} & \forall r, h(2) \\
\text { Capacity }_{r, h, t}=\text { Capacity }_{r, h, t-1}-\sum_{a} \sum_{p \in S_{r}} X_{p, a, h, t-1}+\sum_{a} \sum_{p \in S_{r}} X_{p, a, h, t-L O S_{p}} & \forall r, h, t>1(3) \\
\sum_{h} X_{p, a, h, t}=\text { Demand }_{p, a, t} & \forall p, a, t(4) \\
\sum_{a} \sum_{p \in S_{r}} X_{p, a, h, t} \leq \text { Capacity }_{r, h, t} & \forall r, h, t(5) \\
X_{p, a, h, t} \geq 0 &
\end{array}
$$

The objective function of the model (equation 1) minimizes the total travel distance of all patients. Constraints (2) and (3) determine the available capacity of each resource at each hospital on each day. Equation (2) assigns the initial capacity of each resource type in each hospital. It is assumed that certain resource quantities are dedicated to pandemic patients at each hospital. Based on the normal healthcare operation, a regular resource occupancy rate can be observed, then it can be predicted that how many resources at each hospital can be dedicated to pandemic patients. Equation (3) updates the daily resource capacity based on that day's demand and released capacity of patients leaving the hospital. Equation (4) ensures each patient is assigned to a hospital. Equation (5) ensures the capacity of each equipment resource at each hospital is not exceeded. Equation (6) are the nonnegativity constraints.

Model 1 was tested using problems of various sizes to compare the respective solution runtimes. Since the purpose of running different problem sizes is to test the run time, 
randomly generated data, rather than real-world data, was used. The size of the model depends on the number of hospitals, the number of population areas, the number of periods in the planning horizon, the number of resource types, and the number of patient types. The model was solved using LINGO 11.0 on WINDOWS XP of a Pentium 4 PC with 1 GB memory. The run times for the various cases are given in Table 1.

Table 1. Model 1 runtime for various data set sizes

\begin{tabular}{|c|c|c|c|c|}
\hline & Case1 & Case2 & Case3 & Case4 \\
\hline Number of Hospitals & 8 & 16 & 16 & 16 \\
\hline Number of Areas & 8 & 20 & 30 & 40 \\
\hline Number of Days & 8 & 10 & 15 & 30 \\
\hline Number of Resource Types & 8 & 8 & 8 & 8 \\
\hline Number of Patient Types & 8 & 8 & 8 & 8 \\
\hline Number of Decision Variables & 5056 & 28032 & 61312 & 161152 \\
\hline Number of Constraints & 2497 & 6593 & 11153 & 24833 \\
\hline Runtime(hh:mm:ss) & $00: 00: 23$ & $00: 05: 21$ & $00: 20: 42$ & $01: 43: 33$ \\
\hline
\end{tabular}

As can be seen in Table 1, as the data sets get larger from case 1 to case 4 , the number of decision variables and the number of constraints increase dramatically and the model run time increases accordingly. Therefore, for larger instances of the model the run time is a concern. While longer run times are not the utmost concern for medium term planning, shorter run times are advantageous for performing what-if analysis. Dividing the long planning horizon into several short planning horizons is a way to reduce the run time, which is implemented in Model 3.

\subsection{Model 2: Additional Characteristics}


To make Model 1 more accurately reflect real world situations, additional parameters and constraints are included in the basic Model 1. Desirable occupancy rate, patient preference, and healthcare personnel capacity are introduced in Model 2.

Desirable occupancy rate is a way to balance the workload among hospitals, by ensuring that the resource utilization at each hospital does not exceed a predefined desirable occupancy rate. The occupancy rate can be determined by the decision maker and different levels can be applied to see the impact on the performance. Such a parameter and the corresponding constraints can be used to avoid the situation where some healthcare sites are overloaded while others are underutilized. CONCapacity $r, h$ indicates the resource capacity to be dedicated to the pandemic influenza. The parameter OccupancyRate is included to indicate the predefined desirable percentage of resource capacity to be occupied (i.e. the range is $[0,1])$. The user is allowed to define the occupancy rate. The predefined desirable resource occupancy rate can be changing during the development of pandemic. At early stage of the development, the hospitals may not be very stressed by the patients, a relatively low occupancy rate (e.g. $60 \%$ to $80 \%$ ) can be applied to spread out the patients among hospitals. As the development of the disease, the hospitals can get much more overwhelmed by the surge demand, then a higher occupancy rate (e.g. $80 \%$ to $100 \%$ ) must be applied. In addition, what-if analysis can also be applied to evaluate the impact of different levels of occupancy rate on the service to patients. The following constraints (7) are included to ensure that the resource utilization does not go beyond the predefined desirable occupancy rate.

$$
\text { Capacity }_{r, h, t}-\sum_{a} \sum_{p \in S_{r}} X_{p, a, h, t} \geq(1-\text { OccupancyRate })^{*} \text { CONCapacity }_{r, h} \quad \forall r, h, t(7)
$$


Considerations for patient preference are also included in Model 2. From a patient's viewpoint, quality of care and access to services are key concerns. In the initial model, it is assumed that the quality of care among hospitals is the same, and the travel cost is the only concern for the patients. However, in reality, some patients prefer going to a certain hospital for various reasons, such as 1) they need a special service in a certain hospital (e.g. children go to children's hospital), 2) insurance concerns, 3) consideration of hospital's reputation, etc. It is assumed that a certain percentage of a certain patient type prefer a certain hospital. Therefore, additional parameters PatientPreference $p_{p, h}$ are included in the model to indicate the percentage of patient type $p$ who prefer (or have to) go to hospital $h$. PatientPreference ${ }_{p, h}$ is a rate, then the range is $[0,1]$. The following constraints are introduced in Model 2:

$$
X_{p, a, h, t} \geq \text { PatientPreference }_{p, h} * \text { Demand }_{p, a, t}
$$

During a pandemic influenza outbreak, the healthcare personnel are among the high-risk group to be infected. The infection of healthcare personnel would limit the number of patients who can be treated. A shortage of healthcare personnel can also affect the usage of other physical resources. Therefore, it is necessary to consider the healthcare personnel capacity to determine the patient allocation. In the basic Model 1, only physical resource capacity is included. To corporate the healthcare personnel capacity in the model, two possible techniques are considered: 1) staffed rate is applied to indicate the percentage of physical resources that are staffed; 2) healthcare personnel schedule is used to include the specific personnel capacity level. The estimation of the staffed rate or personnel schedule can be obtained based on the decision makers' expertise. Experiments using different 
levels of the staffing rate or personnel schedule can be investigated as well to reflect different situations.

When staffed rate is used to reflect the capacity related to healthcare personnel, the parameter StaffRate $e_{r, t}$ is introduced in the model, indicating the staffed rate for physical equipment resource $r$ in hospital $h$ on day $t$, then constraints (2) and (3) related to capacity in Model 1 are modified as follows:

Capacity $_{r, h, 1}=$ CONCapacity $_{r, h}{ }^{*}$ StaffRate $_{r, h, 1} \quad \forall r, h(9)$

Capacity $_{r, h, t}=$ StaffRate $_{r, h, t} *\left(\right.$ Capacity $\left._{r, h, t-1}-\sum_{a} \sum_{p \in S_{r}} X_{p, a, h, t-1}+\sum_{a} \sum_{p \in S_{r}} X_{p, a, h, t-\operatorname{LoS}_{p}}\right)$

When a specific healthcare personnel schedule is used, additional sets, parameters and intermediate variables (i.e. they are not main decision variables, but their values are computed in the models) need to be included in the model as follows:

Sets:

$S \quad$ Healthcare personnel (staff) resource types (e.g. doctor, nurse, and lab technician)

$S_{s} \quad$ Set of patient types requiring the service of personnel resource $s \in S$

Parameters: 
StaffHrs $_{p, s} \quad$ number of staff hours needed by patient type $p$ for personnel resource $s$ each day

StaffCap $_{s, h, t} \quad$ staff capacity (man-hours) for personnel resource $s$ at hospital $h$ on day $t$

Intermediate Variables:

NoPatients $s_{p, h, t} \quad$ number of patient type $p$ in hospital $h$ on day $t$

The predicted number of patients at each hospital is calculated for each day and should not exceed the personnel capacity constraints. The capacity constraints related to personnel resources are added as follows:

$$
\begin{array}{lr}
\text { NoPatients }_{p, h, 1}=\sum_{a} X_{p, a, h, 1} & \forall p, h(11) \\
\text { NoPatients }_{p, h, t}=\text { NoPatients }_{p, h, t-1}+\sum_{a} X_{p, a, h, t}-\sum_{a} X_{p, a, h, t-\text { LOS }_{p}} & \forall p, h, t>1(12) \\
\sum_{p \in S_{s}} \text { NoPatients }_{p, h . t} * \text { StaffHrs }_{p, s} \leq \text { StaffCap }_{s, h, t} & \forall s, h, t(13)
\end{array}
$$

\subsection{Model 3: Model for Multiple Planning Horizons}

In models 1 and 2, a constant starting condition is used for each resource's capacity constraint and then the daily capacity is updated based on the newly admitted patients and the discharged patients. 
In model 3, a long planning period, such as a 2-month wave for the pandemic outbreak, is divided into several shorter planning periods (e.g. consecutive weekly plans can be made during the outbreak). Then the capacity is updated based on the new patients, the discharged patients and the new released capacity from the last planning period.

Dividing a long planning horizon into several short planning horizons has the following advantages:

- Reduces the solution run times. The shorter planning horizon, the less run time required to find a solution, as demonstrated in Table 1. It should be noted that shortening the planning horizon and solving the model iteratively does not guarantee the overall, global optimal solution. However, the suboptimal solution found by dividing the time horizon and 'adding up' the short optimal solutions is acceptable for the decision makers to determine patient allocation solutions for a pandemic influenza outbreak.

- Offers the flexibility to update the input to the models during the long planning horizon. If the model is solved once for a long planning horizon, an initial starting capacity condition is set and may be difficult to update during a long planning horizon. If several short planning horizons are considered, the resource capacity condition can be updated to reflect the additional capacity that may be obtained during the surge demand period. When the capacity is exceeded by the demand, extra capacity can be added in the system, and the problem can be solved based on the new capacity constraints.

- Obtains feasible solutions before resources run out in a long planning horizon. If the model is solved once for a long planning horizon, no feasible solution would 
be obtained if resources run out at certain point during the pandemic influenza outbreak, which has a high possibility. In this case, if the model is solved in short planning horizons, a feasible solution would be obtained for the periods when the capacity is not exceeded by the demand.

To connect the consecutive short planning horizon models with one another, the output from the previous planning horizon is used as the starting condition for the current planning horizon as follows:

- The final capacity from the previous planning horizon is fed into the current planning period as the starting capacity, or the current real capacity situation can be input as a starting condition to compute the current planning horizon, which would give more reliable results. Also, the additional surge capacity can be added to the system after several short planning horizons.

- The current planning horizon would carry the newly released capacity from the patients who were admitted in the previous planning horizon and are discharged in this planning horizon. The capacity released for the following planning horizon is calculated in the current planning horizon as well.

Sets:

$\boldsymbol{H}$ hospitals

$\boldsymbol{A}$ population areas (demand points)

T periods in the current planning horizon

$T$ periods in the next planning horizon

$\boldsymbol{R}$ equipment resource types (e.g. non-ICU beds, ICU beds, ventilators) 
$S$ personnel (staff) resource types (e.g. doctors, nurses, lab technician)

$\boldsymbol{P}$ patient types (depending on resources needed and length of stay required by the patients)

$S_{r} \quad$ set of patient types requiring the service of equipment resource $r \in R$

$S_{s} \quad$ set of patient types requiring the service of personnel resource $s \in S$

Parameters:

Distance $_{a, h} \quad$ distance from population area $a$ to hospital $h$

Demand $_{p, a, t} \quad$ demand of patient type $p$ from area $a$ on day $t$ (in number of patients)

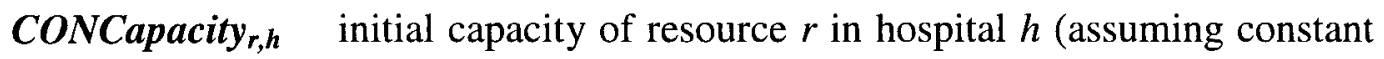
capacity allocated to pandemic patients)

StaffHrs $_{p, s} \quad$ staff hours needed for patient type $p$ of staff resource $s$

$\operatorname{LOS}_{p} \quad$ length of stay (how many days) for patient type $p$

StaffCap $_{s, h, t} \quad$ staff capacity (man-hours) for personnel resource $s$ at hospital $h$ on day $t$

InitPatients $_{p, \boldsymbol{h}} \quad$ number of patient type $p$ at hospital $h$ prior to the planning horizon

Intermediate Variables:

NoPatients $s_{p, t}$

number of patient type $p$ at hospital $h$ on day $t$

ReleasedPatients $_{p, h, t} \quad$ number of patient type $p$ admitted from the previous planning horizon who will be released at hospital $h$ on day $t$ of the current planning horizon 
NextReleasedPatients ${ }_{p, h, t}$, number of patient type $p$ admitted from the current planning horizon who will be released at hospital $h$ on day $t^{\prime}$ of the next planning horizon

Decision Variables:

$X_{p, a, h, t} \quad$ number of patient type $p$ from area $a$ assigned to hospital $h$ on day $t$

Objective Functions:

$$
\operatorname{Min} \sum_{p} \sum_{a} \sum_{h} \sum_{t} X_{p, a, h, t} * \text { Distance }_{a, h}
$$

Constraints:

$$
\begin{array}{ll}
\sum_{h} X_{p, a, h, t}=\text { Demand }_{p, a, t} & \forall p, a, t(15) \\
\text { NoPatients }_{p, h, 1}=\text { InitPatients }_{p, h}+\sum_{a} X_{p, a, h, 1}-\text { ReleasedPatients }_{p, h, 1} & \forall p, h(16) \\
\text { NoPatients }_{p, h, t}=\text { NoPatients }_{p, h, t-1}+\sum_{a} X_{p, a, h, t} & \\
-\sum_{a}\left(X_{p, a, h, t-\text { LOS }_{p}}\right)_{- \text {ReleasedPatients }_{p, h, t}} & \forall p, h, t>1(17) \\
\text { NextReleasedPatients }_{p, h, t^{\prime}}=\sum_{a} X_{p, a, h, t^{\prime}+T-\text { Los }_{p}} & \forall p, h, t^{\prime}(18) \\
\sum_{p \in S_{r} \text { NoPatients }_{p, h, t} \leq \text { ConCapacity }_{r, h}} & \forall r, h, t(19) \\
\sum_{p \in S_{s}}\left(\text { NoPatients }_{p, h, t} * \text { StaffHrs }_{p, s}\right) \leq \text { StaffCap }_{s, h, t} &
\end{array}
$$




$$
X_{p, a, h, t} \geq 0
$$

Constraints (15) define the demand constraint. Every patient is assigned to a specific healthcare facility. Constraints (16) through (20) define the capacity constraint for each resource type at each facility. Constraints (16) and (17) calculate the number of patients of each type in each hospital each day. These constraints calculate the number of patients as: number of patient type $p$ on day $t=$ number of patient type $p$ on day $(t-1)+$ newly admitted patient type $p$ on day $t$ - released patient type $p$ on day $t$ who are admitted at current planning horizon - released patient type $p$ on day $t$ who are admitted from previous planning horizon. It is assumed that the length of stay $(L O S)$ for a patient type is not greater than the planning horizon. The number of released patients is calculated by considering only the previous and current planning horizon. Constraints (18) calculate the number of each type of patient in each hospital admitted in the current planning horizon and released in the next planning horizon. Therefore, NextReleasedPatient $s_{p, h, t^{\prime}}$ calculated in the current planning horizon will become ReleasedPatient $s_{p, h, t}$ for the next planning horizon. Constraints (19) and (20) are the capacity constraints to make sure both the equipment and personnel capacity is not exceeded by the demand. Constraints (21) are the nonnegativity constraints.

\subsection{Model 4: Model for Multiple Planning Horizons and Allowed}

\section{Resource Shortages}

During a pandemic outbreak, a wave of patient demand is expected which will peak after a certain time period and then start to decrease. If the patient demand for a specific resource exceeds the capacity, the previous three models will not give a feasible solution 
due to the capacity constraints being violated. However, in reality, even when one type of resource is exceeded, those patients who do not require that specific resource can still be accepted by hospitals. The hospitals will also obtain newly released capacity when previously admitted patients are discharged, which could relieve the capacity shortage problem later in the planning horizon. Therefore it is desirable to develop a patient allocation model that can be used for planning over the entire horizon, even if some resources are temporarily depleted. In Model 4, a dummy hospital with unlimited resources is introduced to allow feasible solutions for the entire planning horizon. The other improvement of Model 4 is that the shortage of a resource is calculated to indicate the reason some patients are rejected by regular healthcare facilities, i.e. admitted to the dummy hospital. Then the decision maker can be alerted about the resource shortage and corresponding additional quantities of resources can be requested from emergency management agency.

A dummy hospital is introduced in the model which is used to absorb the patients who cannot be assigned to a real hospital when there is a capacity shortage. Patients are only allocated to the dummy hospital when all other hospitals are experiencing a capacity shortage. Therefore the patients who are assigned to the dummy hospital can be considered as the rejected patients in the real situation. The big- $M$ method is used in the model (big- $M$ stands for a very large number). The dummy hospital is assumed to have $M$ capacity for each resource to make sure that the dummy hospital can accept all the exceeded demand, and the distance between the dummy hospital and each population area is $M$. The objective function is: 


$$
\text { Min } \sum_{p} \sum_{a} \sum_{h} \sum_{t} \text { Distance }_{a, h} * X_{p, a, h, t}+\sum_{p} \sum_{a} \sum_{t} \text { Distance }_{a, h^{\prime}} * X_{p, a, h^{\prime}, t} *(T+1-t)
$$

The first term of the objective function is the same as the previous model, which is to minimize the total travel distance between all patients and their assigned hospital. The second term ( $h^{\prime}$ is the dummy hospital) is to minimize the number of the rejected patients and to make sure that the patients are assigned to the dummy hospital only when the other hospitals cannot accept the patients. The distance between the dummy hospital and each population area is large enough to make sure that the number of the patients who are assigned to the dummy hospital is minimized, which means that only the exceeded demand is assigned to the dummy hospital. In the Model 4 objective function, the distance is multiplied by $(T+1-t)$, to make sure that patients are accepted to the dummy hospital only when the real hospitals cannot accept patients due to a capacity shortage. If the second term is written as $\sum_{p} \sum_{a} \sum_{t}$ Distance $_{a, h^{\prime}} * X_{p, a, h, t}$, then the number of patients who are assigned to the dummy hospital can be minimized, however it could happen that the patients are rejected (assigned to the dummy hospital) before the capacity of the other hospitals is exceeded. Therefore the term $(T+l-t)$ is used to make sure that the assignment to the dummy hospital only occurs when the other, real, hospitals cannot accept the patient. $T$ is the total periods in the planning horizon, and $t$ is the period index, therefore $T+1-t>0 . T+1-t$ is expected to be as small as possible to minimize the second term in the objective function, so $t$ is as large as possible, which means that the assignment to the dummy hospital is as late as possible, so only when the other, real, hospitals cannot accept the patients, the dummy hospital would start to accept the patients. 
In addition, the other improvement of Model 4 is to calculate the resource shortage in the system to indicate why some patients are admitted to the dummy hospital, meaning rejected by regular healthcare sites. The following additional parameters are included in Model 4:

ResourceShortage $_{r, t} \quad$ shortage of physical resource $r$ on day $t$

StaffHrSShortage $_{s, t} \quad$ Shortage of staff hours on day $t$

The resource shortage is calculated by the difference between resource needed by the demand and the resource held by the healthcare facilities. The needed resource is calculated based on all patients including the ones admitted to dummy hospital, while the held resource is calculated based on capacity at all real healthcare sites, as follows:

$$
\begin{aligned}
\text { ResourceShortage }_{r, t}= & \sum_{h} \text { CONCapacity }_{r, h}- \\
& \sum_{h} \sum_{p \in S_{r}} \text { NoPatients }_{p, h, t}-\sum_{p \in S_{r}} \text { NoPatients }_{p, h h^{\prime},}
\end{aligned}
$$

$$
\begin{gathered}
\text { StaffHrsShortage }_{s, t}=\text { StaffCap }_{s, t}-\sum_{h} \sum_{p \in S_{s}} \text { NoPatients }_{p, h, t} * \text { StaffHrs }_{p, s}- \\
\sum_{p \in S_{s}} \text { NoPatients }_{p, h^{\prime}, t} * \text { StaffHrs }_{p, s}
\end{gathered}
$$

Overall, Model 4 allows solutions to be obtained for the planning horizon even when a resource's capacity is exceeded. The advantages include:

- For the patients who do not need the shortage resource, the model is able to assign them to appropriate hospitals; 
- At some time one or more resources may be depleted. Later in the planning horizon, the depleted resources can regain capacity when patients are discharged thereby allowing new patients to claim the resource.

- The model can indicate when resources would be depleted.

- The model calculates the resource shortage to indicate the magnitude of the resource shortage.

\subsection{Model 5: Model Considering the Allocation of Additional Resources}

Model 4 can be used to predict when (i.e. which day), and which resource(s) is exceeded by the influenza patients demand during the outbreak. Based on these predictions, decision makers can determine if healthcare facilities need to increase medical capacity (e.g. put the patients at the hallway), or request additional resource capacity from the state or national stockpile. These stockpiles supplement and re-supply healthcare facilities and state/local public health agencies in the event of an emergency. If the healthcare facilities could receive additional resources, Model 5 is proposed to optimize the allocation of additional resources in terms of maximizing the patient services. The additional parameters and decision variables, compared to above models, are as follows:

Parameters:

AddRes $_{r} \quad$ number of additional equipment resource $r$

AddPRes $_{s} \quad$ man-hours of additional personnel resource $s$

Decision Variables: 
AddResAllc $c_{r, h} \quad$ number of resource $r$ allocated to hospital $h$

AddPResAll $c_{s, h} \quad$ man-hours of personnel resource $s$ allocated to hospital $h$

Constraints:

$$
\begin{aligned}
& \sum_{h} \text { AddResAllc }_{r, h} \leq \text { AddRes }_{r} \\
& \sum_{h} \text { AddPResAllc }_{s, h} \leq \text { AddPRes }_{s}
\end{aligned}
$$

Constraints (25) and (26) are the additional resource allocation constraints to make sure the allocation does not exceed the available additional resources including both equipment resources and personnel resources.

$$
\begin{array}{ll}
\sum_{p \in S_{r}} \text { NoPatients }_{p, h, t} \leq \text { ConCapacity }_{r, h}+\text { AddResAllc }_{r, h} & \forall r, h, t(27) \\
\sum_{p \in S_{s}}\left(\text { NoPatients }_{p, h, t} * \text { StaffHrs }_{p, s}\right) \leq \text { StaffCap }_{s, h, t}+\text { AddPResAll }_{s, h} & \forall s, h, t(28)
\end{array}
$$

Constraints (27) and (28) are the updated capacity constraints which consider the newly allocated additional resources including both equipment and personnel resources.

Model 5 is applied in a case study from Metro Louisville, Kentucky in Chapter 4 to demonstrate how the model can help decision makers determine the patient allocation and potential resource shortages in the healthcare in a healthcare network during an influenza pandemic outbreak, as well as help to decide the potential surge medical resource allocation. 


\section{CHAPTER 4 CASE STUDY AND SENSITIVITY ANALYSIS}

The models presented in Chapter 3 can be used for patient allocation during a pandemic influenza outbreak. Moreover the models can be used to predict a resource shortage during an outbreak. Based on these predictions, decision makers can determine if healthcare facilities need to increase medical capacity (e.g. put the patients at the hallway), or request additional capacity from the state or national emergency management agents. If the healthcare facilities could receive new resources, the models help to optimize the allocation of additional new resources in terms of maximizing the patient services.

In this Chapter, numerical results for a case study from Metro Louisville, Kentucky are presented to demonstrate how the model can help decision makers (e.g. Kentucky hospital association, Kentucky Emergency Operations Center) determine the patient allocation and potential resource shortages in the healthcare system, as well as help to decide the potential surge medical resource allocation. In addition, sensitivity analysis is applied to the mathematical models, which is mainly used to help decide how different quantities of additional resources would improve the service to the patients. Considering the high demand during a pandemic influenza outbreak, a resource shortage is most likely to happen. Therefore, an important task for the decision makers to decide is how much additional resource should be requested and how different quantities of resources would impact the service to the patients. Finally, a web-based application based on the models 
presented in Chapter 3 was developed by research colleagues and is included here as an example of the real world application of this work.

\subsection{Case Study}

A case study is presented here to demonstrate how the models developed in Chapter 3 can help decision makers determine the patient allocation and potential resource shortages in the healthcare system, as well as help to decide the potential surge medical resource allocation.

\subsubsection{Model Input Data}

A case study from Louisville-Jefferson County, Kentucky is studied. In 2009 LouisvilleJefferson County, Kentucky was estimated to have a population of approximately 722,000 people and cover 400 square miles. For this case study, Louisville-Jefferson County was divided into 16 areas as shown in Figure 1. There are 170 census tracts in Louisville-Jefferson County. The influenza patient demand from each census tract is too trivial since the population size from each census tract is small. Hence, 170 census tracts are combined into 16 areas, then the patient demand from each area is more substantial and it is easier to implement the patient allocation decision while considering relatively smaller number of areas. Seven hospitals were considered available to receive patients during an influenza pandemic; the location and numbering scheme of these hospitals is shown in Figure 1. The distance (in miles) between the geographic centroid of each population area and each hospital is shown in Table 2. 


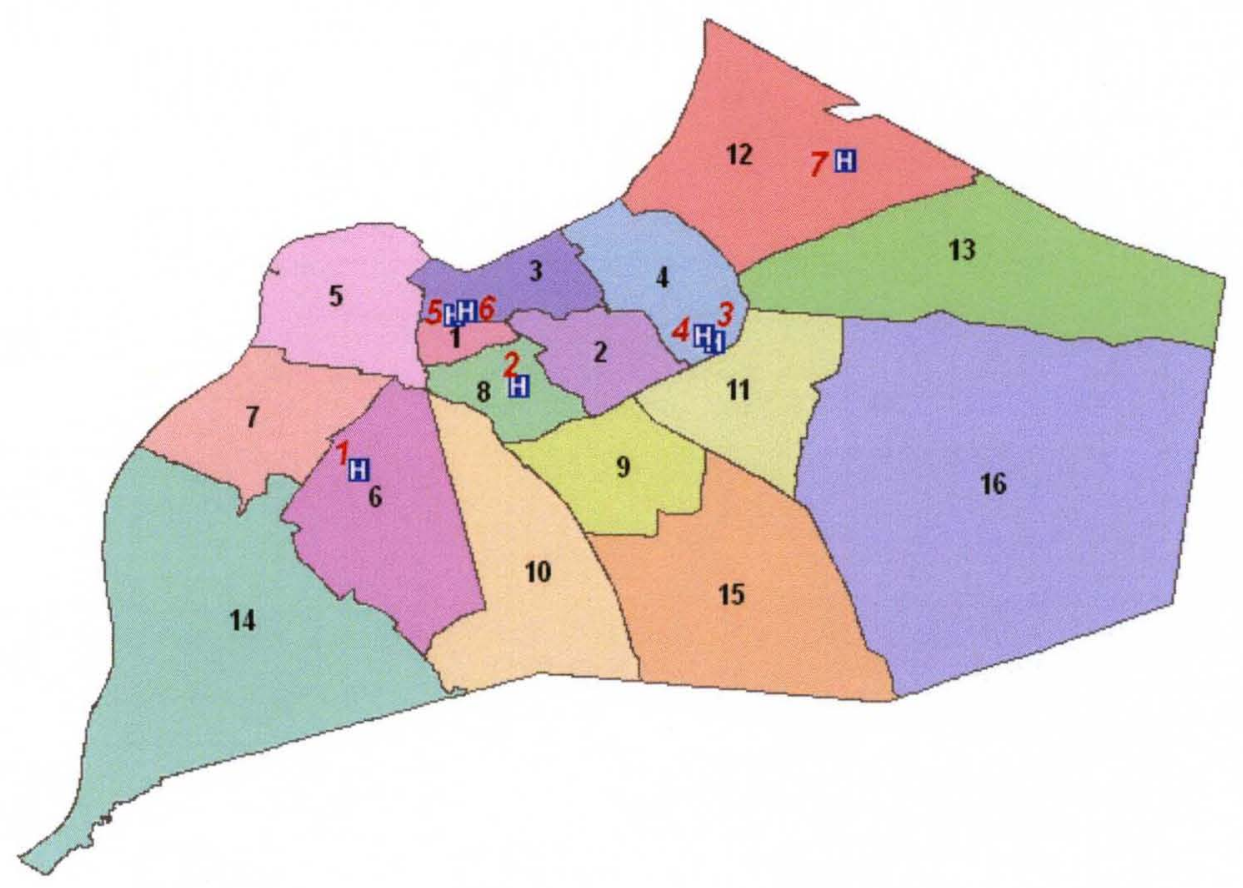

Figure 1. Map of Metro Louisville, Kentucky

Table 2. Distance (in miles) between population areas and hospitals

\begin{tabular}{|c|c|c|c|c|c|c|c|}
\hline & Hospital 1 & Hospital 2 & Hospital 3 & Hospital 4 & Hospital 5 & Hospital 6 & Hospital 7 \\
\hline Area 1 & 4.64 & 2.06 & 6.04 & 5.83 & 0.85 & 0.86 & 10.73 \\
\hline Area 2 & 6.75 & 2.20 & 2.74 & 2.60 & 3.64 & 3.25 & 8.36 \\
\hline Area 3 & 6.59 & 2.98 & 4.99 & 4.67 & 1.56 & 1.22 & 8.86 \\
\hline Area 4 & 9.49 & 4.98 & 2.32 & 1.88 & 5.23 & 4.84 & 5.51 \\
\hline Area 5 & 5.04 & 4.79 & 8.74 & 8.48 & 2.51 & 2.92 & 12.61 \\
\hline Area 6 & 1.71 & 4.98 & 9.33 & 9.38 & 6.47 & 6.54 & 15.41 \\
\hline Area 7 & 2.47 & 5.99 & 10.81 & 10.70 & 5.55 & 5.86 & 15.90 \\
\hline Area 8 & 4.11 & 0.45 & 5.27 & 5.20 & 2.78 & 2.61 & 10.92 \\
\hline Area 9 & 6.25 & 3.35 & 4.32 & 4.56 & 6.10 & 5.82 & 10.79 \\
\hline Area10 & 4.63 & 5.42 & 8.38 & 8.58 & 7.85 & 7.76 & 14.83 \\
\hline Area11 & 9.79 & 5.66 & 1.78 & 2.22 & 7.58 & 7.18 & 7.42 \\
\hline Area12 & 13.75 & 9.31 & 5.75 & 5.45 & 9.00 & 8.67 & 1.55 \\
\hline Area13 & 16.23 & 11.79 & 6.96 & 7.06 & 12.82 & 12.41 & 4.88 \\
\hline Area14 & 5.89 & 10.16 & 14.52 & 14.59 & 11.20 & 11.37 & 20.63 \\
\hline Area15 & 9.81 & 8.13 & 7.64 & 8.04 & 10.90 & 10.63 & 13.52 \\
\hline Area16 & 15.26 & 11.81 & 8.11 & 8.50 & 14.02 & 13.63 & 10.74 \\
\hline
\end{tabular}


FluSurge 2.0, developed by CDC, (Zhang et al., 2006) was used to generate the demand estimation of influenza patients used in this case study. FluSurge users are asked to provide estimates of their local population in three age groups ( 0 to 19,20 to 64 , and $65+$ years) that can be obtained from census data, and are able to select the duration of a pandemic $(6,8$, or 12 weeks) and the gross clinical attack rate $(15 \%, 25 \%$, or $35 \%)$, i.e. the percentage of population with a symptomatic case of influenza. Based on these data, FluSurge estimates the number of hospital admissions each day due to pandemic influenza for three scenarios (most like scenario, minimum scenario, and maximum scenario). FluSurge estimates the total hospitalizations for the entire user-defined pandemic duration based on the gross attack rate and distributes the total number of hospitalizations over each week. A triangular distribution is assumed for weekly distribution of hospital admissions with fewer influenza hospitalizations occurring at the beginning and end of the duration and a peak occurring in the middle of the pandemic. During the weeks before the peak of the pandemic, FluSurge assumes a 3\% daily increase in hospitalizations due to influenza. After the pandemic peak, a 3\% daily decrease in patients is assumed by FluSurge.

The hospital admissions of three types of patients are estimated by FluSurge; Type 1 patients are moderate severity patients who occupy a non-ICU bed and stay in a hospital for 5 days, Type 2 patients are severe patients who occupy an ICU bed and stay in a hospital for 10 days, and Type 3 patients are more severe patients who occupy both an ICU bed and a ventilator and stay in a hospital for 10 days. This case study uses the FluSurge default assumption of $85 \%$ of influenza hospitalizations are for Type 1 patients, 
$7.5 \%$ of hospitalizations are Type 2 patients and the remaining $7.5 \%$ of hospitalizations are Type 3 patients.

Based on the three types of pandemic patients considered in FluSurge, Non-ICU beds, ICU beds and ventilators are the three types of critical equipment resources to be considered in this case study. The total amount of equipment resources in the seven Metro Louisville hospitals in this case study are obtained from a statewide-used software, WebEOC (2010), which is a web-enabled crisis information management system used at the Kentucky Emergency Operations Center for natural disaster response and recovery efforts. Each of the seven hospitals considered in this case study have equipment resource capacities ranging from 100 to 200 non-ICU beds and 20 to 200 ICU beds. The total amount of each type resource at each hospital is shown as in Table 3:

Table 3. Total number of each type resource at hospitals

\begin{tabular}{|c|c|c|c|c|c|c|c|}
\hline & Hospital1 & Hospital2 & Hospital3 & Hospital4 & Hospital5 & Hospital6 & Hospital7 \\
\hline Non-ICU & 104 & 153 & 139 & 204 & 103 & 168 & 111 \\
\hline ICU Bed & 81 & 88 & 72 & 206 & 172 & 56 & 16 \\
\hline Ventilator & 4 & 11 & 3 & 20 & 7 & 12 & 7 \\
\hline
\end{tabular}

\subsubsection{Numerical Results}

Based on the expertise of the Louisville emergency response personnel consulted for this research, such as emergency preparedness/trauma coordinator from Kentucky Hospital Association, Kentucky Strategic National Stockpile Coordinator, physicians from local hospitals, Coordinator from Louisville Metro Emergency Management Agency, and Consultant from Louisville Metro Emergency Management, this case study investigates the patient allocation and resource allocation during a estimated pandemic influenza 
outbreak with $35 \%$ gross clinical attack rate and 6-week duration for the most likely scenario.

Only physical equipment resources are considered in this case study since accurate personnel scheduling data is not currently available for these hospitals; however, it is believed that in a pandemic scenario the hospitals would have a more precisely-tracked scheduling system and personnel resources would become critical, so the personnel constraints are included in the models.

It is assumed that a certain percentage of hospital resources would be dedicated to the pandemic influenza patients. Considering that the average bed occupancy rate in hospitals is $80 \%$ to $90 \%$ during normal, non-influenza operations and assuming that less than normal occupancy of non-influenza patients would be expected during a pandemic situation, $25 \%$ of the total resource capacity in hospitals was selected as a reasonable level to be dedicated to pandemic in-patients in a starting experiment in this case study.

In this case study, the model with multiple planning horizons and allowable resource shortage (i.e. Model 5 in Chapter 3) is applied. As stated above, the hospitalization for a 6-week pandemic outbreak is estimated by FluSurge 2.0. The detailed demand estimation is shown in the Appendix. The model is solved for every two weeks for the planning horizon. The mathematical model was solved in seconds using LINGO 11.0 on a Pentium 4 PC with 1 GB memory. To explain how the model can help suggest the patient allocation, the allocation results for each type of patient for the first two-week planning horizon are summarized in Table 4, Table 5, and Table 6. It shows the summary of to which hospital each type of patient from each area should be allocated (the hospital 0 
indicates the dummy hospital in the table). For example, on day 5 influenza Type 1 patients from area 5 should be admitted to hospitals 5 and 6 . As stated earlier, it is assumed these Type 1 patients will occupy a non-ICU bed for 5 days, Type 2 patients will occupy an ICU bed for 10 days, and Type 3 patients will occupy an ICU bed and a ventilator for 10 days. Table 4, Table 5 , and Table 6 provide a summary of the model results; detailed model results would include the number of patients to allocate to each hospital each day, as shown in the Appendix.

\section{Table 4. Patient type 1 allocation summary}

\begin{tabular}{|c|c|c|c|c|c|c|c|c|c|c|c|c|c|c|}
\hline & D1 & D2 & D3 & D4 & D5 & D6 & D7 & D8 & D9 & D10 & D11 & D12 & D13 & D14 \\
\hline A1 & 6 & 6 & 6 & 6 & 6 & 6 & 6 & 6 & 6 & 6 & 6 & 6 & 6 & 6 \\
\hline A2 & 4 & 4 & 4 & 4 & 4 & 4 & 4 & 4 & 4 & 4 & 4 & 4 & 4 & 4 \\
\hline A3 & 6 & 6 & 6 & 6 & 6 & 6 & 6 & 6 & 6 & 6 & 6 & 6 & 6 & 6 \\
\hline A4 & 4 & 4 & 4 & 4 & 4 & 4 & 4 & 4 & 4 & 4 & 4 & 4 & 4 & 4 \\
\hline A5 & 5 & 5 & 5 & 5 & 5,6 & 5 & 5 & 5 & 5 & 5,6 & 5 & 5 & 5 & 5 \\
\hline A6 & 1,2 & 1,2 & 2 & 1,6 & 2,6 & $1,2,6$ & $1,2,6$ & 1,2, & 1 & 2,6 & 1,2 & 1 & 1,2 & 1,2 \\
\hline A7 & 5 & 5 & 5 & 5 & 6 & 5,6 & 5,6 & 5,6 & $\begin{array}{c}5 \\
6\end{array}$ & 6 & 6 & 6 & 6,0 & 6,0 \\
\hline A8 & 2 & 2 & 2 & 2 & 2 & 2 & 2 & 2 & 2 & 2 & 2 & 2 & 2 & 2 \\
\hline A9 & 2 & 2 & 2,4 & 2 & 4 & 2,4 & 2 & 2 & $\begin{array}{c}2, \\
4\end{array}$ & 4 & 2,3 & 2,3 & 2,3, & 2,4 \\
\hline A10 & 2 & 2 & 2 & 2 & 2 & 2 & 2 & 2 & 2 & 2 & 2 & 2 & 2 & 0 \\
\hline A11 & 3 & 3 & 3 & 3 & 3,4 & 3 & 3 & 3 & 3 & 3,4 & 3 & 3 & 3 & 3 \\
\hline A12 & 7 & 7 & 7 & 7 & 7 & 7 & 7 & 7 & 7 & 7 & 7 & 7 & 7 & 7 \\
\hline A13 & 7 & 7 & 7 & 7 & 4,7 & 7 & 7 & 4,7 & 4, & 4 & 3,4, & 3,7 & 7,0 & 7,0 \\
\hline A14 & 1 & 1 & 1 & 1 & 6 & 1 & 1 & 0 & 0 & 0 & 0 & 0 & 0 & 0 \\
\hline A15 & 3 & 3 & 3 & 3,4 & 4 & 3 & 3 & 3,0 & $\begin{array}{c}3, \\
0\end{array}$ & 0 & 3,0 & 3,0 & 0 & 0 \\
\hline A16 & 3 & 3 & 4 & 4 & 4 & 3,4 & 3,4 & 0 & 0 & 0 & 0 & 0 & 0 & 0 \\
\hline
\end{tabular}

During the first two weeks of the pandemic development, some areas would not have the hospitalization cases for patient types 2 and 3. Therefore Table 5 and Table 6 for the patient allocation summary only show the areas which have the hospitalization cases; the empty cells also indicate no hospitalization cases early in the planning horizon. 
Table 5. Patient type 2 allocation summary

\begin{tabular}{|c|c|c|c|c|c|c|c|c|c|c|c|c|c|c|}
\hline & Day1 & D2 & D3 & D4 & D5 & D6 & D7 & D8 & D9 & D10 & D11 & D12 & D13 & D14 \\
\hline Area5 & & & & & & & & & & & & 5 & 5 & 5 \\
\hline Area6 & & & & & 1 & 1 & 1 & 1 & 1 & 1 & 1 & 1 & 1 & 1 \\
\hline Area11 & & & & & & & & & & & 3 & 3 & 3 & 3 \\
\hline Area14 & & & & & & & & 1 & 1 & 1 & 1 & 1 & 1 & 1 \\
\hline
\end{tabular}

Table 6. Patient type 3 allocation summary

\begin{tabular}{|c|c|c|c|c|c|c|c|c|c|c|c|c|c|c|}
\hline & Day1 & D2 & D3 & D4 & D5 & D6 & D7 & D8 & D9 & D10 & D11 & D12 & D13 & D14 \\
\hline Area5 & & & & & & & & & & & & 5 & 0 & 0 \\
\hline Area6 & & & & & 2 & 4 & 4 & 4 & 2 & 2 & 4 & 4 & 0 & 0 \\
\hline Area11 & & & & & & & & & & & 7 & 7 & 3 & 0 \\
\hline Area14 & & & & & & & & 6 & 5 & 6 & 1 & 6 & 0 & 0 \\
\hline
\end{tabular}

As seen in Table 4, Table 5, and Table 6, the hospitals start to reject patient type 1 on day 8 , and patient type 3 on day 13 (i.e. patients are admitted to the dummy hospital) due to resource shortages. The resource shortages which cause the rejections are determined and reported by the model, as shown in Table 7. It summarizes the healthcare system-wide resource cumulative shortages for all hospitals.

Table 7. Resource shortages

\begin{tabular}{|c|c|c|c|c|c|c|c|}
\hline Resource & Day8 & Day9 & Day10 & Day11 & Day12 & Day13 & Day14 \\
\hline Non-ICU Bed & -13 & -27 & -43 & -58 & -74 & -82 & -94 \\
\hline Ventilator & & & & & & -3 & -7 \\
\hline
\end{tabular}

Based on the resource shortages reported by the model, the decision maker can be alerted to request additional resources from an emergency agency. If additional resources become available, the model also helps to determine how to allocate the resources among hospitals in the healthcare network. Therefore, if the system obtains additional resources 
based on resource shortage prediction (i.e. 94 non-ICU beds, 7 ventilators), the model optimizes the allocation as shown in Table 8.

Table 8. Allocation of additional resources

\begin{tabular}{|c|c|c|c|c|c|c|c|}
\hline Allocation & $\begin{array}{c}\text { Hospital } \\
1\end{array}$ & $\begin{array}{c}\text { Hospital } \\
2\end{array}$ & $\begin{array}{c}\text { Hospital } \\
3\end{array}$ & $\begin{array}{c}\text { Hospital } \\
4\end{array}$ & $\begin{array}{c}\text { Hospital } \\
5\end{array}$ & $\begin{array}{c}\text { Hospital } \\
6\end{array}$ & $\begin{array}{c}\text { Hospital } \\
7\end{array}$ \\
\hline $\begin{array}{c}\text { Non-ICU } \\
\text { Bed }\end{array}$ & 66 & 0 & 14 & 0 & 0 & 0 & 14 \\
\hline Ventilator & 7 & 0 & 0 & 0 & 0 & 0 & 0 \\
\hline
\end{tabular}

With the allocation of additional resources among the healthcare facilities in Table 8, the patient allocation can be optimized as shown in Table 9, Table 10, and Table 11.

Table 9. Patient type 1 allocation summary with additional resource allocation

\begin{tabular}{|c|c|c|c|c|c|c|c|c|c|c|c|c|c|c|}
\hline & D1 & D2 & D3 & D4 & D5 & D6 & D7 & D8 & D9 & D10 & D11 & D12 & D13 & D14 \\
\hline A1 & 5 & 5 & 5 & 5 & 6 & 5 & 5 & 6 & 6 & 6 & 6 & 6 & 6 & 6 \\
\hline A2 & 2 & 2 & 2 & 2 & 2 & 2 & 2 & 2 & 2 & 6 & 6 & 6 & 6 & 6 \\
\hline A3 & 6 & 6 & 6 & 6 & 6 & 6 & 6 & 6 & 6 & 6 & 6 & 6 & 6 & 6 \\
\hline A4 & 4 & 4 & 4 & 4 & 4 & 4 & 4 & 4 & 4 & 4 & 4 & 4 & 4 & 4 \\
\hline A5 & 5 & 5 & 5 & 5 & 5 & 5 & 5 & 5 & 5 & 5,6 & 5 & 5 & 5,6 & 5 \\
\hline A6 & 1 & 1 & 1 & 1 & 1 & 1 & 1 & 1 & 1 & 1 & 1 & 1 & 1 & 1 \\
\hline A7 & 1 & 1 & 1 & 1 & 1 & 1 & 1 & 1 & 1 & 1 & 1 & 1 & 1 & 1 \\
\hline A8 & 2 & 2 & 2 & 2 & 2 & 2 & 2 & 2 & 2 & 2,6 & 2 & 2,6 & 2 & 2 \\
\hline A9 & 2 & 2 & 2 & 2 & 2 & 2 & 2 & 2 & 2 & 2 & 2 & 2 & 2 & 2 \\
\hline A10 & 1 & 1 & 1 & 1 & 1 & 1 & 1 & 1 & 1 & 2 & 2 & 1,2 & 2 & 2 \\
\hline A11 & 3 & 3 & 3 & 3 & 3 & 3 & 3 & 3 & 3 & 3 & 3 & 3 & 3 & 3 \\
\hline A12 & 7 & 7 & 7 & 7 & 7 & 7 & 7 & 7 & 7 & 7 & 7 & 7 & 7 & 7 \\
\hline A13 & 7 & 7 & 7 & 7 & 7 & 7 & 7 & 7 & 7 & 7 & 7 & 7 & 7 & 7 \\
\hline A14 & 1 & 1 & 1 & 1 & 1 & 1 & 1 & 1 & 1 & 1 & 1 & 1 & 1 & 1 \\
\hline A15 & 3 & 3 & 3 & 3 & 3 & 3 & 3 & 3 & 3 & 3,4 & 3,4 & 3 & 2,3 & 3 \\
\hline A16 & 3 & 3 & 3 & 3 & 4 & 3,4 & 3,4 & 3,4 & 3,4 & 4 & 4 & 4 & 4 & 4 \\
\hline
\end{tabular}

Table 10. Patient type 2 allocation summary with additional resource allocation

\begin{tabular}{|l|l|l|l|l|l|l|l|l|l|l|l|l|l|} 
Day1 & D2 & D3 & D4 & D5 & D6 & D7 & D8 & D9 & D10 & D11 & D12 & D13 & D14 \\
\hline
\end{tabular}




\begin{tabular}{|c|l|l|l|l|l|l|l|l|l|l|l|l|l|l|}
\hline Area5 & & & & & & & & & & & & 5 & 5 & 5 \\
\hline Area6 & & & & & 2 & 2 & 2 & 2 & 1 & 1 & 1 & 1 & 1 & 2 \\
\hline Area11 & & & & & & & & & & & 3 & 3 & 3 & 3 \\
\hline Area14 & & & & & & & & 1 & 1 & 1 & 1 & 1 & 1 & 1 \\
\hline
\end{tabular}

Table 11. Patient type 3 allocation summary with additional resource allocation

\begin{tabular}{|c|c|c|c|c|c|c|c|c|c|c|c|c|c|c|}
\hline & Day1 & D2 & D3 & D4 & D5 & D6 & D7 & D8 & D9 & D10 & D11 & D12 & D13 & D14 \\
\hline Area5 & & & & & & & & & & & & 5 & 6 & 5 \\
\hline Area6 & & & & & 4 & 4 & 4 & 6 & 2 & 6 & 2 & 2 & 4 & 1 \\
\hline Area11 & & & & & & & & & & & 7 & 7 & 3 & 4 \\
\hline Area14 & & & & & & & & 1 & 1 & 1 & 1 & 1 & 1 & 1 \\
\hline
\end{tabular}

As noted from the comparison between Table 4, Table 5, Table 6, Table 9, Table 10 and Table 11, the additional resource allocation can change patient allocation for the entire planning horizon. Since additional resources are allocated among hospitals to serve the patients, more patients are able to obtain improved service (i.e. admitted to a closer hospital). For example, without additional resources, as seen from Table 4, patients from area 6 are distributed among hospitals 1,2 and 6, and patients from area 7 are distributed among hospitals 5 and 6 and the dummy hospital (i.e. being rejected), while with additional resources, 66 non-ICU beds are assigned to hospital 1 as suggested in Table 8, and hospital 1 is the closest hospital for the patients from area 6 and area 7, as seen from Table 9, the patients from area 6 and area 7 are admitted to their closest hospital 1 with the additional resource allocation.

The numerical results presented above demonstrate how the model would help decision makers determine the patient allocation and potential resource shortages in the healthcare network for an estimated pandemic case in Metro-Louisville, KY with a most likely scenario of $35 \%$ gross attack rate and 6 weeks duration with $25 \%$ of the current total 
medical resource is dedicated to the pandemic patients. Additionally the model can be used to determine the allocation of additional resources to healthcare facilities. Since the gross attack rate, pandemic duration, and the proportion of the resources dedicated to pandemic patients are the three important assumptions which impact the solution, experimentation with different levels of these three factors is conducted. Table 12 shows the levels of each factor to be considered in the experiments.

Table 12. Different levels for each factor

\begin{tabular}{|c|c|c|c|}
\hline Factor & \multicolumn{3}{|c|}{ Levels } \\
\hline Gross Attack Rate & $15 \%$ & $25 \%$ & $35 \%$ \\
\hline Duration (in weeks) & 6 & 8 & 12 \\
\hline $\begin{array}{c}\text { Proportion of Hospital Resources } \\
\text { Dedicated to Pandemic Influenza Patients }\end{array}$ & $15 \%$ & $25 \%$ & $35 \%$ \\
\hline
\end{tabular}

The objective function of the model is to minimize the total travel distance between patients and hospitals. However, the objective value itself does not impart specific patient service and resource status information. Therefore the earliest resource shortage date is used to compare the experimental results. The result for each factor level combination is shown in Table 13.

Table 13. Resource shortage comparison

\begin{tabular}{|c|c|c|c|c|}
\hline $\begin{array}{c}\text { Gross } \\
\text { Attack } \\
\text { Rate }\end{array}$ & $\begin{array}{c}\text { Duration } \\
\text { (in weeks) }\end{array}$ & $\begin{array}{c}\text { Resource } \\
\text { Proportion }\end{array}$ & Resource Shortage & $\begin{array}{c}\text { Earliest Resource } \\
\text { Shortage Date }\end{array}$ \\
\hline $15 \%$ & 6 & $15 \%$ & Non-ICU Bed & Day 15 \\
\hline $15 \%$ & 6 & $25 \%$ & No Shortages & - \\
\hline $15 \%$ & 6 & $35 \%$ & No Shortages & - \\
\hline $15 \%$ & 8 & $15 \%$ & No-ICU Bed & Day 25 \\
\hline $15 \%$ & 8 & $25 \%$ & No Shortages & - \\
\hline $15 \%$ & 8 & $35 \%$ & No Shortages & - \\
\hline $15 \%$ & 12 & $15 \%$ & No Shortages & - \\
\hline $15 \%$ & 12 & $25 \%$ & No Shortages & \\
\hline
\end{tabular}




\begin{tabular}{|c|c|c|c|c|}
\hline $15 \%$ & 12 & $35 \%$ & No Shortages & - \\
\hline \multirow{2}{*}{$25 \%$} & \multirow{2}{*}{6} & \multirow{2}{*}{$15 \%$} & Non-ICU Bed & Day 5 \\
\hline & & & Ventilator & Day 20 \\
\hline $25 \%$ & 6 & $25 \%$ & Non-ICU Bed & Day 14 \\
\hline $25 \%$ & 6 & $35 \%$ & No Shortages & - \\
\hline $25 \%$ & 8 & $15 \%$ & Non-ICU Bed & Day 15 \\
\hline $25 \%$ & 8 & $25 \%$ & Non-ICU Bed & Day 25 \\
\hline $25 \%$ & 8 & $35 \%$ & No Shortages & - \\
\hline $25 \%$ & 12 & $15 \%$ & Non-ICU Bed & Day 30 \\
\hline $25 \%$ & 12 & $25 \%$ & No Shortages & - \\
\hline $25 \%$ & 12 & $35 \%$ & No Shortages & - \\
\hline \multirow{3}{*}{$35 \%$} & \multirow{3}{*}{6} & \multirow{3}{*}{$15 \%$} & Non-ICU Bed & Day 4 \\
\hline & & & Ventilator & Day 11 \\
\hline & & & ICU Bed & Day 21 \\
\hline \multirow{2}{*}{$35 \%$} & \multirow{2}{*}{6} & \multirow{2}{*}{$25 \%$} & Non-ICU Bed & Day 8 \\
\hline & & & Ventilator & Day 13 \\
\hline \multirow{2}{*}{$35 \%$} & \multirow{2}{*}{6} & \multirow{2}{*}{$35 \%$} & Ventilator & Day 14 \\
\hline & & & Non-ICU Bed & Day 15 \\
\hline \multirow{2}{*}{$35 \%$} & \multirow{2}{*}{8} & \multirow{2}{*}{$15 \%$} & Non-ICU Bed & Day 10 \\
\hline & & & Ventilator & Day 22 \\
\hline \multirow{2}{*}{$35 \%$} & \multirow{2}{*}{8} & \multirow{2}{*}{$25 \%$} & Non-ICU Bed & Day 17 \\
\hline & & & Ventilator & Day 23 \\
\hline \multirow{2}{*}{$35 \%$} & \multirow{2}{*}{8} & \multirow{2}{*}{$35 \%$} & Ventilator & Day 24 \\
\hline & & & Non-ICU Bed & Day 26 \\
\hline \multirow{2}{*}{$35 \%$} & \multirow{2}{*}{12} & \multirow{2}{*}{$15 \%$} & Non-ICU Bed & Day 23 \\
\hline & & & Ventilator & Day 40 \\
\hline $35 \%$ & 12 & $25 \%$ & Non-ICU Bed & Day 33 \\
\hline $35 \%$ & 12 & $35 \%$ & No Shortages & - \\
\hline
\end{tabular}

As expected, the gross attack rate, duration, and proportion of the resources dedicated to influenza patients all have an impact on resource shortage. As the default assumption in FluSurge, $85 \%$ of the patients require a non-ICU bed, therefore non-ICU beds become the critical resources in most of the scenarios in Table 13. Gross attack rate has a significant impact on the resource shortage, that is, the higher the gross attack rate, the more resource shortage. For example, as show in Table 13, 35\% gross attack rate causes a resource shortage for all the scenarios except one with 12 weeks duration and $35 \%$ of the 
resources dedicated to influenza patients; this scenario also causes a earlier resource shortage, such as a non-ICU bed shortage occurring on day 4 with $15 \%$ dedicated resources and 6 weeks duration. Duration has an impact as well. The longer the duration, the more that the patients are spread out during the planning horizon; the less likely a resource shortage is, the later the resource shortage occurs. The proportion of hospital resources dedicated to pandemic influenza patients is another important factor to impact the performance. Obviously, the higher the proportion dedicated to the pandemic patients, the larger the number of patients that are served. As shown in Table 13, when only $15 \%$ of hospital resources are dedicated to the patients, all the scenarios are under resource shortage except one that is the one with a $15 \%$ gross attack rate and 12 weeks duration.

As shown in Table 13, a resource shortage is highly likely during an influenza pandemic outbreak. In the event of a resource shortage, decision makers must determine the type and quantity of additional resources to be requested and the impact on patient service. Toward this end, a sensitivity analysis can be performed.

\subsection{Sensitivity Analysis}

In general, sensitivity analysis helps to determine how sensitive the optimal solution is to changes in data values, which includes analyzing changes in an objective function coefficient and a right hand side value of a constraint. For the patient allocation problem as well as the resource allocation problem, sensitivity analysis can determine how different quantities of additional resources would change the service to the patients. Therefore the impact of changes to the right hand side of constraints (25) and (26) in Model 5 in Chapter 3 will be studied. 
Using a sensitivity analysis, the following questions are expected to be answered:

1) Are there unused quantities of resources? If so, which resources and in what quantities?

2) How will additional quantities of each resource improve patient service (i.e. reduce total patient travel distance)? What is the range of allowable resource quantity increase (i.e. at what point does the increase of a resource not improve the patient service)?

3) Since some patients require more than one resource type, different types of resources are correlated. How will the simultaneous changes of several types of resources impact patient service?

4) Overall, what is the solution space in which every single resource can improve patient service (i.e. the increase of resource quantity can improve patient service)?

\subsubsection{Basic Sensitivity Analysis}

Usually optimization software, such as LINGO, gives a solution report including the sensitivity analysis. However these default solver software sensitivity analyses have the limitation of not providing answers to all the questions outlined above. The sensitivity report involves only 1 change at a time. Even though the $100 \%$ rule can be applied for multiple changes, this rule is limited by the fact that the simultaneous changes are constrained by the sum of the percentages of the changes divided by the corresponding maximum allowable change not exceeding 100\% (Ward \& Wendell, 1990). Even for the case of one change at a time, the sensitivity report only gives the allowable range for the right hand side value in which the shadow price does not change. However, the helpful 
information of the allowable range for a certain type resource is the range in which the service would be improved along the increase of the resource level, rather than constant shadow price. In other words, in the allowable range, the objective value improves as the resource level increases, no matter if the shadow price changes.

To demonstrate the limitation of basic sensitivity analysis, the case study in Section 4.1 was solved in LINGO and the sensitivity analysis was investigated. The resource shortage is shown in Table 7. The quantities of additional resources are the right hand side of constraints (25) and (26) in Model 5. The sensitivity analysis is expected to determine how different quantities of additional resources would change the service to the patients. From the LINGO report, the following information is obtained as in Table 14. The dual price in the report is the amount that the objective would improve as the right-hand side of the constraint is increased by one unit and is valid only over a range of values. Consider non-ICU beds as an example; 94 non-ICU beds are all allocated among the hospitals since the slack is 0 , and the dual price of 2.95 implies that the total patient travel distance would decrease 2.95 miles if the quantity of additional non-ICU beds is increased by one unit, and the dual price is only valid for one unit since the allowable increase is 1 . However, even if the quantity of additional non-ICU beds is increased more than one unit and the dual price is not valid, the objective function value would still be improved. It would be useful to determine the range of values for the right hand side to improve the objective function value rather than the range for valid dual price (i.e. the range of additional resources to improve the patient service). 
Table 14. Sensitivity analysis report from LINGO

\begin{tabular}{|c|c|c|c|c|c|}
\hline & $\begin{array}{c}\text { Right hand } \\
\text { side value }\end{array}$ & Slack/surplus & Dual price & $\begin{array}{c}\text { Allowable } \\
\text { increase }\end{array}$ & $\begin{array}{c}\text { Allowable } \\
\text { decrease }\end{array}$ \\
\hline Non-ICU & 94 & 0 & 2.95 & 1 & 0 \\
\hline ICU & 0 & 0 & 3.27 & 1 & 0 \\
\hline Ventilator & 7 & 0 & 9.60 & 0 & 0 \\
\hline
\end{tabular}

Therefore, several advanced sensitivity analysis methods are developed: two-point comparison, three-point comparison, and a combination of two-point and three-point comparisons as discussed below.

\subsubsection{Advanced Sensitivity Analysis}

As mentioned in Chapter 3.4, many healthcare resources may be depleted by the surge demand during a pandemic influenza outbreak and the models presented in this work can help predict these resource shortages. The resource shortages obtained from equations (23) and (24) in Chapter 3.4 are the minimum amount (i.e., lower bound) of additional resource quantities needed to be able to satisfy the patient demand. The maximum amount (i.e. upper bound) of additional resource quantities needed also can be calculated, which means the amount of each type of resource needed to best serve the patients, i.e. all the patients can be served in their closest hospital. Then all possible combinations of resource quantities between lower bound and upper bound of each type resource are under the consideration for sensitivity analysis.

Intuitively, the higher the resource quantities, the better the patients are served in terms of patients' travel distance. However, some resources are correlated with other resources (e.g. ICU bed and ventilator since some patients need both of them). The increase of some resources above certain amounts would not guarantee the improvement of the objective value (i.e. travel distance). In other words, once a resource quantity reaches a 
boundary, additional quantities of the resource will not improve the system performance while other resource quantities remain the same. Therefore, it would be helpful to determine the boundary to increase certain resource quantities to improve the system performance. Three methods are proposed in this chapter to investigate this boundary: two-point comparison, three-point comparison and combination of two-point and threepoint comparisons.

\subsubsection{1 two-point comparison}

The quantity increase of a certain resource will improve the system performance until the resource quantity reaches a level such that increasing the quantity above this level will not improve the performance since the quantity of other correlated resources will limit patient service. So as the quantity of a resource is increasing from its lower bound to its upper bound, while other resource quantities remain unchanged, the pattern of the system performance will improve to a certain point then plateau. This section presents a method to find the boundary by comparing a pair of two consecutive points of adjacent resource quantities. If the latter point (i.e. a point stands for a resource quantity) has a better performance than the former point, then form a new pair of two consecutive points, the first point is the latter point of the previous pair. Keep forming the pair of two consecutive points until the performance of the two points are equal or reach the upper bound (i.e. the maximum quantity needed to best serve patients), then the boundary is the first point of last pair, or the upper bound.

The two-point comparison method is very straightforward and easily implemented. However when the search range (between lower bound and upper bound) is relatively 
large and the boundary point (i.e. stopping point for the search) is near the upper bound, this method could be very time-consuming. Hence, another alternative method, the threepoint comparison, is considered as below.

\subsubsection{2 three-Point Comparison}

The three-point comparison method proposed in this section reduces the search range by half in each comparison iteration and determines the range the boundary point falls in.

In each comparison iteration, the search range is determined by two points (i.e. two levels of resource quantities); the start point and end point of the range. A third point is determined as the middle point of the range. (i.e. a point stands for a resource quantity) Then, the objective function value is calculated for the three points of the range: minimum point, middle point and maximum point. The three points divide the original search range into two half-size sub-ranges. The comparison among the three points determines which sub-range the boundary point falls in. If the end point has better performance than the middle point, then the boundary point falls in the latter sub-range, otherwise it falls in the former sub-range. Therefore this method reduces the size of the next search range in half. By continuing in this manner, the search size will be reduced small enough to obtain the boundary point.

If the initial search range (between lower bound and upper bound) is relatively large and the boundary point falls near the upper bound, the three-point comparison method will be more efficient than two-point comparison since it would require less iterations to reach the boundary point. 


\subsubsection{Combination of two-Points Comparison and three-Points Comparison}

For the case study introduced in section 4.1, ICU beds and ventilators are two correlated resources for patient types 2 and 3 . Between the lower and upper bounds of each resource type, all the possible combinations of ICU bed and ventilator quantities are considered in the sensitivity analysis. If the ICU bed level is kept constant, the two-point comparison or three-point comparison will calculate the boundary of the ventilator level beyond which more ventilators do not improve performance. In addition, as ICU bed level is increased, the search procedure for the boundary of ventilator is repeated for ICU bed quantities between the lower and upper bounds. As mentioned above, if the initial search range (between lower bound and upper bound) is relatively large and the boundary point falls in near end of the search range, the three-point comparison method would be more efficient than two-point comparison since it would require less iterations to reach the boundary point. However, it also might be time-consuming to repeat the three-point comparison search procedure for each ICU bed quantity. Intuitively, as the ICU bed quantity is increased, the boundary of ventilators remains the same or increases since more ICU beds can support the increase usage of ventilators. Therefore the combination of two-point and three-point comparisons can be used. For example, the ICU bed quantity starts at its lower bound and three-point method is used to find the boundary point for ventilators. As ICU bed quantity is increased by one until the upper bound is reached, the search procedure is applied using two-point comparison and the starting point of the first pair comparison is the boundary point from the previous ICU quantity search procedure. Since the increase of the boundary for ventilators at each step as the ICU beds are 
increased by one is very small ( 0 or 1 ), the two-point comparison requires fewer iterations than the three-point comparison to find the boundary.

The quantity of ICU beds is kept constant and the search procedure is used to find the boundary point for ventilators as explained above. Similarly, the quantity of ventilators is kept constant to find the boundary for ICU beds.

\subsubsection{Numerical Results of Sensitivity Analysis}

The sensitivity analysis of the case study introduced in section 4.2 is presented here. This sensitivity analysis will help determine the impact of different quantities of additional resources on the system patient service in terms of travel distance.

As discussed in section 4.2.2, all the possible combinations of different quantities of each type resource between the lower and upper bounds are under consideration in the sensitivity analysis. As the quantity of one resource type increases from its lower bound to its upper bound, the two-point and three-point methods can be used to find the boundary point beyond which the system performance will not be improved. Since all the possible combinations of different quantities of each resource type need to be examined, the simultaneous changes of different types of resources need to be investigated as well.

In this case study, as seen from Table 7, the system would incur a resource shortage of 94 non-ICU bed and 7 ventilators. Then it is assumed that additional resources become available and Model 5 from Chapter 3 can help to determine the allocation of additional resources among health care facilities. The quantities of additional resources are the right hand side of constraints (25) and (26) in Model 5 in Chapter 3. The sensitivity analysis is expected to determine how different quantities of additional resources would change the 
service to the patients. The values of resource shortage represent the lower bound for each resource type, i.e. the minimum additional resource quantities needed to satisfy the patient demand. In addition, the upper bound is obtained as the maximum additional resource quantities needed to best serve the patients (i.e. each patient is assigned to the closest hospital). Upper bounds for this case study are 157 non-ICU beds, 14 ICU beds, and 20 ventilators. The upper bounds represent the maximum additional quantities of each resource type needed to best serve patients (i.e. assign all patients to their closest hospital). The lower and upper bounds for each resource type are summarized in Table 15.

Table 15. Lower bound and upper bound of each type resource level

\begin{tabular}{|c|c|c|c|}
\hline & Non-ICU bed & ICU bed & Ventilator \\
\hline Lower bound & 94 & 0 & 7 \\
\hline Upper bound & 157 & 14 & 20 \\
\hline
\end{tabular}

Since non-ICU beds are required by patient type 1 only, there is no correlation with the other resource types. Therefore, the increase of non-ICU bed quantities to the upper bound will always improve the performance. Since ICU beds and ventilators are correlated, the boundaries of both resource types can be found using sensitivity analysis. All the possible combinations of resource quantities between lower and upper bounds for both resource types are under investigation, therefore there are $(14-0+1) *(20-7+1)=210$ possible points.

Starting with the point ( 0 ICU beds, 7 ventilators), if additional ICU bed quantity remains at 0 , the boundary for increasing ventilators is found to be 17 , meaning increasing the quantity of ventilators above 17 does not improve system performance while the quantity 
of ICU beds remains at 0 . Similarly, if the ventilator quantity remains at 7 , the boundary for increasing ICU beds is found to be 5 . The next step in the sensitivity analysis search increases the number of ICU beds and ventilators by 1 . This search method continues until the ventilator quantity reaches its upper bound of 20 . The search range for ventilators $(20-7+1)=14$ is smaller than the search range for ICU beds $(14-0+1)=15$, therefore ventilators reach their upper bound faster than ICU beds. Then the search process stops since it considers all the possible combinations.

Table 16 shows the results for the sensitivity analysis for the case study which has the search range of ICU bed $[0,14]$, ventilator $[7,20]$. It includes the starting point for each search iteration, objective function value of the starting point, boundary level and corresponding objective function value. The objective function value is the total patient travel distance (miles). Since non-ICU bed is not related to ICU bed and ventilator in terms of patient types 2 and 3 , it is kept constant at 97.

Table 16. Boundary summary

\begin{tabular}{|c|c|c|c|c|c|c|}
\hline \multirow[b]{2}{*}{$\begin{array}{l}\text { Starting } \\
\text { ICU bed } \\
\text { level }\end{array}$} & \multirow[b]{2}{*}{$\begin{array}{c}\text { Starting } \\
\text { ventilator } \\
\text { level }\end{array}$} & \multirow{2}{*}{$\begin{array}{l}\text { Obj fn. } \\
\text { value } \\
\text { for } \\
\text { staring } \\
\text { point }\end{array}$} & \multicolumn{2}{|c|}{ ICU bed level kept constant } & \multicolumn{2}{|c|}{ Ventilator level kept constant } \\
\hline & & & $\begin{array}{c}\text { Boundary } \\
\text { level } \\
\text { for ventilator }\end{array}$ & $\begin{array}{l}\text { Obj. fn. } \\
\text { value for } \\
\text { boundary } \\
\text { point }\end{array}$ & $\begin{array}{c}\text { Boundary } \\
\text { level for ICU } \\
\text { bed }\end{array}$ & $\begin{array}{c}\text { Obj. fn. value } \\
\text { for boundary } \\
\text { point }\end{array}$ \\
\hline 0 & 7 & 2993.63 & 17 & 2960.75 & 5 & 2977.29 \\
\hline 1 & 8 & 2980.76 & 17 & 2957.48 & 6 & 2964.42 \\
\hline 2 & 9 & 2967.89 & 17 & 2954.22 & 7 & 2951.55 \\
\hline 3 & 10 & 2960.21 & 17 & 2950.95 & 8 & 2943.87 \\
\hline 4 & 11 & 2952.54 & 17 & 2947.68 & 9 & 2936.20 \\
\hline 5 & 12 & 2947.70 & 17 & 2944.41 & 10 & 2931.36 \\
\hline 6 & 13 & 2942.87 & 17 & 2941.15 & 11 & 2926.53 \\
\hline 7 & 14 & 2939.16 & 17 & 2937.88 & 12 & 2923.26 \\
\hline 8 & 15 & 2935.46 & 17 & 2934.61 & 13 & 2920.00 \\
\hline 9 & 16 & 2931.75 & 17 & 2931.34 & 14 & 2916.73 \\
\hline
\end{tabular}




\begin{tabular}{|l|l|l|l|l|l|l|}
\hline 10 & 17 & 2928.07 & 17 & 2928.07 & 14 & 2916.29 \\
\hline 11 & 18 & 2924.81 & 18 & 2924.81 & 14 & 2915.85 \\
\hline 12 & 19 & 2921.54 & 19 & 2921.54 & 14 & 2915.41 \\
\hline 13 & 20 & 2918.27 & 20 & 2918.27 & 14 & 2915.00 \\
\hline
\end{tabular}

Based on the boundary results shown in Table 16, the solution space can be generated in which resource quantities improve the patient service. In other words, every solution in the space is not dominated by others. The solution space for additional ICU beds and ventilators quantities is shown in Figure 2. For example, 2 points of (11 ICU beds and 14 ventilators) and (12 ICU beds and 14 ventilators) are included in the solution space, which means the point of 12 ICU beds gives a performance improvement compared to 11 ICU beds, while the point of (13 ICU beds and 14 ventilators) is not included in the solution space, which means that 13 ICU beds will not obtain performance improvement compared to 12 ICU beds due to the amount of ventilators. 


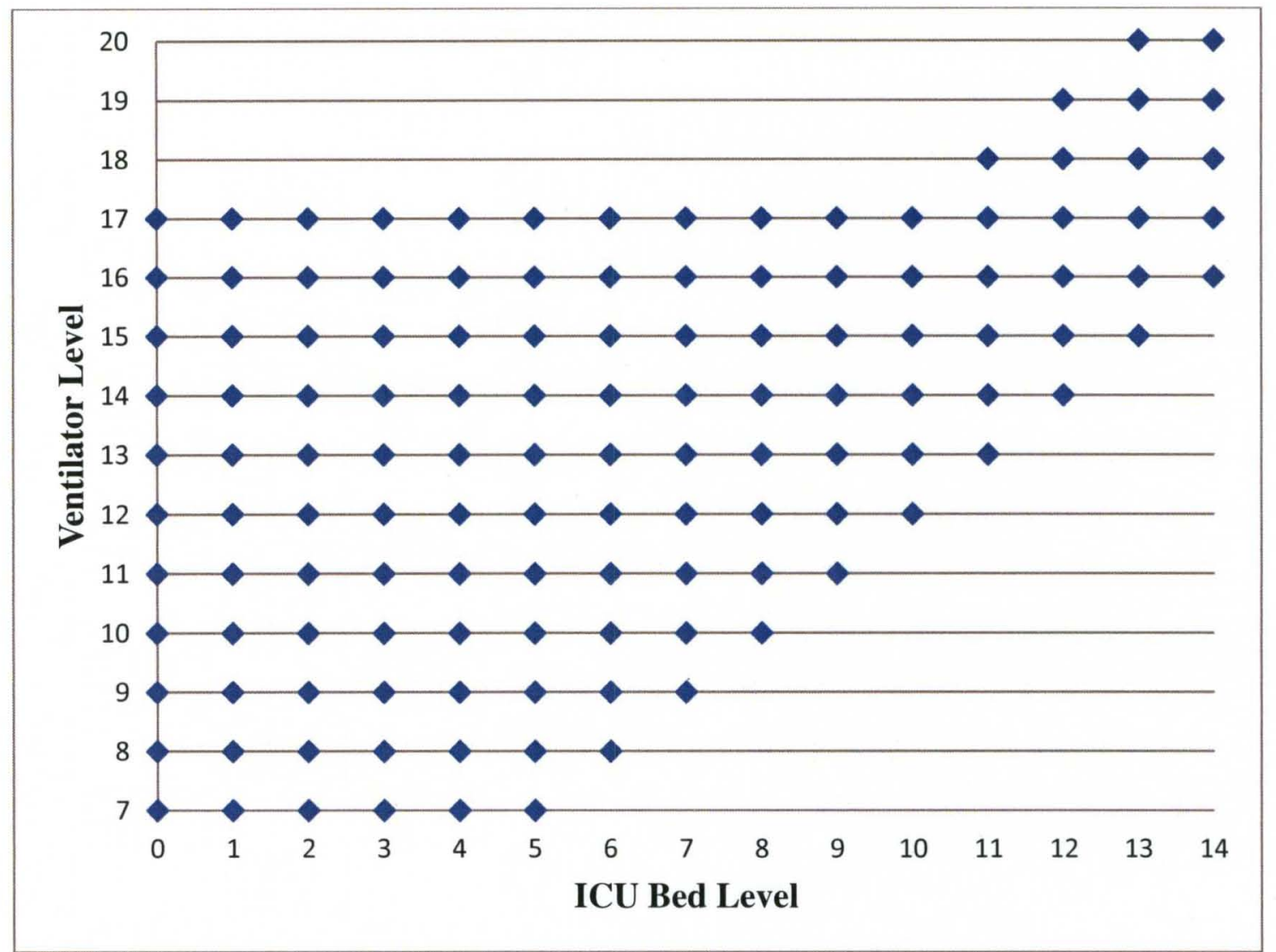

Figure 2. Sensitivity analysis solution space

The computation time to complete the sensitivity analysis and generate the Figure 2 solution space is compared among different methods using Matlab R2010a on a Pentium 4 PC with 1 GB memory and shown in Table 17.

Table 17. Computer run times for sensitivity analysis methods

\begin{tabular}{|c|c|}
\hline Sensitivity Analysis Method & Run time (minutes) \\
\hline two-point comparison & 10.07 \\
\hline three-point comparison & 6.66 \\
\hline Combination of two-point \& three-point comparison & 3.07 \\
\hline
\end{tabular}

As mentioned above, the sensitivity analysis search range is from the lower to upper bound of each resource type, which could be very large. In reality, obtaining a large 
additional quantity of resources may not be possible. Therefore, the search range can be modified by the users to consider a more reasonable and smaller range of possible additional resource quantities. In this way, the search time will be reduced. The decision makers can use sensitivity analysis to determine how different quantities of additional resources would change the service to the patients.

\subsection{Interface of Model Implementation}

A web-based application was developed by the members of a research team to include the models presented in the dissertation (Kelley et al., 2011). The users have the capability to change the input assumptions, such as the pandemic scenario, dedicated medical resources levels, etc. Additionally, the web-based tool can be used to perform what-if analysis. The screen shots (Figures 3 through 10) are attached below to demonstrate how the web-based tool can assist decision makers.

Figure 3 shows the planning input. Users can choose the planning horizon, planning regions, and planning hospitals. Figure 4 shows the map of the planning regions and hospitals. Figure 5 shows patient categories and resources required by each patient category. Figure 6 shows the demand assumptions. Users can choose the pandemic duration, gross attack rate, scenario (i.e. most likely, minimum, maximum), and proportion of each type patient, then demand is updated based on the input. Figure 7 shows the resource capacity, including both equipment and personnel resources. Users can update the resource capacity. Figure 8 shows the result for the patient allocation, i.e. how many patients from an area is assigned to a specific hospital. Figure 9 shows the resource shortage if there is any, i.e. how many additional resources are required at a 
certain day to meet the demand. Figure 10 shows the additional resources allocation. Based on the resource shortage predication from Figure 9, users are alerted to require more resources from other emergency agencies. Users can input the amount of additional resources, then the model gives the results for how to allocation the resources among hospitals.

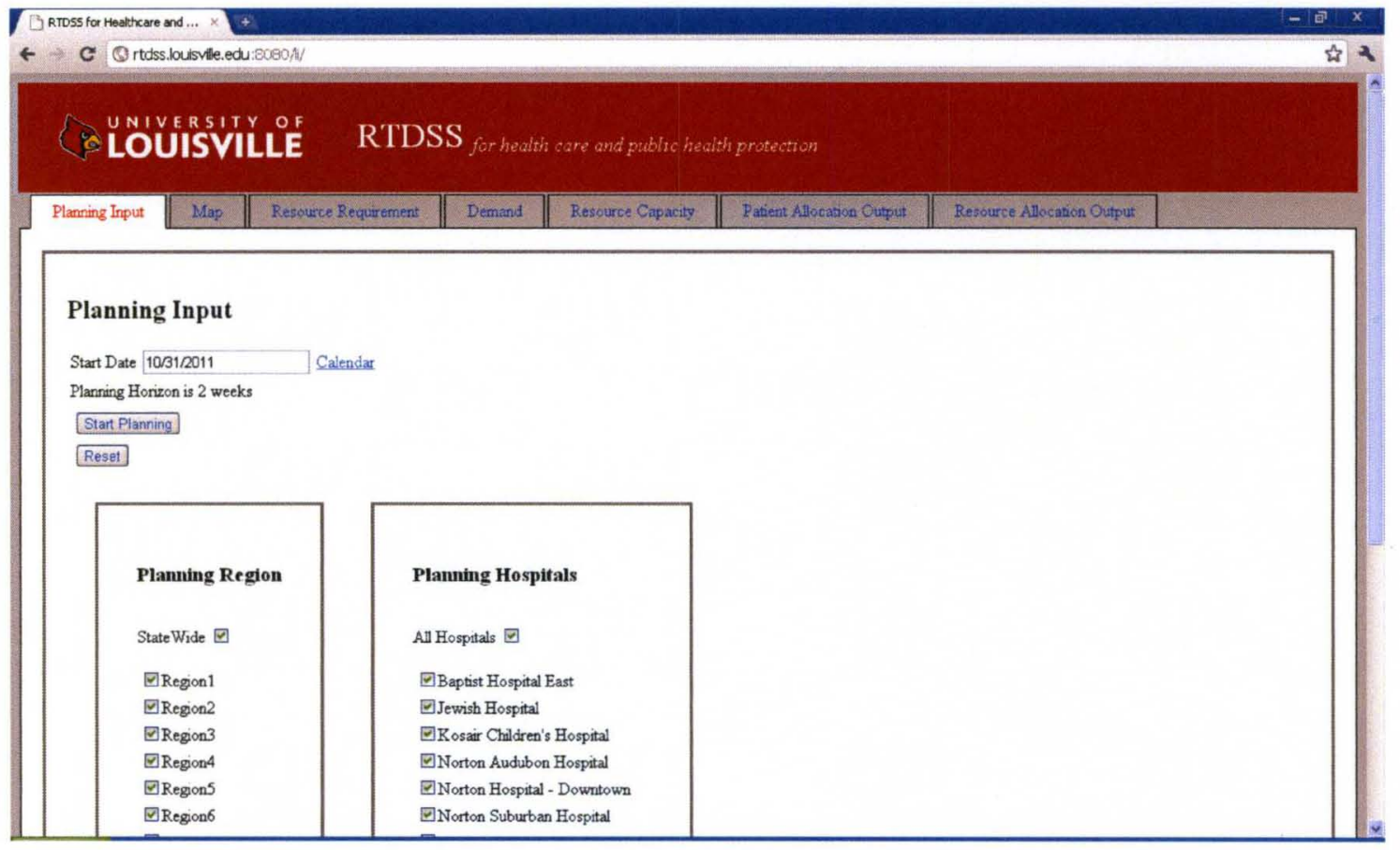

Figure 3: Planning input 


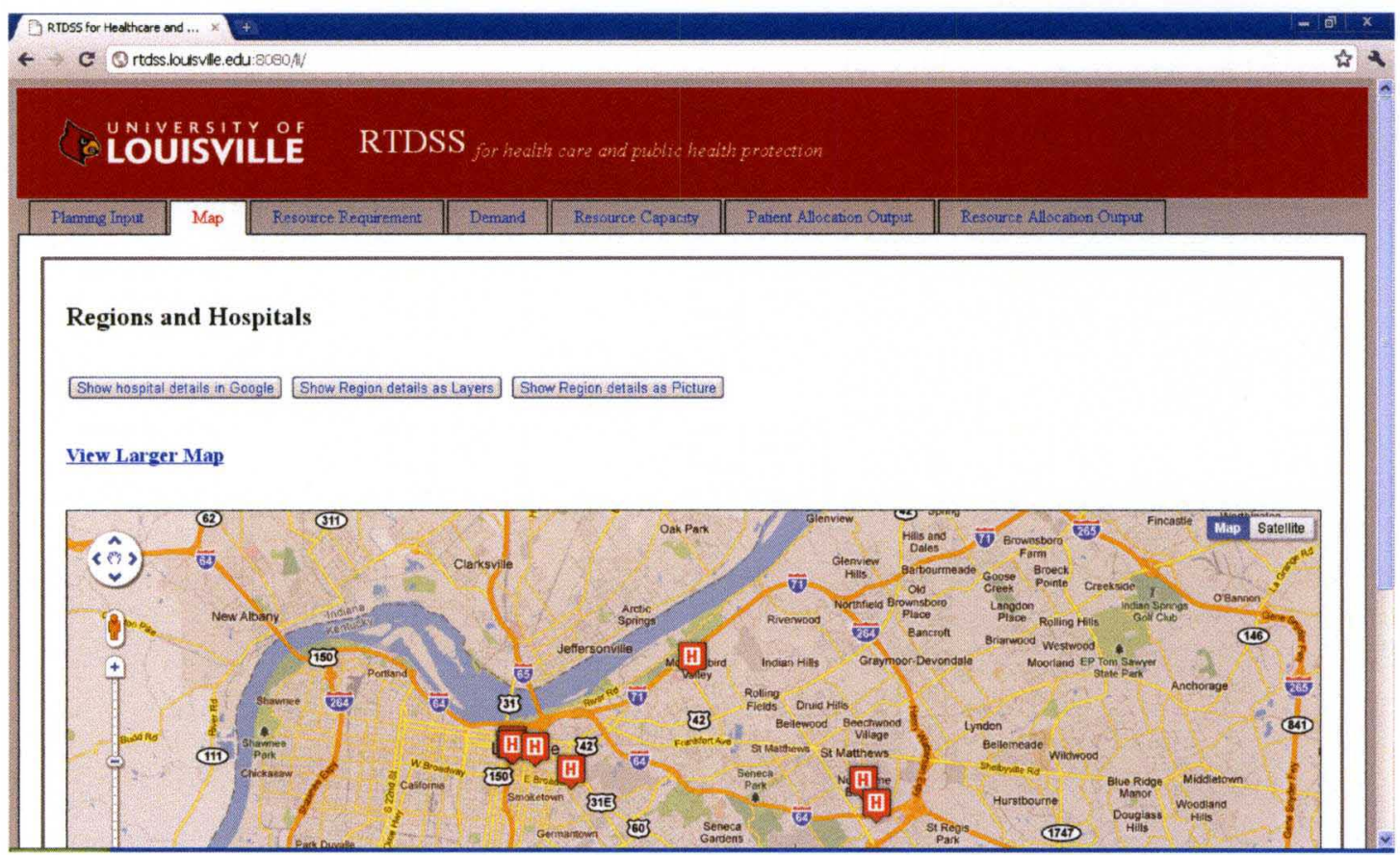

Figure 4. Map of regions and hospitals

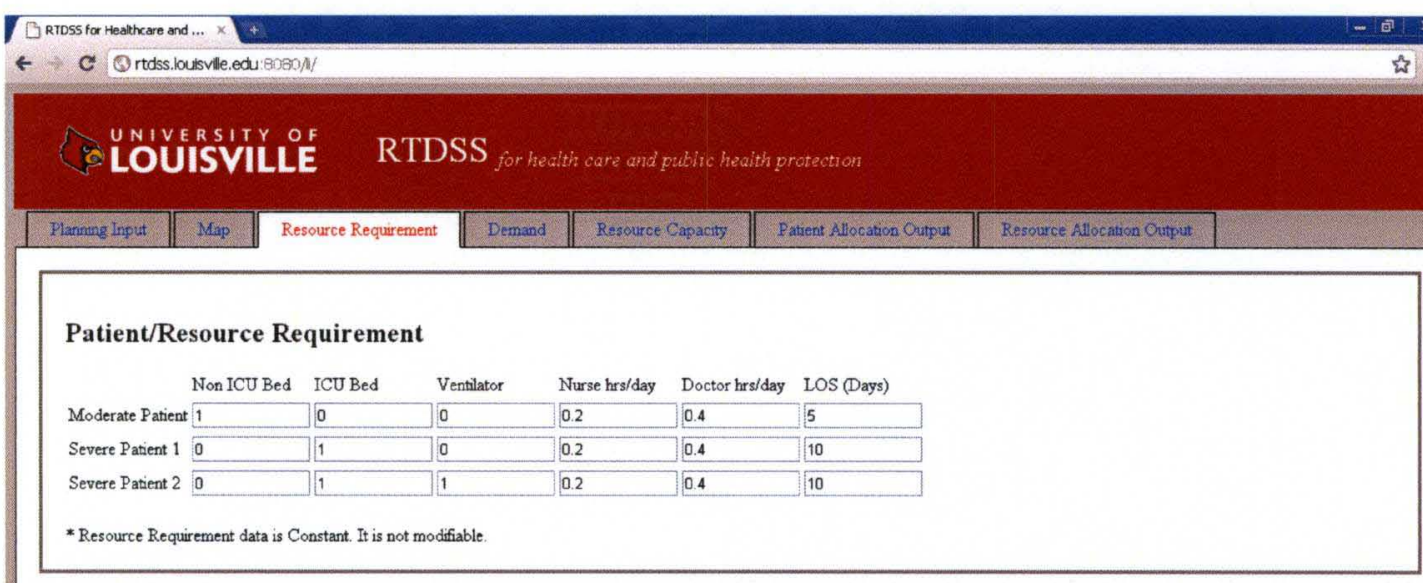

Figure 5. Patient/resource requirements 


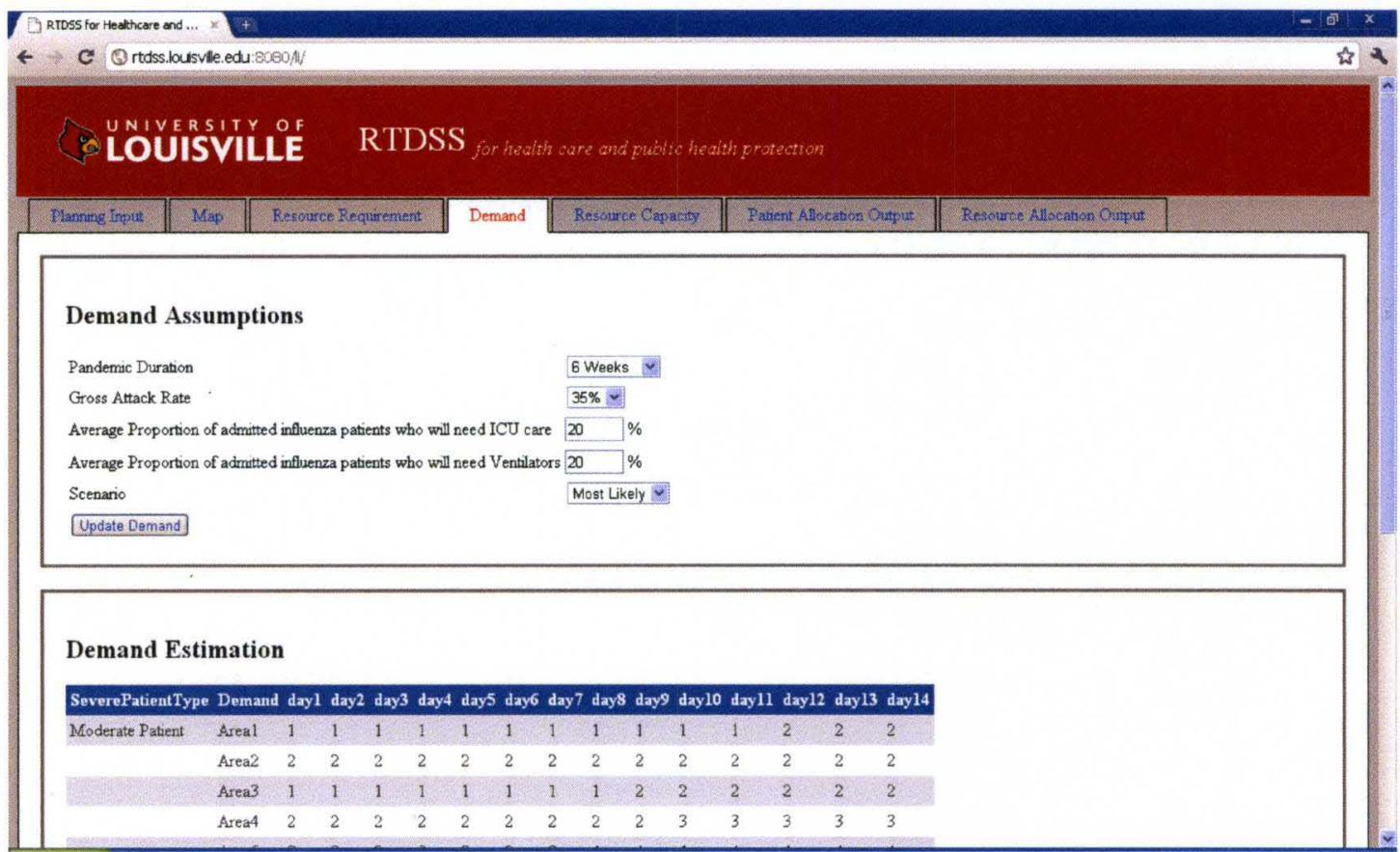

Figure 6. Demand assumptions

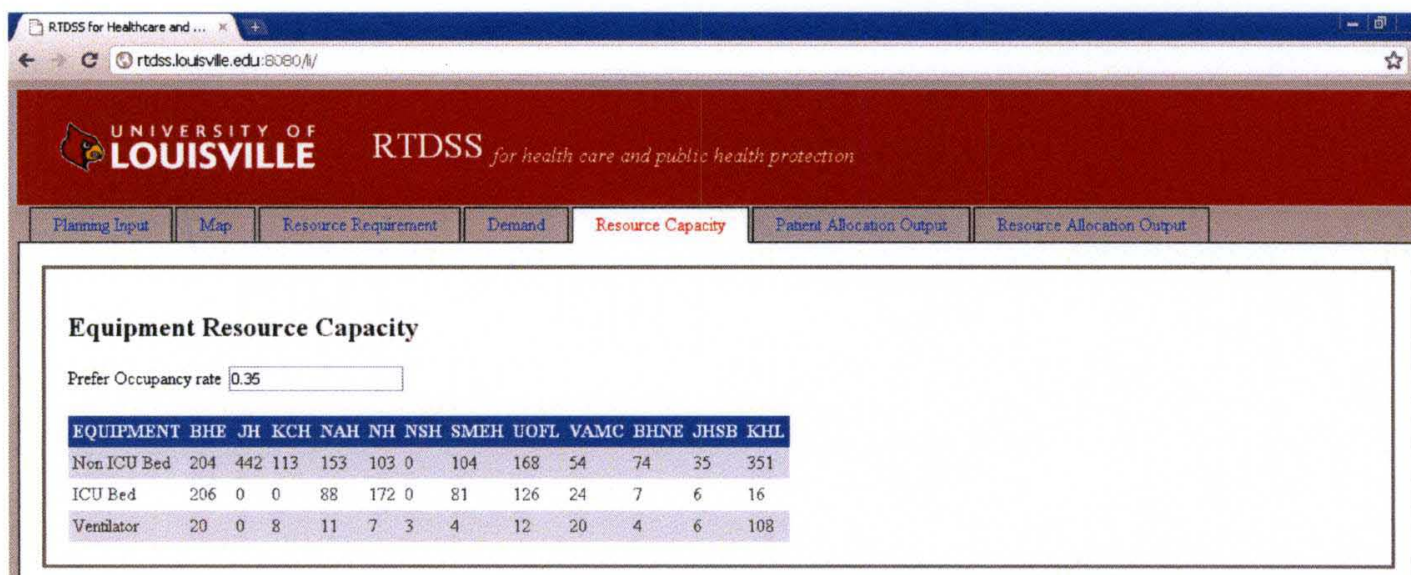

Personnel Resource Capacity

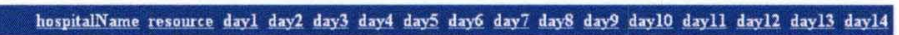

\begin{tabular}{llllllllllllllll}
\hline Edit BHE & Doctor & 15 & 15 & 15 & 15 & 15 & 15 & 15 & 15 & 15 & 15 & 15 & 15 & 15 & 15
\end{tabular}

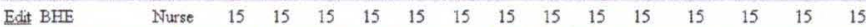

\begin{tabular}{llllllllllllllll}
\hline Edit JH & Doctor & 15 & 15 & 15 & 15 & 15 & 15 & 15 & 15 & 15 & 15 & 15 & 15 & 15 & 15
\end{tabular}

$\begin{array}{llllllllllllllll}\text { Edit IH } & \text { Nurse } & 15 & 15 & 15 & 15 & 15 & 15 & 15 & 15 & 15 & 15 & 15 & 15 & 15 & 15\end{array}$

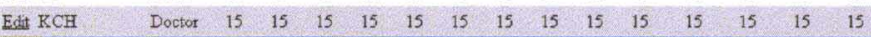

Figure 7. Resource capacity input 


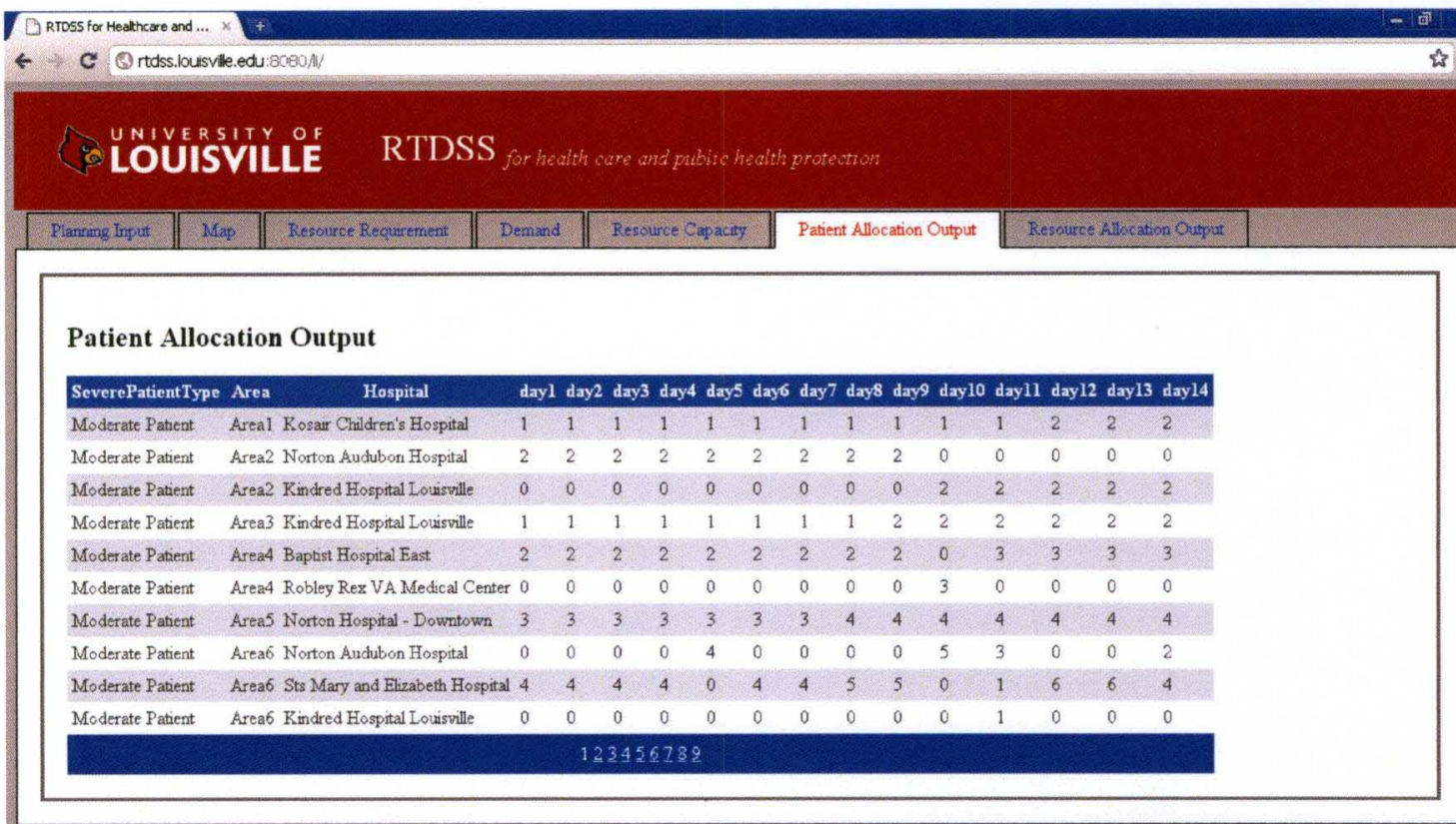

\section{Copyright (9) University of Louisville. All rights reserved}

Figure 8. Patient allocation output

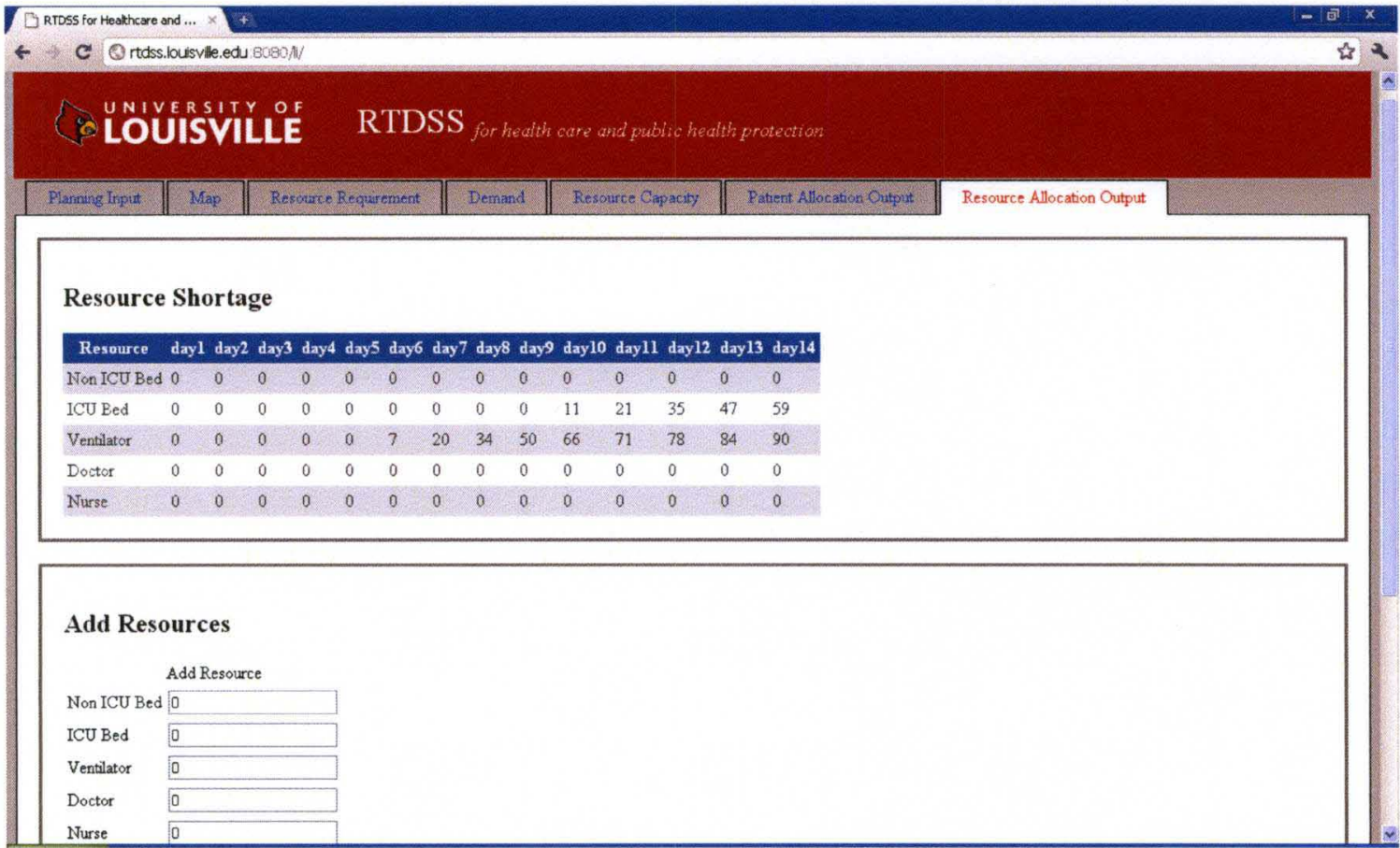

Figure 9. Resource shortage 


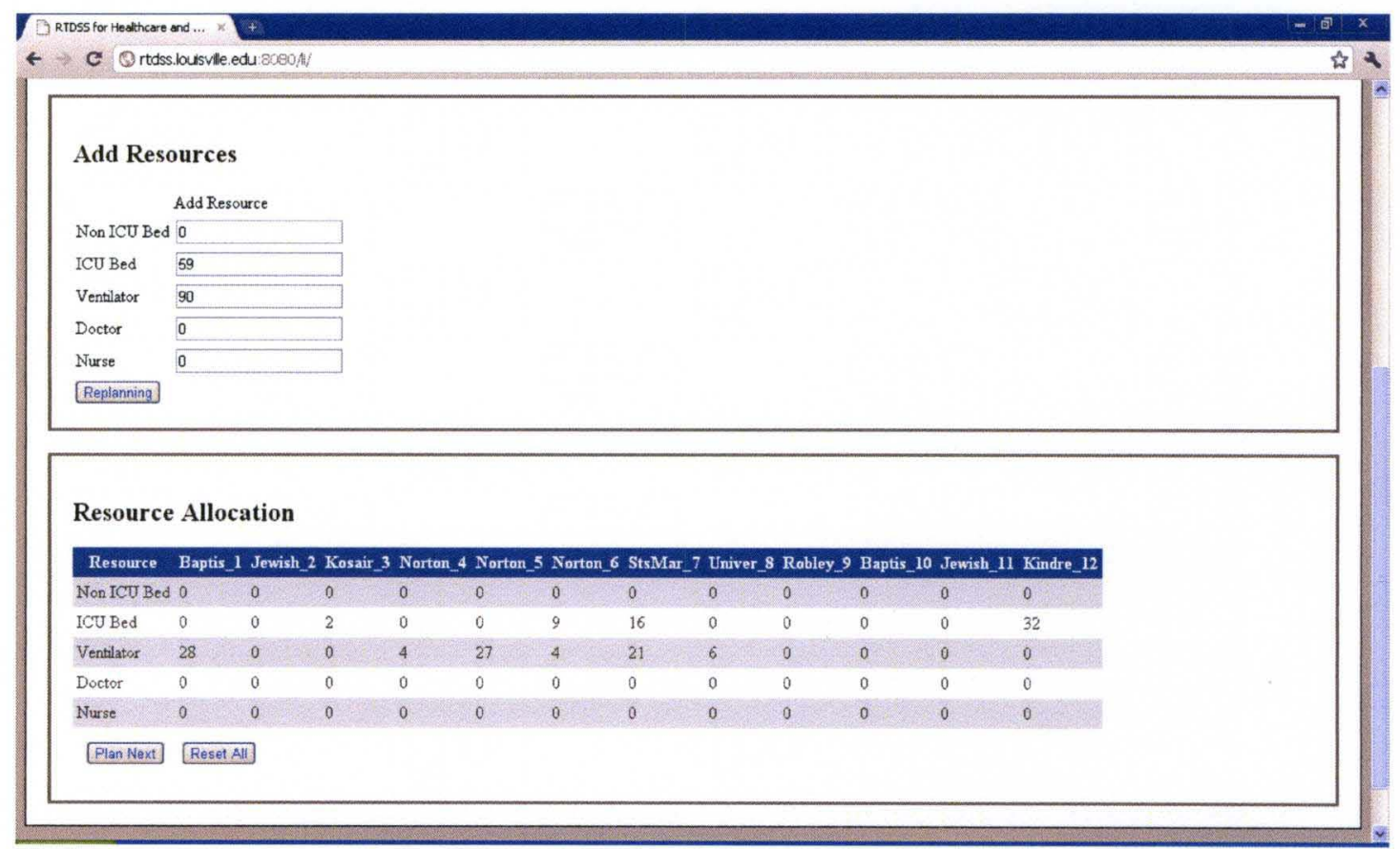

Figure 10. Additional resource allocation 


\section{CHAPTER 5 MULTI-OBJECTIVE OPTIMIZATION MODELS}

The models present in Chapter 3 are single-objective optimization models. The basic assumption is that the service quality of each healthcare facility is equitable and therefore the main concern of the patients is minimizing their travel distance. The single-objective is to minimize the total travel distance of all patients. In this chapter, multi-objective models are presented. The multi-objective nature of this problem relates to both the equity and efficiency of patient allocation among healthcare facilities. The two objectives conflict to some extent. An efficient system can be measured by the total travel distance between patients and healthcare facilities where the minimization of this total distance is

the goal. However, a strictly efficient system may result in some patients incurring a relatively high cost or travel distance. Patients might incur a huge difference of service access cost based on where they reside. The equity objective is to obtain a more equitable distribution of "cost", i.e. travel distance among patients, which is expressed as the goal of minimizing the maximum distance that patients travel to access the healthcare facilities.

The mathematical model presented in this chapter is developed to optimize the patient allocation among healthcare facilities considering two objectives related to patients' cost of access to healthcare services: 1) minimization of the total travel distance by patients to 
hospitals; and 2) minimization of the maximum travel distance between patients and hospitals. As with the previous single-objective optimization models, the multi-objective model presented in this chapter predicts resource shortages during the planning horizon. In addition, the multi-objective model aids in the determination of the optimal allocation of the additional resources, when available, among hospitals by considering both objectives related to patients' cost of access to services. A case study from Metro Louisville, Kentucky is presented to demonstrate how the multi-objective model would aid in patient allocation during a pandemic influenza outbreak.

\subsection{Multi-Objective Patient Allocation Model}

As mentioned in Chapter 3, the models developed in this research assume that the patient demand and healthcare sites resource information can be obtained. Based on the demand and capacity information, the multi-objective model optimizes the patient allocation by considering two objectives: 1) minimization of the total patient travel distance between population areas and healthcare facilities 2) minimization of the maximum patient travel distance to allow a more equitable distribution of "cost," i.e. travel distance among patients. Multi-objective optimization model is build based on Models 4 in Chapter 3, to optimize the patient allocation with the consideration of possible resource shortage. Compared to the single-objective optimization model, additional variables are needed to conduct the second objective to minimize the maximum patient travel distance, as shown below.

Decision variables:

$Y_{a h}=1$ if patients from area $a$ are assigned to hospital $h$, otherwise 0 
D maximum distance from patients to an assigned hospital

Second objective function:

$\operatorname{Min} D$

Constraints related to second objective

Distance $_{a, h} Y_{a, h} \leq D$

$\forall a, h(30)$

$\sum_{p} \sum_{t} X_{p, a, h, t} \leq M^{*} Y_{a, h}$

$\forall a, h(31)$

$X_{p, a, h, t} \geq 0$

$\forall p, a, h, t(32)$

$Y_{a, h}=0$ or 1

$\forall a, h(33)$

Second objective is defined in equation (29). Constraints (30) through (33) are the constraints to include the second objective to minimize the maximum travel distance of a patient.

If the healthcare facilities have resource shortages during the outbreak and additional resources become available, the feature to optimize the allocation of additional new resources can be added in the model as well, considering the same two objectives related to patients' cost of access to services. Additional parameters and decision variables, compared to above model, are added, which are the same as in Model 5 in Chapter 3.

There are two general approaches for solving the multi-objectvie optimization problem. One solution technique is to combine the multiple objectives to a single composite function or move all but one objective to the constraint set. The other approach is to 
generate a set of efficient solutions and allow the decision maker to evaluate the solutions and choose a desirable course of action. In this study, the first approach is used to solve the problem. There are many ways to transform a multi-objective problem to a singleobjective problem, including weighted-sum approach, goal programming, and constraint method. In this study the constraint method, which is the most commonly-used classical method of moving all but one objective to the constraint set, is employed to reformulate the multi-objective optimization problem to a single-objective function by keeping just one of the objectives and restricting the rest of the objectives within user-specified values (Deb, 2001). Using this method, the first objective is to be optimized while objective 2 is constrained to values that vary through a range of feasible values.

The constraint method requires an upper limit with respect to patient travel distance to a hospital. Additional parameters are used below to conduct the constraint method.

$$
\begin{aligned}
& \text { Distance }_{\max } \\
& \text { the pre-specified threshold value for the maximum allowable travel } \\
& \text { distance between patients and healthcare facilities }^{\text {Distance }_{\text {ah }}} \\
& \begin{array}{l}
\text { Distance }_{a h}=\text { Distance }_{a h} \text { if } \text { Distance }_{a h} \leq \text { Distance }_{\max } \text {, otherwise } \\
\text { Distance }_{a h}{ }_{a h}=\infty
\end{array}
\end{aligned}
$$

Then the multi-objective model is converted into a single objective problem by: 1) replacing Distance ${ }_{a h}$ with Distance $_{a h}$ in the first objective; 2) removing the second objective and the constraints which are associated with second objective (i.e. constraints (30), (31) and (33)).

\subsection{Case Study}


A same case study as in section 4.1 is conducted for the multi-objective model. A pandemic with $35 \%$ gross clinical attack rate and 6-week duration is considered in Louisville-Jefferson, $\mathrm{KY}$, and $25 \%$ of the hospital resources are dedicated to pandemic patients. The results in section 4.1 show that the system would occur non-ICU bed shortage from day 8 and ventilator shortage from day 13 from single-objective function, as seen from Table 7. To demonstrate how the multi-objective problem changes the results from single-objective problem. Both single-objective and multi-objective models are solved for the first week before the shortage occurs to see how the patient allocation results are different. Then both models are solved for the second week to see how the models obtain different results for resource shortage and additional resource allocation.

Initially the model is used to generate a solution for the first week of the 6-week pandemic outbreak with the consideration of only the first objective to minimize the total travel distance. The model was solved in seconds using LINGO 11.0 on a Pentium 4 PC with 1 GB memory. To explain how the model can help decide the patient allocation, consider patient type 1 as an example to demonstrate the results. The allocation results are summarized in Table 18 which shows to which hospitals Type 1 patients from each area should be allocated. For example, new hospital admits of influenza Type 1 patients on day 5 from area 14 should be assigned to hospitals 1 and 6. As stated earlier, it is assumed these Type 1 patients will occupy a non-ICU bed for 5 days.

Table 18. Patient type 1 allocation summary for single objective

\begin{tabular}{|c|c|c|c|c|c|c|c|}
\hline ToHospitalSummary & Day 1 & Day 2 & Day 3 & Day 4 & Day 5 & Day 6 & Day 7 \\
\hline Area 1 & 5 & 5 & 6 & 6 & 6 & 6 & 6 \\
\hline Area 2 & 2,4 & 4 & 4 & 4 & 4 & 4 & 4 \\
\hline Area 3 & 6 & 6 & 6 & 6 & 6 & 6 & 6 \\
\hline
\end{tabular}




\begin{tabular}{|c|c|c|c|c|c|c|c|}
\hline Area 4 & 4 & 4 & 4 & 4 & 4 & 4 & 4 \\
\hline Area 5 & 5 & 5 & 5 & 5 & 5 & 5 & 5 \\
\hline Area 6 & 1 & 1 & 6 & 2 & 2 & 1 & 1 \\
\hline Area 7 & 1 & 1 & 6 & 6 & 6 & 5 & 5 \\
\hline Area 8 & 2 & 2 & 2 & 2 & 2 & 2 & 2 \\
\hline Area 9 & 2 & 2 & 4 & 4 & 2 & 2 & 2 \\
\hline Area 10 & 2 & 2 & 2 & 2 & 2 & 2 & 2 \\
\hline Area 11 & 3 & 3 & 3 & 3 & 3 & 3 & 3 \\
\hline Area 12 & 7 & 7 & 7 & 7 & 7 & 7 & 7 \\
\hline Area 13 & 7 & 7 & 7 & 7 & 4,7 & 7 & 7 \\
\hline Area 14 & 1 & 1 & 6 & 6 & 1,6 & 1 & 1 \\
\hline Area 15 & 3 & 3 & 4 & 4 & 3 & 3 & 3 \\
\hline Area 16 & 3 & 3 & 4 & 4 & 4 & 3,4 & 3,4 \\
\hline
\end{tabular}

Without considering the second objective to minimize the maximum patient travel distance, the total travel distance (objective 1) is 1325.03 miles which means that the average patient travel distance is 3.98 miles/patient $(1325.03$ miles $/ 333$ patients $=3.98$ miles/patient), while the maximum distance is 11.37 miles for the patients who are from area 14 assigned to hospital 6. This maximum distance of 11.37 miles is almost three times the average travel distance. Therefore the model with the consideration of only the objective to minimize the total travel distance gives a solution with a wide variance of individual patient costs. Hence, the second objective is added to the model to minimize the maximum patient travel distance which helps to make the costs more equitable between patients.

Table 19. Distance (miles) for the single objective of minimization of total travel distance

\begin{tabular}{|c|c|c|}
\hline Total Distance & Average Distance & Maximum Distance \\
\hline 1325.03 & 3.98 & 11.37 \\
\hline
\end{tabular}


As mentioned, the constraint method is used to solve the multi-objective patient allocation model. This method requires an upper limit, which is pre-defined by users, for the patient travel distance to a hospital. In the model the user defined maximum distance Distance $_{\max }$ and the definitions of Distance' ${ }_{a h}$ illustrate the upper limit since Distance' ${ }_{a h}$ $=$ Distance $_{a h}$ if Distance $_{a h} \leq$ Distance $_{\max }$, otherwise Distance $_{a h}=\infty$. The upper limit is provided by the decision makers to perform a what-if analysis by changing the upper limit within a certain range. For demonstration purpose, an upper limit of 9 miles (i.e. Distance $_{\max }=9$ miles) was chosen to show the results and compare them to the singleobjective model. The patient allocation and travel distance summary of Type 1 patients for this multi-objective model are shown in Table 20. Again, the mathematical model was solved in seconds using LINGO 11.0 on a Pentium 4 PC with 1 GB memory.

Table 20. Patient type 1 allocation summary for multi-objective patient allocation model

\begin{tabular}{|c|c|c|c|c|c|c|c|}
\hline ToHospitalSummary & Day 1 & Day 2 & Day 3 & Day 4 & Day 5 & Day 6 & Day 7 \\
\hline Area 1 & 5 & 5 & 6 & 6 & 6 & 6 & 6 \\
\hline Area 2 & 2 & 2 & 4 & 4 & 4 & 4 & 4 \\
\hline Area 3 & 6 & 6 & 6 & 6 & 6 & 6 & 6 \\
\hline Area 4 & 4 & 4 & 4 & 4 & 4 & 4 & 4 \\
\hline Area 5 & 5 & 5 & 5 & 5 & 5 & 5 & 5 \\
\hline Area 6 & 1,2 & 1,2 & 6 & 6 & 2,6 & 2,6 & 1,2 \\
\hline Area 7 & 5 & 5 & 6 & 6 & 6 & 5 & 5 \\
\hline Area 8 & 2 & 2 & 2 & 2 & 2 & 2 & 2 \\
\hline Area 9 & 2 & 2 & 4 & 4 & 2 & 2 & 2 \\
\hline Area 10 & 2 & 2 & 2 & 2 & 2 & 2 & 2 \\
\hline Area 11 & 3 & 3 & 3 & 3 & 3 & 3 & 3 \\
\hline Area 12 & 7 & 7 & 7 & 7 & 7 & 7 & 7 \\
\hline Area 13 & 7 & 7 & 7 & 7 & 4,7 & 7 & 7 \\
\hline Area 14 & 1 & 1 & 1 & 1 & 1 & 1 & 1 \\
\hline Area 15 & 3 & 3 & 4 & 4 & 3 & 3 & 3 \\
\hline Area 16 & 3 & 3 & 4 & 4 & 4 & 3,4 & 3,4 \\
\hline
\end{tabular}


Table 21. Distance (miles) for multi-objective patient allocation model

\begin{tabular}{|c|c|c|}
\hline Total Distance & Average Distance & Maximum Distance \\
\hline 1346.65 & 4.04 & 8.50 \\
\hline
\end{tabular}

It is noticed in Table 20, as compared to the results in Table 18, that patients from area 7 are assigned to hospitals 5 and 6 instead of hospital 1, and patients from area 14 are only assigned to hospital 1 . The multi-objective model reduces the maximum patient travel distance from 11.37 miles to 8.50 miles while the total distance is increased from 1325.03 miles to 1346.65 miles (average patient travel distance increased from 3.98 miles to 4.04 miles). The multi-objective approach results in a more equitable travel distance among individual patients.

Continue to solve the models for the second week; resource shortage (cumulative shortage for all hospitals) would occur as shown in Table 7 in section 4.1.2. Then assuming that additional resources ( 94 non-ICU beds and 7 ventilators) become available based on the resource shortage prediction, and run the models again to compare how single-objective and multi-objective models generate resource allocation and corresponding patient allocation.

To solve the single-objective optimization model, it gives the results that total travel distance is 1853.71 miles and maximum patient travel distance is 9.38 miles from patient type 3 from area 6 assigned to hospital 4.

To solve the multi-objective optimization model with the same amount of additional resources (i.e. 94 non-ICU beds and 7 ventilators), and the distance threshold still is 9 
miles, then it would not give a feasible solution, at least one patient has to be assigned to a hospital more than 9 miles away.

If the additional ventilator quantity is increased by 1 to 8 ventilators, it would obtain a feasible solution with a total travel distance of 1855.90 miles and maximum patient travel distance of 8.50 miles from patient type 1 from area 16 assigned to hospital 4. Compared to single-objective model, it gets a slightly higher total travel distance (from 1853.71 to 1855.90) and lower maximum travel distance (from 9.38 to 8.5 ), but the price is that one more ventilator is needed.

If keeping the quantities of additional resources the same (94 non-ICU beds and 7 ventilators), and increasing the distance threshold from 9 miles to 9.5 miles, it would obtain a result with a total travel distance of 1856.69 miles and maximum travel distance as 9.38 miles from patient type 3 from area 6 assigned to hospital 4 . Compared to the single-objective model, the total travel distance is increased from 1853.71 to 1855.90 miles, while the maximum travel distance is still the same 9.38 miles, which is a worse solution.

The users can perform a what-if analysis by changing the upper limit for distance threshold, as demonstrated above, to see how patients can be better served. 


\section{CHAPTER 6 CONCLUSIONS AND FUTURE STUDIES}

\subsection{Conclusions}

Pandemic influenza has been an important public health concern, considering recent incidents of $\mathrm{H} 1 \mathrm{~N} 1$ and the pandemic influenza cases in history. It is important to prepare healthcare response plans to react to a pandemic influenza outbreak. Such plans require collaboration among hospitals both in planning and in response. This dissertation focuses on patient and resource allocation among healthcare facilities in a healthcare network during a pandemic influenza outbreak. Previous work related to patient allocation mostly focuses on either long term planning (e.g. hospital network planning over a span of years) or short term planning (e.g., emergency disaster response, such as earthquake or hurricane, in a matter of hours or days). Since a pandemic outbreak usually lasts several months, it is considered to be a medium term planning problem. In this dissertation, several optimization models are formulated and solved to help decision makers address the patient and resource allocation issues faced by a multi-facility healthcare network in a medium term influenza outbreak.

Both single-objective and multi-objective optimization models are developed to help determine the patient allocation and resource allocation among healthcare facilities. The single-objective optimization models are developed to optimize the patient allocation in terms of minimizing the travel distance between patients and healthcare facilities while 
considering medical resource capacity constraints. The models also allow the decision makers to plan the allocation of patients over multiple planning horizons. During the pandemic development, the surge demand most likely would exhaust all the medical resources, at which time the models can help predict the potential resource shortage so an appropriate contingency plan can be developed. If additional quantities of resource become available, the models help to determine the best allocation of these resources among healthcare facilities. Different methods are proposed to conduct the sensitivity analysis to help decision makers determine the impact of different levels of each type of resource on the patient service. For the multi-objective optimization model it not only considers the objective of minimization of the total travel distance by patients to healthcare facilities, but also considers the balancing of the distribution of patients among healthcare facilities. A case study from Metro Louisville, KY is presented to demonstrate how the models would aid in patient allocation and resource during a pandemic influenza outbreak. A web-based application is implemented to apply the model for the decision makers. The users are able to change the values for input assumptions to do what-if analysis.

This dissertation focuses on the medium-term planning during pandemic influenza outbreak, which is different from the long-term or short-term planning considered in most literature, in the following ways:

- The planning horizon can be divided into several shorter planning horizons to reduce the solution run time and to allow the interjection of real changes in the system during the disease spread such as surge capacity increase and healthcare 
personnel infection during the pandemic development. Most current research models utilize a single planning horizon;

- The model considers the length of stay of the patients in hospitals or other alternative healthcare facilities, which is included explicitly in the capacity constraint, while most current research models simplify the stay of length by estimating a general capacity rate, such as number of patients per time unit (e.g. per year, per month).

- Sensitivity analysis is applied to optimization models in order to determine how different levels of additional resource impact the service to the patients;

- A multi-objective optimization model is developed to consider two objectives related to patients' cost of access to healthcare services, including minimization of the total travel distance by patients to service and balancing of the distribution of patients among hospitals.

\subsection{Future Studies}

The case study presented in this dissertation considered a network of several hospitals in Metro Louisville, Kentucky. Future studies will consider a system of various healthcare facilities in a state-wide or more generalized area.

FluSurge is used to generate the patient demand for this study. Future studies can include the following aspects regarding relating to the prediction of patient demand:

- Demand estimations can be generated from an improved disease spread model that considers more effects, such as social networking, and public policy with respect to mitigating the spread of influenza etc. 
- Not only the outside demand but also the inside demand can be included into the model. Inside demand represents the patients who progress from one patient type to another, such as a patient who requires an ICU bed at first then is transferred to a non-ICU bed later.

- The demand also considers the non-admitted patients as well as the admitted patients.

- The stochastic nature of the patient demand also can be included.

In this study, the capacity of the healthcare facilities is determined from a state-wide website WebEOC and only hospitals are considered in the case study. In future studies, the following can be considered regarding to the medical resource capacity:

- Future research efforts include considering healthcare facilities other than hospitals, such as clinics and urgent care centers, in the model. This modification requires no changes to the model formulations, however acquiring the input information for each additional healthcare facility may be difficult to obtain.

- In this research, the resources at each healthcare facility are not transferable, and only allow allocating the additional resources among the facilities. Future study can consider how resource transfer among facilities affects the patient service. In addition, the functional transfer between resources can be another future study, such as the conversion of an ICU bed to a non-ICU bed.

- Future studies can consider not only current facilities, but also evaluate the impact of opening new facilities.

- In this research, when patients encounter resource shortages, they are rejected by the facilities. However, in reality, they can be delayed to be admitted to the 
healthcare facilities, which can be included in future study. In this case the objective may be minimizing the delay time. 


\section{REFERENCES}

Aaby, K., Abbey, R. L., Herrmann, J. W., Treadwell, M., Jordan, C. S., \& Wood, K. (2006). Embracing computer modeling to address pandemic influenza in the $21 \mathrm{st}$ century. Journal of Public Health Management and Practice, 12(4), 365-372.

Aaby, K., Herrmann, J. W., Jordan, C. S., Treadwell, M., \& Wood, K. (2006). Montgomery County's public health service uses operations research to plan emergency mass dispensing and vaccination clinics. Interfaces, 36(6), 569-579.

Blake, J. T., \& Carter, M. W. (2002). A goal programming approach to strategic resource allocation in acute care hospitals. European Journal of Operational Research, $140,541-561$.

Bobashev, G. V., Goedecke, D. M., Yu, F., \& Epstein, J. M. (2007). A hybrid epidemic model: combining the advantages of agent-based and equation-based approaches. Proceedings of the 39th Winter Simulation Conference, 1532-1537.

Chowell, G., Ammon, C. E., Hengartner, N. W., \& Hyman, J. M. (2006). Transmission dynamics of the great influenza pandemic of 1918 in Geneva, Switzerland: Assessing the effects of hypothetical interventions. Journal of Theoretical Biology, 241(2), 193-204.

Chu, S. C. K., \& Chu, L. (2000). A modeling framework for hospital location and service allocation. International Transactions in Operational Research, 7, 539-568.

Coello, C. A. (2000). An updated survey of GA-based multiobjective optimization techniques. ACM Comput. Surv., 32(2), 109-143.

Colizza, V., Barrat, A., Barthelemy, M., Valleron, A. J., \& Vespignani, A. (2007). Modeling the worldwide spread of pandemic influenza: Baseline case and containment interventions. PLoS Medicine, 4(1), 95-110.

Das, T. K., Savachkin, A. A., \& Zhu, Y. (2008). A large-scale simulation model of pandemic influenza outbreaks for development of dynamic mitigation strategies. IIE Transactions, 40(9), 893-905.

Deb, K. (2001). Multi-objective optimization using evolutionary algorithms. Chichester: Wiley.

Ekici, A., P. Keskinocak, \& Swann, J. (2008). Pandemic influenza response. Proceedings of the 40th Winter Simulation Conference, 1592-1600.

Eubank, S., Guclu, H., Kumar, V. S. A., Marathe, M. V., Srinivasan, A., Toroczkai, Z., \& Wang, N. (2004). Modelling disease outbreaks in realistic urban social networks. Nature, 429(6988), 180-184.

Ferguson, N. M., Cummings, D. A. T., Cauchemez, S., Fraser, C., Riley, S., Meeyai, A., Iamsirithaworn, S., \& Burke, D. (2005). Strategies for containing an emerging influenza pandemic in Southeast Asia. Nature, 437(7056), 209-214.

Fiedrich, F., Gehbauer, F., \& Rickers, U. (2000). Optimized resource allocation for emergency response after earthquake disasters. Safety Science, 35, 41-57. 
Flessa, S. (2003). Priorities and allocation of health care resources in developing countries: A case-study from the Mtwara region, Tanzania. [Proceedings Paper]. European Journal of Operational Research, 150(1), 67-80.

Germann, T. C., Kadau, K., Longini Jr., I. M., \& Macken, C. A. (2006). Mitigation strategies for pandemic influenza in the United States. Proceedings of the National Academy of Sciences of the United States of America, 103(15), 59355940.

Goedecke, D. M., Bobashev, G. V., \& Yu, F. (2007). A stochastic equation-based model of the value of international air-travel restrictions for controlling pandemic flu. Proceedings of the 39th Winter Simulation Conference, 1538-1542.

Govind, R., Chatterjee, R., \& Mittal, V. (2008). Timely access to health care: Customerfocused resource allocation in a hospital network. Intern. J. of Research in Marketing, 25, 294-300.

Green, M. B., Cromley, R. G., \& Semple, R. K. (1980). The Bounded Transportation Problem. Economic Geography, 56(1), 30-44.

Gunes, E. D., \& Yaman, H. (2010). Health network mergers and hospital re-planning. Journal of the Operational Research Society, 61(2), 275-283.

Halloran, M. E., Ferguson, N. M., Eubank, S., Longini Jr., I. M., Cummings, D. A. T., Lewis, B., Xu, S., Fraser, C., Vullikanti, A., Germann, T., Wagener, D., Beckman, R., Kadau, K., Barrett, C. Macken, C., Burke, D., \& Cooley, P. (2008). Modeling targeted layered containment of an influenza pandemic in the United States. Proceedings of the National Academy of Sciences, 105(12), 4639-4644.

Harper, P. R., Shahani, A. K., Gallagher, J. E., \& Bowie, C. (2005). Planning health services with explicit geographical considerations: a stochastic location-allocation approach. Omega, 33(2), 141-152.

Holland, J. H. (1975). Adaptation in natural and artificial systems. Ann Arbor: University of Michigan Press.

Jenvald, J., Morin, M., Timpka, T., \& Eriksson, H. (2007). Simulation as decision support in pandemic influenza preparedness and response. Proceedings of 2007 International Conference on Information Systems for Crisis Response and Management, 295-304

Jones, D. F., Mirrazavi, S. K., \& Tamiz, M. (2002). Multi-objective meta-heuristics: An overview of the current state-of-the-art. [doi: DOI: 10.1016/S03772217(01)00123-0]. European Journal of Operational Research, 137(1), 1-9.

Kaplan, E. H., Craft, D. L., \& Wein, L. M. (2002). Emergency response to a smallpox attack: the case for mass vaccination. Proceedings of the National Academy of Sciences of the United States of America, 99(16), 10935-10940.

Kelley, R., Gupta, A., Kumar, A., \& Heragu, S. (2011). Using UICDS to share data in the real-time decision support system for pandemic response. 1st International Conference on Healthcare Systems Engineering, Beijing, China.

Konak, A., Coit, D. W., \& Smith, A. E. (2006). Multi-objective optimization using genetic algorithms: A tutorial. Reliability Engineering \& System Safety, 91(9), 992-1007.

Koyuncu, M., \& Erol, R. (2010). Optimal Resource Allocation Model to Mitigate the Impact of Pandemic Influenza: A Case Study for Turkey. Journal of Medical Systems, 34(1), 61-70. 
Lant, T., Jehn, M., Christensen, C., Araz, O. M., \& Fowler, J. W. (2008). Simulating pandemic influenza preparedness plans for a public university: a hierarchical system dynamics approach. Proceedings of the 40th Winter Simulation Conference, 1305-1313.

Larson, R. C. (2007). Simple models of influenza progression within a heterogeneous population. Operations research, 55(3), 399-412.

Lee, E. K., Chen, C.-H., Pietz, F., \& Benecke, B. (2009). Modeling and Optimizing the Public-Health Infrastructure for Emergency Response. Interfaces, 39(5), 476-490.

Lee, E. K., Maheshwary, S., Mason, J., \& Glisson, W. (2006a). Decision support system for mass dispensing of medications for infectious disease outbreaks and bioterrorist attacks. Annals of Operations Research, 148(1), 25-53.

Lee, E. K., Maheshwary, S., Mason, J., \& Glisson, W. (2006b). Large-scale dispensing for emergency response to bioterrorism and infectious-disease outbreak. Interfaces, 36(6), 591-607.

Lee, E. K., Smalley, H. K., Zhang, Y., Pietz, F., \& Benecke, B. (2009). Facility location and multi-modality mass dispensing strategies and emergency response for biodefence and infectious disease outbreaks. International Journal of Risk Assessment and Management, 12(2), 311-351.

Li, X., Beullens, P., Jones, D., \& Tamiz, M. (2009). An integrated queuing and multiobjective bed allocation model with application to a hospital in China. Journal of the Operational Research Society, 60(3), 330-338.

Longini Jr., I. M., Nizam, A., Xu, S., Ungchusak, K., Hanshaoworakul, W., Cummings, D., \& Halloran, M. (2005). Containing pandemic influenza at the source. Science, 309(5737), 1083-1087.

Lum, M. E., McMillan, A. J., Brook, C. W., Lester, R., \& Piers, L. S. (2009). Impact of pandemic (H1N1) 2009 influenza on critical care capacity in Victoria. Medical Journal of Australia, 191(9), 502-506.

Marler, R. T., \& Arora, J. S. (2004). Survey of multi-objective optimization methods for engineering. [Review]. Structural and Multidisciplinary Optimization, 26(6), 369395.

Meltzer, M. I., Cox, N. J., \& Fukuda, K. (1999). The Economic Impact of Pandemic Influenza in the United States: Priorities for Intervention. Emerging Infectious Diseases, 5(5), 659-671.

Meltzer, M. I., Shoemake, H. A., Kownaski, M., \& Crosby, R. (2000). FluAid 2.0: A manual to aid state and local-level public health officials plan, prepare and practice for the next influenza pandemic (Beta test version): Centers for Disease Control and Prevention, U.S. Department of Health and Human Services.

Menon, D. K., Taylor, B. L., \& Ridley, S. A. (2005). Modelling the impact of an influenza pandemic on critical care services in England. Anaesthesia, 60(10), 952954.

Minciardi, R., Sacile, R., \& Trasforini, E. (2009). Resource Allocation in Integrated Preoperational and Operational Management of Natural Hazards. Risk Analysis, 29(1), 62-75.

Mitropoulos, P., Mitropoulos, I., Giannikos, I., \& Sissouras, A. (2006). A biobjective model for the locational planning of hospitals and health centers. Health Care Management Science, 9, 171-179. 
Naron, S., \& Wasserkrug, S. (2007). Utilizing model characteristics to obtain efficient parallelization in the context of agent based epidemiological models. Proceedings of the 39th Winter Simulation Conference, 1555-1561.

Oddoye, J., Yaghoobi, M., Tamiz, M., Jones, D., \& Schmidt, P. (2007). A multi-objective model to determine efficient resource levels in a medical assessment unit. The Journal of the Operational Research Society, 58(12), 1563.

Parker, J. (2007). A flexible, large-scale, distributed agent based epidemic model. Proceedings of the 39th Winter Simulation Conference, 1543-1547.

Rico, F., Salari, E., \& Centeno, G. (2007). Emergency departments nurse allocation to face a pandemic influenza outbreak. Proceedings of the 39th Winter Simulation Conference, 1292-1298.

Ruth, J. R. (1981). A Mixed Integer Programming Model for Regional Planning of a Hospital Inpatient Service. Management Science, 27(5), 521-533.

Santibanez, P., Bekiou, G., \& Yip, K. (2009). Fraser Health Uses Mathematical Programming to Plan Its Inpatient Hospital Network. Interfaces, 39(3), 196-208.

Schweikhart, S. B., \& Smith-Daniels, V. L. (1993). Location and Service Mix Decisions for a Managed Health Care Network. Socio-Economic Planning Sciences, 27(4), 289-302.

Sobieraj, J. A., Reyes, J., Dunemn, K. N., Carty, I. H., Pennathur, A., Gutierrez, R. S., \& Harris, M. (2007). Modeling hospital response to mild and severe influenza pandemic scenarios under normal and expanded capacities. Military medicine, 172(5), 486-490.

Stummer, C., Doerner, K., Focke, A., \& Heidenberger, K. (2004). Determining Location and Size of Medical Departments in a Hospital Network: A Multiobjective Decision Support Approach. Health Care Management Science, 7(1), 63-71.

Suman, B., \& Kumar, P. (2006). A Survey of Simulated Annealing as a Tool for Single and Multiobjective Optimization. The Journal of the Operational Research Society, 57(10), 1143-1160.

Syam, S. S., \& Côté, M. J. (2010). A location-allocation model for service providers with application to not-for-profit health care organizations. Omega, 38(3-4), 157-166.

Toner, E., \& Waldhorn, R. (2006). What hospitals should do to prepare for an influenza pandemic. Biosecurity and Bioterrorism: Biodefense Strategy, Practice, and Science, 4(4), 397-402.

Ward, J. E., \& Wendell, R. E. (1990). Approaches to sensitivity analysis in linear programming. Annals of Operations Research, 27(1), 3-38.

Wilson, N., Mansoor, O., \& Baker, M. (2005). Estimating the impact of the next influenza pandemic on population health and health sector capacity in New Zealand. The New Zealand medical journal, 118(1211), U1346.

Wu, J. T., Riley, S., Fraser, C., \& Leung, G. M. (2006). Reducing the Impact of the Next Influenza Pandemic Using Household-Based Public Health Interventions. PLoS Medicine, 3(9), 1532-1540.

Yi, W., \& Ozdamar, L. (2007). A dynamic logistics coordination model for evacuation and support in disaster response activities. [Proceedings Paper]. European Journal of Operational Research, 179(3), 1177-1193.

Zaric, G. S., \& Brandeau, M. L. (2001). Resource allocation for epidemic control over short time horizons. Mathematical Biosciences, 171, 33-58. 
Zhang, X., Meltzer, M. I., \& Wortley, P. (2005). FluSurge2. 0: a manual to assist state and local public health officials and hospital administrators in estimating the impact of an influenza pandemic on hospital surge capacity (Beta test version): Centers for Disease Control and Prevention, U.S. Department of Health and Human Services.

Zhang, X., Meltzer, M. I., \& Wortley, P. (2006). FluSurge--a tool to estimate demand for hospital services during the next pandemic influenza. Medical Decision Making, 26(6), 617-623. 


\section{APPENDIX}

Table 22. Demand estimation for 6-week pandemic duration, $35 \%$ gross attack rate, most likely scenario (day 1- 14)

\begin{tabular}{|c|c|c|c|c|c|c|c|c|c|c|c|c|c|c|c|}
\hline \multicolumn{2}{|c|}{ Demand } & D1 & D2 & D3 & D4 & D5 & D6 & D7 & D8 & D9 & D10 & D11 & D12 & D13 & D14 \\
\hline \multirow{16}{*}{ P1 } & A1 & 1 & 1 & 1 & 1 & 2 & 2 & 2 & 2 & 2 & 2 & 2 & 2 & 2 & 2 \\
\hline & $\mathrm{A} 2$ & 2 & 2 & 2 & 2 & 2 & 2 & 2 & 3 & 3 & 3 & 3 & 3 & 3 & 4 \\
\hline & $\mathrm{A3}$ & 1 & 1 & 2 & 2 & 2 & 2 & 2 & 2 & 2 & 2 & 2 & 2 & 2 & 2 \\
\hline & A4 & 2 & 2 & 2 & 3 & 3 & 3 & 3 & 3 & 3 & 4 & 4 & 4 & 4 & 4 \\
\hline & A5 & 4 & 4 & 4 & 4 & 4 & 4 & 4 & 5 & 5 & 5 & 6 & 6 & 6 & 6 \\
\hline & A6 & 5 & 5 & 5 & 6 & 6 & 6 & 6 & 7 & 7 & 8 & 8 & 8 & 8 & 9 \\
\hline & A7 & 2 & 2 & 2 & 2 & 2 & 3 & 3 & 3 & 3 & 3 & 3 & 3 & 4 & 4 \\
\hline & A8 & 2 & 2 & 2 & 2 & 2 & 2 & 2 & 3 & 3 & 3 & 3 & 3 & 3 & 3 \\
\hline & A9 & 2 & 2 & 2 & 2 & 2 & 2 & 2 & 3 & 3 & 3 & 3 & 3 & 3 & 3 \\
\hline & A10 & 1 & 2 & 2 & 2 & 2 & 2 & 2 & 2 & 2 & 2 & 2 & 2 & 2 & 3 \\
\hline & $\mathrm{A} 11$ & 4 & 4 & 4 & 4 & 4 & 4 & 4 & 5 & 5 & 6 & 6 & 6 & 6 & 6 \\
\hline & A12 & 3 & 3 & 3 & 3 & 3 & 3 & 3 & 4 & 4 & 4 & 4 & 4 & 4 & 4 \\
\hline & A13 & 3 & 3 & 3 & 3 & 3 & 3 & 3 & 4 & 4 & 4 & 4 & 4 & 5 & 5 \\
\hline & A14 & 4 & 4 & 4 & 4 & 5 & 5 & 5 & 6 & 6 & 6 & 6 & 7 & 7 & 7 \\
\hline & A15 & 3 & 3 & 3 & 3 & 3 & 4 & 4 & 4 & 4 & 5 & 5 & 5 & 5 & 5 \\
\hline & A16 & 3 & 3 & 3 & 4 & 4 & 4 & 4 & 5 & 5 & 5 & 5 & 5 & 5 & 6 \\
\hline \multirow{16}{*}{ P2 } & $\mathrm{A} 1$ & 0 & 0 & 0 & 0 & 0 & 0 & 0 & 0 & 0 & 0 & 0 & 0 & 0 & 0 \\
\hline & $\mathrm{A} 2$ & 0 & 0 & 0 & 0 & 0 & 0 & 0 & 0 & 0 & 0 & 0 & 0 & 0 & 0 \\
\hline & $\mathrm{A3}$ & 0 & 0 & 0 & 0 & 0 & 0 & 0 & 0 & 0 & 0 & 0 & 0 & 0 & 0 \\
\hline & A4 & 0 & 0 & 0 & 0 & 0 & 0 & 0 & 0 & 0 & 0 & 0 & 0 & 0 & 0 \\
\hline & A5 & 0 & 0 & 0 & 0 & 0 & 0 & 0 & 0 & 0 & 0 & 0 & 1 & 1 & 1 \\
\hline & A6 & 0 & 0 & 0 & 0 & 1 & 1 & 1 & 1 & 1 & 1 & 1 & 1 & 1 & 1 \\
\hline & A7 & 0 & 0 & 0 & 0 & 0 & 0 & 0 & 0 & 0 & 0 & 0 & 0 & 0 & 0 \\
\hline & A8 & 0 & 0 & 0 & 0 & 0 & 0 & 0 & 0 & 0 & 0 & 0 & 0 & 0 & 0 \\
\hline & A9 & 0 & 0 & 0 & 0 & 0 & 0 & 0 & 0 & 0 & 0 & 0 & 0 & 0 & 0 \\
\hline & A10 & 0 & 0 & 0 & 0 & 0 & 0 & 0 & 0 & 0 & 0 & 0 & 0 & 0 & 0 \\
\hline & A11 & 0 & 0 & 0 & 0 & 0 & 0 & 0 & 0 & 0 & 0 & 1 & 1 & 1 & 1 \\
\hline & $\mathrm{A} 12$ & 0 & 0 & 0 & 0 & 0 & 0 & 0 & 0 & 0 & 0 & 0 & 0 & 0 & 0 \\
\hline & A13 & 0 & 0 & 0 & 0 & 0 & 0 & 0 & 0 & 0 & 0 & 0 & 0 & 0 & 0 \\
\hline & A14 & 0 & 0 & $\overline{0}$ & 0 & 0 & 0 & 0 & 1 & 1 & 1 & 1 & 1 & 1 & 1 \\
\hline & A15 & 0 & 0 & 0 & 0 & 0 & 0 & 0 & 0 & 0 & 0 & 0 & 0 & 0 & 0 \\
\hline & A16 & 0 & 0 & 0 & 0 & 0 & 0 & 0 & 0 & 0 & 0 & 0 & 0 & 0 & 0 \\
\hline \multirow{4}{*}{ P3 } & $\mathrm{A} 1$ & 0 & 0 & 0 & 0 & 0 & 0 & 0 & 0 & 0 & 0 & 0 & 0 & 0 & 0 \\
\hline & A2 & 0 & 0 & 0 & 0 & 0 & 0 & 0 & 0 & 0 & 0 & 0 & 0 & 0 & 0 \\
\hline & A3 & 0 & 0 & 0 & 0 & 0 & 0 & 0 & 0 & 0 & 0 & 0 & 0 & 0 & 0 \\
\hline & A4 & 0 & 0 & 0 & 0 & 0 & 0 & 0 & 0 & 0 & 0 & 0 & 0 & 0 & 0 \\
\hline
\end{tabular}




\begin{tabular}{|c|c|c|c|c|c|c|c|c|c|c|c|c|c|c|c|}
\hline A5 & 0 & 0 & 0 & 0 & 0 & 0 & 0 & 0 & 0 & 0 & 0 & 1 & 1 & 1 \\
\hline A6 & 0 & 0 & 0 & 0 & 1 & 1 & 1 & 1 & 1 & 1 & 1 & 1 & 1 & 1 \\
\hline A7 & 0 & 0 & 0 & 0 & 0 & 0 & 0 & 0 & 0 & 0 & 0 & 0 & 0 & 0 \\
\hline A8 & 0 & 0 & 0 & 0 & 0 & 0 & 0 & 0 & 0 & 0 & 0 & 0 & 0 & 0 \\
\hline A9 & 0 & 0 & 0 & 0 & 0 & 0 & 0 & 0 & 0 & 0 & 0 & 0 & 0 & 0 \\
\hline A10 & 0 & 0 & 0 & 0 & 0 & 0 & 0 & 0 & 0 & 0 & 0 & 0 & 0 & 0 \\
\hline A11 & 0 & 0 & 0 & 0 & 0 & 0 & 0 & 0 & 0 & 0 & 1 & 1 & 1 & 1 \\
\hline A12 & 0 & 0 & 0 & 0 & 0 & 0 & 0 & 0 & 0 & 0 & 0 & 0 & 0 & 0 \\
\hline A13 & 0 & 0 & 0 & 0 & 0 & 0 & 0 & 0 & 0 & 0 & 0 & 0 & 0 & 0 \\
\hline A14 & 0 & 0 & 0 & 0 & 0 & 0 & 0 & 1 & 1 & 1 & 1 & 1 & 1 & 1 \\
\hline A15 & 0 & 0 & 0 & 0 & 0 & 0 & 0 & 0 & 0 & 0 & 0 & 0 & 0 & 0 \\
\hline A16 & 0 & 0 & 0 & 0 & 0 & 0 & 0 & 0 & 0 & 0 & 0 & 0 & 0 & 0 \\
\hline
\end{tabular}

Table 23. Demand estimation for 6-week pandemic duration, 35\% gross attack rate, most likely scenario (day 15- 28)

\begin{tabular}{|c|c|c|c|c|c|c|c|c|c|c|c|c|c|c|c|}
\hline \multicolumn{2}{|c|}{ Demand } & D15 & D16 & D17 & D18 & D19 & D20 & D21 & D22 & D23 & D24 & D25 & D26 & D27 & D28 \\
\hline A1 & 2 & 2 & 2 & 3 & 3 & 3 & 3 & 3 & 3 & 3 & 3 & 2 & 2 & 2 \\
\hline A2 & 4 & 4 & 4 & 4 & 4 & 4 & 4 & 4 & 4 & 4 & 4 & 4 & 4 & 4 \\
\hline A3 & 3 & 3 & 3 & 3 & 3 & 3 & 3 & 3 & 3 & 3 & 3 & 3 & 3 & 3 \\
\hline A4 & 4 & 4 & 4 & 4 & 5 & 5 & 5 & 5 & 5 & 5 & 4 & 4 & 4 & 4 \\
\hline A5 & 6 & 6 & 7 & 7 & 7 & 7 & 8 & 8 & 7 & 7 & 7 & 7 & 6 & 6 \\
\hline A6 & 9 & 9 & 9 & 10 & 10 & 10 & 11 & 11 & 10 & 10 & 10 & 9 & 9 & 9 \\
\hline A7 & 4 & 4 & 4 & 4 & 4 & 4 & 5 & 5 & 4 & 4 & 4 & 4 & 4 & 4 \\
\hline A8 & 3 & 3 & 3 & 4 & 4 & 4 & 4 & 4 & 4 & 4 & 4 & 3 & 3 & 3 \\
\hline A9 & 3 & 4 & 4 & 4 & 4 & 4 & 4 & 4 & 4 & 4 & 4 & 4 & 4 & 3 \\
\hline A10 & 3 & 3 & 3 & 3 & 3 & 3 & 3 & 3 & 3 & 3 & 3 & 3 & 3 & 3 \\
\hline A11 & 7 & 7 & 7 & 7 & 7 & 8 & 8 & 8 & 8 & 7 & 7 & 7 & 7 & 7 \\
\hline A12 & 4 & 5 & 5 & 5 & 5 & 5 & 5 & 5 & 5 & 5 & 5 & 5 & 5 & 4 \\
\hline A13 & 5 & 5 & 5 & 5 & 6 & 6 & 6 & 6 & 6 & 6 & 5 & 5 & 5 & 5 \\
\hline A14 & 7 & 7 & 8 & 8 & 8 & 8 & 9 & 9 & 8 & 8 & 8 & 8 & 7 & 7 \\
\hline A15 & 5 & 5 & 6 & 6 & 6 & 6 & 6 & 6 & 6 & 6 & 6 & 6 & 5 & 5 \\
\hline A16 & 6 & 6 & 6 & 6 & 6 & 7 & 7 & 7 & 7 & 6 & 6 & 6 & 6 & 6 \\
\hline A1 & 0 & 0 & 0 & 0 & 0 & 0 & 0 & 0 & 0 & 0 & 0 & 0 & 0 & 0 \\
\hline A2 & 0 & 0 & 0 & 0 & 0 & 0 & 0 & 0 & 0 & 0 & 0 & 0 & 0 & 0 \\
\hline A3 & 0 & 0 & 0 & 0 & 0 & 0 & 0 & 0 & 0 & 0 & 0 & 0 & 0 & 0 \\
\hline A4 & 0 & 0 & 0 & 0 & 0 & 0 & 0 & 0 & 0 & 0 & 0 & 0 & 0 & 0 \\
\hline A5 & 1 & 1 & 1 & 1 & 1 & 1 & 1 & 1 & 1 & 1 & 1 & 1 & 1 & 1 \\
\hline A6 & 1 & 1 & 1 & 1 & 1 & 1 & 1 & 1 & 1 & 1 & 1 & 1 & 1 & 1 \\
\hline A7 & 0 & 0 & 0 & 0 & 0 & 0 & 0 & 0 & 0 & 0 & 0 & 0 & 0 & 0 \\
\hline A8 & 0 & 0 & 0 & 0 & 0 & 0 & 0 & 0 & 0 & 0 & 0 & 0 & 0 & 0 \\
\hline A9 & 0 & 0 & 0 & 0 & 0 & 0 & 0 & 0 & 0 & 0 & 0 & 0 & 0 & 0 \\
\hline A10 & 0 & 0 & 0 & 0 & 0 & 0 & 0 & 0 & 0 & 0 & 0 & 0 & 0 & 0 \\
\hline A11 & 1 & 1 & 1 & 1 & 1 & 1 & 1 & 1 & 1 & 1 & 1 & 1 & 1 & 1 \\
\hline A12 & 0 & 0 & 0 & 0 & 0 & 0 & 0 & 0 & 0 & 0 & 0 & 0 & 0 & 0 \\
\hline A13 & 0 & 0 & 0 & 0 & 0 & 1 & 1 & 1 & 1 & 0 & 0 & 0 & 0 & 0 \\
\hline A14 & 1 & 1 & 1 & 1 & 1 & 1 & 1 & 1 & 1 & 1 & 1 & 1 & 1 & 1 \\
\hline
\end{tabular}




\begin{tabular}{|c|c|c|c|c|c|c|c|c|c|c|c|c|c|c|c|}
\hline A15 & 0 & 0 & 0 & 1 & 1 & 1 & 1 & 1 & 1 & 1 & 1 & 0 & 0 & 0 \\
\hline A16 & 1 & 1 & 1 & 1 & 1 & 1 & 1 & 1 & 1 & 1 & 1 & 1 & 1 & 1 \\
\hline A1 & 0 & 0 & 0 & 0 & 0 & 0 & 0 & 0 & 0 & 0 & 0 & 0 & 0 & 0 \\
\hline A2 & 0 & 0 & 0 & 0 & 0 & 0 & 0 & 0 & 0 & 0 & 0 & 0 & 0 & 0 \\
\hline A3 & 0 & 0 & 0 & 0 & 0 & 0 & 0 & 0 & 0 & 0 & 0 & 0 & 0 & 0 \\
\hline A4 & 0 & 0 & 0 & 0 & 0 & 0 & 0 & 0 & 0 & 0 & 0 & 0 & 0 & 0 \\
\hline A5 & 1 & 1 & 1 & 1 & 1 & 1 & 1 & 1 & 1 & 1 & 1 & 1 & 1 & 1 \\
\hline A6 & 1 & 1 & 1 & 1 & 1 & 1 & 1 & 1 & 1 & 1 & 1 & 1 & 1 & 1 \\
\hline A7 & 0 & 0 & 0 & 0 & 0 & 0 & 0 & 0 & 0 & 0 & 0 & 0 & 0 & 0 \\
\hline A8 & 0 & 0 & 0 & 0 & 0 & 0 & 0 & 0 & 0 & 0 & 0 & 0 & 0 & 0 \\
\hline A9 & 0 & 0 & 0 & 0 & 0 & 0 & 0 & 0 & 0 & 0 & 0 & 0 & 0 & 0 \\
\hline A10 & 0 & 0 & 0 & 0 & 0 & 0 & 0 & 0 & 0 & 0 & 0 & 0 & 0 & 0 \\
\hline A11 & 1 & 1 & 1 & 1 & 1 & 1 & 1 & 1 & 1 & 1 & 1 & 1 & 1 & 1 \\
\hline A12 & 0 & 0 & 0 & 0 & 0 & 0 & 0 & 0 & 0 & 0 & 0 & 0 & 0 & 0 \\
\hline A13 & 0 & 0 & 0 & 0 & 0 & 1 & 1 & 1 & 1 & 0 & 0 & 0 & 0 & 0 \\
\hline A14 & 1 & 1 & 1 & 1 & 1 & 1 & 1 & 1 & 1 & 1 & 1 & 1 & 1 & 1 \\
\hline A15 & 0 & 0 & 0 & 1 & 1 & 1 & 1 & 1 & 1 & 1 & 1 & 0 & 0 & 0 \\
\hline A16 & 1 & 1 & 1 & 1 & 1 & 1 & 1 & 1 & 1 & 1 & 1 & 1 & 1 & 1 \\
\hline
\end{tabular}

Table 24. Demand estimation for 6-week pandemic duration, 35\% gross attack rate, most likely scenario (day 29-42)

\begin{tabular}{|l|c|c|c|c|c|c|c|c|c|c|c|c|c|c|c|}
\hline Demand & D29 & D30 & D31 & D32 & D33 & D34 & D35 & D36 & D37 & D38 & D39 & D40 & D41 & D42 \\
\hline A1 & 2 & 2 & 2 & 2 & 2 & 2 & 2 & 2 & 2 & 2 & 1 & 1 & 1 & 1 \\
\hline A2 & 4 & 3 & 3 & 3 & 3 & 3 & 3 & 2 & 2 & 2 & 2 & 2 & 2 & 2 \\
\hline A3 & 2 & 2 & 2 & 2 & 2 & 2 & 2 & 2 & 2 & 2 & 2 & 2 & 1 & 1 \\
\hline A4 & 4 & 4 & 4 & 4 & 4 & 3 & 3 & 3 & 3 & 3 & 3 & 2 & 2 & 2 \\
\hline A5 & 6 & 6 & 6 & 6 & 5 & 5 & 5 & 4 & 4 & 4 & 4 & 4 & 4 & 4 \\
\hline A6 & 9 & 8 & 8 & 8 & 8 & 7 & 7 & 6 & 6 & 6 & 6 & 5 & 5 & 5 \\
\hline A7 & 4 & 4 & 3 & 3 & 3 & 3 & 3 & 3 & 3 & 2 & 2 & 2 & 2 & 2 \\
\hline A8 & 3 & 3 & 3 & 3 & 3 & 3 & 3 & 2 & 2 & 2 & 2 & 2 & 2 & 2 \\
\hline A9 & 3 & 3 & 3 & 3 & 3 & 3 & 3 & 2 & 2 & 2 & 2 & 2 & 2 & 2 \\
\hline A10 & 3 & 2 & 2 & 2 & 2 & 2 & 2 & 2 & 2 & 2 & 2 & 2 & 2 & 1 \\
\hline A11 & 6 & 6 & 6 & 6 & 6 & 5 & 5 & 4 & 4 & 4 & 4 & 4 & 4 & 4 \\
\hline A12 & 4 & 4 & 4 & 4 & 4 & 4 & 4 & 3 & 3 & 3 & 3 & 3 & 3 & 3 \\
\hline A13 & 5 & 5 & 4 & 4 & 4 & 4 & 4 & 3 & 3 & 3 & 3 & 3 & 3 & 3 \\
\hline A14 & 7 & 7 & 7 & 6 & 6 & 6 & 6 & 5 & 5 & 5 & 4 & 4 & 4 & 4 \\
\hline A15 & 5 & 5 & 5 & 5 & 5 & 4 & 4 & 4 & 4 & 3 & 3 & 3 & 3 & 3 \\
\hline A16 & 6 & 5 & 5 & 5 & 5 & 5 & 5 & 4 & 4 & 4 & 4 & 3 & 3 & 3 \\
\hline A1 & 0 & 0 & 0 & 0 & 0 & 0 & 0 & 0 & 0 & 0 & 0 & 0 & 0 & 0 \\
\hline A2 & 0 & 0 & 0 & 0 & 0 & 0 & 0 & 0 & 0 & 0 & 0 & 0 & 0 & 0 \\
\hline A3 & 0 & 0 & 0 & 0 & 0 & 0 & 0 & 0 & 0 & 0 & 0 & 0 & 0 & 0 \\
\hline A4 & 0 & 0 & 0 & 0 & 0 & 0 & 0 & 0 & 0 & 0 & 0 & 0 & 0 & 0 \\
\hline A5 & 1 & 1 & 1 & 0 & 0 & 0 & 0 & 0 & 0 & 0 & 0 & 0 & 0 & 0 \\
\hline A6 & 1 & 1 & 1 & 1 & 1 & 1 & 1 & 1 & 1 & 1 & 0 & 0 & 0 & 0 \\
\hline A7 & 0 & 0 & 0 & 0 & 0 & 0 & 0 & 0 & 0 & 0 & 0 & 0 & 0 & 0 \\
\hline A8 & 0 & 0 & 0 & 0 & 0 & 0 & 0 & 0 & 0 & 0 & 0 & 0 & 0 & 0 \\
\hline A9 & 0 & 0 & 0 & 0 & 0 & 0 & 0 & 0 & 0 & 0 & 0 & 0 & 0 & 0 \\
\hline
\end{tabular}




\begin{tabular}{|c|c|c|c|c|c|c|c|c|c|c|c|c|c|c|c|}
\hline A10 & 0 & 0 & 0 & 0 & 0 & 0 & 0 & 0 & 0 & 0 & 0 & 0 & 0 & 0 \\
\hline A11 & 1 & 1 & 1 & 1 & 0 & 0 & 0 & 0 & 0 & 0 & 0 & 0 & 0 & 0 \\
\hline A12 & 0 & 0 & 0 & 0 & 0 & 0 & 0 & 0 & 0 & 0 & 0 & 0 & 0 & 0 \\
\hline A13 & 0 & 0 & 0 & 0 & 0 & 0 & 0 & 0 & 0 & 0 & 0 & 0 & 0 & 0 \\
\hline A14 & 1 & 1 & 1 & 1 & 1 & 1 & 1 & 0 & 0 & 0 & 0 & 0 & 0 & 0 \\
\hline A15 & 0 & 0 & 0 & 0 & 0 & 0 & 0 & 0 & 0 & 0 & 0 & 0 & 0 & 0 \\
\hline A16 & 0 & 0 & 0 & 0 & 0 & 0 & 0 & 0 & 0 & 0 & 0 & 0 & 0 & 0 \\
\hline A1 & 0 & 0 & 0 & 0 & 0 & 0 & 0 & 0 & 0 & 0 & 0 & 0 & 0 & 0 \\
\hline A2 & 0 & 0 & 0 & 0 & 0 & 0 & 0 & 0 & 0 & 0 & 0 & 0 & 0 & 0 \\
\hline A3 & 0 & 0 & 0 & 0 & 0 & 0 & 0 & 0 & 0 & 0 & 0 & 0 & 0 & 0 \\
\hline A4 & 0 & 0 & 0 & 0 & 0 & 0 & 0 & 0 & 0 & 0 & 0 & 0 & 0 & 0 \\
\hline A5 & 1 & 1 & 1 & 0 & 0 & 0 & 0 & 0 & 0 & 0 & 0 & 0 & 0 & 0 \\
\hline A6 & 1 & 1 & 1 & 1 & 1 & 1 & 1 & 1 & 1 & 1 & 0 & 0 & 0 & 0 \\
\hline A7 & 0 & 0 & 0 & 0 & 0 & 0 & 0 & 0 & 0 & 0 & 0 & 0 & 0 & 0 \\
\hline A8 & 0 & 0 & 0 & 0 & 0 & 0 & 0 & 0 & 0 & 0 & 0 & 0 & 0 & 0 \\
\hline A9 & 0 & 0 & 0 & 0 & 0 & 0 & 0 & 0 & 0 & 0 & 0 & 0 & 0 & 0 \\
\hline A10 & 0 & 0 & 0 & 0 & 0 & 0 & 0 & 0 & 0 & 0 & 0 & 0 & 0 & 0 \\
\hline A11 & 1 & 1 & 1 & 1 & 0 & 0 & 0 & 0 & 0 & 0 & 0 & 0 & 0 & 0 \\
\hline A12 & 0 & 0 & 0 & 0 & 0 & 0 & 0 & 0 & 0 & 0 & 0 & 0 & 0 & 0 \\
\hline A13 & 0 & 0 & 0 & 0 & 0 & 0 & 0 & 0 & 0 & 0 & 0 & 0 & 0 & 0 \\
\hline A14 & 1 & 1 & 1 & 1 & 1 & 1 & 1 & 0 & 0 & 0 & 0 & 0 & 0 & 0 \\
\hline A15 & 0 & 0 & 0 & 0 & 0 & 0 & 0 & 0 & 0 & 0 & 0 & 0 & 0 & 0 \\
\hline A16 & 0 & 0 & 0 & 0 & 0 & 0 & 0 & 0 & 0 & 0 & 0 & 0 & 0 & 0 \\
\hline
\end{tabular}

Table 25. Allocation of patient type 1 for 6-week pandemic duration, 35\% gross attack rate, most likely scenario (day 1-14)

\begin{tabular}{|c|c|c|c|c|c|c|c|c|c|c|c|c|c|c|c|}
\hline & $\mathrm{X}$ & $\mathrm{D} 1$ & $\mathrm{D} 2$ & $\mathrm{D} 3$ & $\mathrm{D} 4$ & $\mathrm{D} 5$ & $\mathrm{D} 6$ & $\mathrm{D} 7$ & $\mathrm{D} 8$ & $\mathrm{D} 9$ & $\mathrm{D} 10$ & $\mathrm{D} 11$ & $\mathrm{D} 12$ & $\mathrm{D} 13$ & $\mathrm{D} 14$ \\
\hline $\mathrm{A} 1$ & $\mathrm{H} 6$ & 1 & 1 & 1 & 1 & 2 & 2 & 2 & 2 & 2 & 2 & 2 & 2 & 2 & 2 \\
\hline $\mathrm{A} 2$ & $\mathrm{H} 4$ & 2 & 2 & 2 & 2 & 2 & 2 & 2 & 3 & 3 & 3 & 3 & 3 & 3 & 4 \\
\hline $\mathrm{A} 3$ & $\mathrm{H} 6$ & 1 & 1 & 2 & 2 & 2 & 2 & 2 & 2 & 2 & 2 & 2 & 2 & 2 & 2 \\
\hline $\mathrm{A} 4$ & $\mathrm{H} 4$ & 2 & 2 & 2 & 3 & 3 & 3 & 3 & 3 & 3 & 4 & 4 & 4 & 4 & 4 \\
\hline $\mathrm{A} 5$ & $\mathrm{H} 5$ & 4 & 4 & 4 & 4 & 2 & 4 & 4 & 5 & 5 & 2 & 6 & 6 & 6 & 6 \\
\hline $\mathrm{A} 5$ & $\mathrm{H} 6$ & 0 & 0 & 0 & 0 & 2 & 0 & 0 & 0 & 0 & 3 & 0 & 0 & 0 & 0 \\
\hline $\mathrm{A} 6$ & $\mathrm{H} 1$ & 3 & 4 & 0 & 3 & 0 & 2 & 3 & 4 & 7 & 0 & 7 & 8 & 4 & 7 \\
\hline $\mathrm{A} 6$ & $\mathrm{H} 2$ & 2 & 1 & 5 & 0 & 4 & 2 & 1 & 2 & 0 & 3 & 1 & 0 & 4 & 2 \\
\hline $\mathrm{A} 6$ & $\mathrm{H} 6$ & 0 & 0 & 0 & 3 & 2 & 2 & 2 & 1 & 0 & 5 & 0 & 0 & 0 & 0 \\
\hline $\mathrm{A} 7$ & $\mathrm{H} 5$ & 2 & 2 & 2 & 2 & 0 & 2 & 2 & 1 & 1 & 0 & 0 & 0 & 0 & 0 \\
\hline $\mathrm{A} 7$ & $\mathrm{H} 6$ & 0 & 0 & 0 & 0 & 2 & 1 & 1 & 2 & 2 & 3 & 3 & 3 & 3 & 2 \\
\hline $\mathrm{A} 7$ & $\mathrm{DH}$ & 0 & 0 & 0 & 0 & 0 & 0 & 0 & 0 & 0 & 0 & 0 & 0 & 1 & 2 \\
\hline $\mathrm{A} 8$ & $\mathrm{H} 2$ & 2 & 2 & 2 & 2 & 2 & 2 & 2 & 3 & 3 & 3 & 3 & 3 & 3 & 3 \\
\hline $\mathrm{A} 9$ & $\mathrm{H} 2$ & 2 & 2 & 1 & 2 & 0 & 1 & 2 & 3 & 1 & 0 & 1 & 2 & 1 & 1 \\
\hline $\mathrm{A} 9$ & $\mathrm{H} 3$ & 0 & 0 & 0 & 0 & 0 & 0 & 0 & 0 & 0 & 0 & 2 & 1 & 1 & 0 \\
\hline $\mathrm{A} 9$ & $\mathrm{H} 4$ & 0 & 0 & 1 & 0 & 2 & 1 & 0 & 0 & 2 & 3 & 0 & 0 & 1 & 2 \\
\hline $\mathrm{A} 10$ & $\mathrm{H} 2$ & 1 & 2 & 2 & 2 & 2 & 2 & 2 & 2 & 2 & 2 & 2 & 2 & 2 & 0 \\
\hline
\end{tabular}




\begin{tabular}{|l|l|l|l|l|l|l|l|l|l|l|l|l|l|l|l|}
\hline $\mathrm{A} 10$ & $\mathrm{DH}$ & 0 & 0 & 0 & 0 & 0 & 0 & 0 & 0 & 0 & 0 & 0 & 0 & 0 & 3 \\
\hline $\mathrm{A} 11$ & $\mathrm{H} 3$ & 4 & 4 & 4 & 4 & 2 & 4 & 4 & 5 & 5 & 2 & 6 & 6 & 6 & 6 \\
\hline $\mathrm{A} 11$ & $\mathrm{H} 4$ & 0 & 0 & 0 & 0 & 2 & 0 & 0 & 0 & 0 & 4 & 0 & 0 & 0 & 0 \\
\hline $\mathrm{A} 12$ & $\mathrm{H} 7$ & 3 & 3 & 3 & 3 & 3 & 3 & 3 & 4 & 4 & 4 & 4 & 4 & 4 & 4 \\
\hline $\mathrm{A} 13$ & $\mathrm{H} 3$ & 0 & 0 & 0 & 0 & 0 & 0 & 0 & 0 & 0 & 0 & 1 & 2 & 0 & 0 \\
\hline $\mathrm{A} 13$ & $\mathrm{H} 4$ & 0 & 0 & 0 & 0 & 2 & 0 & 0 & 2 & 2 & 4 & 1 & 0 & 0 & 0 \\
\hline $\mathrm{A} 13$ & $\mathrm{H} 7$ & 3 & 3 & 3 & 3 & 1 & 3 & 3 & 2 & 2 & 0 & 2 & 2 & 2 & 2 \\
\hline $\mathrm{A} 13$ & $\mathrm{DH}$ & 0 & 0 & 0 & 0 & 0 & 0 & 0 & 0 & 0 & 0 & 0 & 0 & 3 & 3 \\
\hline $\mathrm{A} 14$ & $\mathrm{H} 1$ & 4 & 4 & 4 & 4 & 0 & 5 & 5 & 0 & 0 & 0 & 0 & 0 & 0 & 0 \\
\hline $\mathrm{A} 14$ & $\mathrm{H} 6$ & 0 & 0 & 0 & 0 & 5 & 0 & 0 & 0 & 0 & 0 & 0 & 0 & 0 & 0 \\
\hline $\mathrm{A} 14$ & $\mathrm{DH}$ & 0 & 0 & 0 & 0 & 0 & 0 & 0 & 6 & 6 & 6 & 6 & 7 & 7 & 7 \\
\hline $\mathrm{A} 15$ & $\mathrm{H} 3$ & 3 & 3 & 3 & 2 & 0 & 4 & 4 & 2 & 1 & 0 & 1 & 1 & 0 & 0 \\
\hline $\mathrm{A} 15$ & $\mathrm{H} 4$ & 0 & 0 & 0 & 1 & 3 & 0 & 0 & 0 & 0 & 0 & 0 & 0 & 0 & 0 \\
\hline $\mathrm{A} 15$ & $\mathrm{DH}$ & 0 & 0 & 0 & 0 & 0 & 0 & 0 & 2 & 3 & 5 & 4 & 4 & 5 & 5 \\
\hline $\mathrm{A} 16$ & $\mathrm{H} 3$ & 3 & 3 & 0 & 0 & 0 & 2 & 2 & 0 & 0 & 0 & 0 & 0 & 0 & 0 \\
\hline $\mathrm{A} 16$ & $\mathrm{H} 4$ & 0 & 0 & 3 & 4 & 4 & 2 & 2 & 0 & 0 & 0 & 0 & 0 & 0 & 0 \\
\hline $\mathrm{A} 16$ & $\mathrm{DH}$ & 0 & 0 & 0 & 0 & 0 & 0 & 0 & 5 & 5 & 5 & 5 & 5 & 5 & 6 \\
\hline
\end{tabular}

Table 26. Allocation of patient type 2 for 6 -week pandemic duration, $35 \%$ gross attack rate, most likely scenario (day 1-14)

\begin{tabular}{|c|c|c|c|c|c|c|c|c|c|c|c|c|c|c|c|}
\hline & $\mathrm{X}$ & $\mathrm{D} 1$ & $\mathrm{D} 2$ & $\mathrm{D} 3$ & $\mathrm{D} 4$ & $\mathrm{D} 5$ & $\mathrm{D} 6$ & $\mathrm{D} 7$ & $\mathrm{D} 8$ & $\mathrm{D} 9$ & $\mathrm{D} 10$ & $\mathrm{D} 11$ & $\mathrm{D} 12$ & $\mathrm{D} 13$ & $\mathrm{D} 14$ \\
\hline A5 & H5 & 0 & 0 & 0 & 0 & 0 & 0 & 0 & 0 & 0 & 0 & 0 & 1 & 1 & 1 \\
\hline A6 & H1 & 0 & 0 & 0 & 0 & 1 & 1 & 1 & 1 & 1 & 1 & 1 & 1 & 1 & 1 \\
\hline A11 & H3 & 0 & 0 & 0 & 0 & 0 & 0 & 0 & 0 & 0 & 0 & 1 & 1 & 1 & 1 \\
\hline A14 & H1 & 0 & 0 & 0 & 0 & 0 & 0 & 0 & 1 & 1 & 1 & 1 & 1 & 1 & 1 \\
\hline
\end{tabular}

Table 27. Allocation of patient type 3 for 6 -week pandemic duration, $35 \%$ gross attack rate, most likely scenario (day 1-14)

\begin{tabular}{|c|c|c|c|c|c|c|c|c|c|c|c|c|c|c|c|}
\hline & $\mathrm{X}$ & $\mathrm{D} 1$ & $\mathrm{D} 2$ & $\mathrm{D} 3$ & $\mathrm{D} 4$ & $\mathrm{D} 5$ & $\mathrm{D} 6$ & $\mathrm{D} 7$ & $\mathrm{D} 8$ & $\mathrm{D} 9$ & $\mathrm{D} 10$ & $\mathrm{D} 11$ & $\mathrm{D} 12$ & $\mathrm{D} 13$ & $\mathrm{D} 14$ \\
\hline $\mathrm{A} 5$ & $\mathrm{H} 5$ & 0 & 0 & 0 & 0 & 0 & 0 & 0 & 0 & 0 & 0 & 0 & 1 & 0 & 0 \\
\hline $\mathrm{A} 5$ & $\mathrm{DH}$ & 0 & 0 & 0 & 0 & 0 & 0 & 0 & 0 & 0 & 0 & 0 & 0 & 1 & 1 \\
\hline $\mathrm{A} 6$ & $\mathrm{H} 2$ & 0 & 0 & 0 & 0 & 1 & 0 & 0 & 0 & 1 & 1 & 0 & 0 & 0 & 0 \\
\hline $\mathrm{A} 6$ & $\mathrm{H} 4$ & 0 & 0 & 0 & 0 & 0 & 1 & 1 & 1 & 0 & 0 & 1 & 1 & 0 & 0 \\
\hline $\mathrm{A} 6$ & $\mathrm{DH}$ & 0 & 0 & 0 & 0 & 0 & 0 & 0 & 0 & 0 & 0 & 0 & 0 & 1 & 1 \\
\hline $\mathrm{A} 11$ & $\mathrm{H} 3$ & 0 & 0 & 0 & 0 & 0 & 0 & 0 & 0 & 0 & 0 & 0 & 0 & 1 & 0 \\
\hline $\mathrm{A} 11$ & $\mathrm{H} 7$ & 0 & 0 & 0 & 0 & 0 & 0 & 0 & 0 & 0 & 0 & 1 & 1 & 0 & 0 \\
\hline $\mathrm{A} 11$ & $\mathrm{DH}$ & 0 & 0 & 0 & 0 & 0 & 0 & 0 & 0 & 0 & 0 & 0 & 0 & 0 & 1 \\
\hline $\mathrm{A} 14$ & $\mathrm{H} 1$ & 0 & 0 & 0 & 0 & 0 & 0 & 0 & 0 & 0 & 0 & 1 & 0 & 0 & 0 \\
\hline $\mathrm{A} 14$ & $\mathrm{H} 5$ & 0 & 0 & 0 & 0 & 0 & 0 & 0 & 0 & 1 & 0 & 0 & 0 & 0 & 0 \\
\hline $\mathrm{A} 14$ & $\mathrm{H} 6$ & 0 & 0 & 0 & 0 & 0 & 0 & 0 & 1 & 0 & 1 & 0 & 1 & 0 & 0 \\
\hline $\mathrm{A} 14$ & $\mathrm{DH}$ & 0 & 0 & 0 & 0 & 0 & 0 & 0 & 0 & 0 & 0 & 0 & 0 & 1 & 1 \\
\hline
\end{tabular}


Table 28. Allocation of patient type 1 with additional resource allocation for 6-week pandemic duration, $35 \%$ gross attack rate, most likely scenario (day 1-14)

\begin{tabular}{|l|l|r|r|r|r|r|r|r|r|r|r|r|r|r|r|}
\hline & $\mathrm{X}$ & $\mathrm{D} 1$ & $\mathrm{D} 2$ & $\mathrm{D} 3$ & $\mathrm{D} 4$ & $\mathrm{D} 5$ & $\mathrm{D} 6$ & $\mathrm{D} 7$ & $\mathrm{D} 8$ & $\mathrm{D} 9$ & $\mathrm{D} 10$ & $\mathrm{D} 11$ & $\mathrm{D} 12$ & $\mathrm{D} 13$ & $\mathrm{D} 14$ \\
\hline $\mathrm{A} 1$ & $\mathrm{H} 5$ & 1 & 1 & 1 & 1 & & 2 & 2 & & & & & & & \\
\hline $\mathrm{A} 1$ & $\mathrm{H} 6$ & & & & & 2 & & & 2 & 2 & 2 & 2 & 2 & 2 & 2 \\
\hline $\mathrm{A} 2$ & $\mathrm{H} 2$ & 2 & 2 & 2 & 2 & 2 & 2 & 2 & 3 & 3 & & & & & \\
\hline $\mathrm{A} 2$ & $\mathrm{H} 6$ & & & & & & & & & & 3 & 3 & 3 & 3 & 4 \\
\hline $\mathrm{A} 3$ & $\mathrm{H} 6$ & 1 & 1 & 2 & 2 & 2 & 2 & 2 & 2 & 2 & 2 & 2 & 2 & 2 & 2 \\
\hline $\mathrm{A} 4$ & $\mathrm{H} 4$ & 2 & 2 & 2 & 3 & 3 & 3 & 3 & 3 & 3 & 4 & 4 & 4 & 4 & 4 \\
\hline A5 & $\mathrm{H} 5$ & 4 & 4 & 4 & 4 & 4 & 4 & 4 & 5 & 5 & 4 & 6 & 6 & 4 & 6 \\
\hline A5 & $\mathrm{H} 6$ & & & & & & & & & & 1 & & & 2 & \\
\hline A6 & $\mathrm{H} 1$ & 5 & 5 & 5 & 6 & 6 & 6 & 6 & 7 & 7 & 8 & 8 & 8 & 8 & 9 \\
\hline A7 & $\mathrm{H} 1$ & 2 & 2 & 2 & 2 & 2 & 3 & 3 & 3 & 3 & 3 & 3 & 3 & 4 & 4 \\
\hline A8 & H2 & 2 & 2 & 2 & 2 & 2 & 2 & 2 & 3 & 3 & 1 & 3 & 2 & 3 & 3 \\
\hline A8 & H6 & & & & & & & & & & 2 & & 1 & & \\
\hline A9 & H2 & 2 & 2 & 2 & 2 & 2 & 2 & 2 & 3 & 3 & 3 & 3 & 3 & 3 & 3 \\
\hline A10 & H1 & 1 & 2 & 2 & 2 & 2 & 2 & 2 & 2 & 2 & & & 1 & & \\
\hline A10 & H2 & & & & & & & & & & 2 & 2 & 1 & 2 & 3 \\
\hline A11 & H3 & 4 & 4 & 4 & 4 & 4 & 4 & 4 & 5 & 5 & 6 & 6 & 6 & 6 & 6 \\
\hline A12 & H7 & 3 & 3 & 3 & 3 & 3 & 3 & 3 & 4 & 4 & 4 & 4 & 4 & 4 & 4 \\
\hline A13 & H7 & 3 & 3 & 3 & 3 & 3 & 3 & 3 & 4 & 4 & 4 & 4 & 4 & 5 & 5 \\
\hline A14 & H1 & 4 & 4 & 4 & 4 & 5 & 5 & 5 & 6 & 6 & 6 & 6 & 7 & 7 & 7 \\
\hline A15 & H2 & & & & & & & & & & & & & 1 & \\
\hline A15 & H3 & 3 & 3 & 3 & 3 & 3 & 4 & 4 & 4 & 4 & 1 & 4 & 5 & 4 & 5 \\
\hline A15 & H4 & & & & & & & & & & 4 & 1 & & & \\
\hline A16 & H3 & 3 & 3 & 3 & 4 & & 3 & 2 & 1 & 2 & & & & & \\
\hline A16 & H4 & & & & & 4 & 1 & 2 & 4 & 3 & 5 & 5 & 5 & 5 & 6 \\
\hline
\end{tabular}

Table 29. Allocation of patient type 2 with additional resource allocation for 6-week pandemic duration, $35 \%$ gross attack rate, most likely scenario (day 1-14)

\begin{tabular}{|l|l|l|l|l|l|r|r|r|r|r|r|r|r|r|r|}
\hline & X & D1 & D2 & D3 & D4 & D5 & D6 & D7 & D8 & D9 & D10 & D11 & D12 & D13 & D14 \\
\hline A5 & H5 & & & & & & & & & & & & 1 & 1 & 1 \\
\hline A6 & H1 & & & & & & & & & 1 & 1 & 1 & 1 & 1 & \\
\hline A6 & H2 & & & & & 1 & 1 & 1 & 1 & & & & & & 1 \\
\hline A11 & H3 & & & & & & & & & & & 1 & 1 & 1 & 1 \\
\hline A14 & H1 & & & & & & & & 1 & 1 & 1 & 1 & 1 & 1 & 1 \\
\hline
\end{tabular}


Table 30. Allocation of patient type 3 with additional resource allocation for 6 -week pandemic duration, $35 \%$ gross attack rate, most likely scenario (day 1-14)

\begin{tabular}{|l|l|l|l|l|l|l|l|r|r|r|r|r|r|r|r|}
\hline & X & D1 & D2 & D3 & D4 & D5 & D6 & D7 & D8 & D9 & D10 & D11 & D12 & D13 & D14 \\
\hline A5 & H5 & & & & & & & & & & & & 1 & & 1 \\
\hline A5 & H6 & & & & & & & & & & & & & 1 & \\
\hline A6 & H1 & & & & & & & & & & & & & & 1 \\
\hline A6 & H2 & & & & & & & & & 1 & & 1 & 1 & & \\
\hline A6 & H4 & & & & & 1 & 1 & 1 & & & & & & 1 & \\
\hline A6 & H6 & & & & & & & 1 & & 1 & & & & \\
\hline A11 & H3 & & & & & & & & & & & & 1 & \\
\hline A11 & H4 & & & & & & & & & & & & & 1 \\
\hline A11 & H7 & & & & & & & & & & & 1 & 1 & & \\
\hline A14 & H1 & & & & & & & & 1 & 1 & 1 & 1 & 1 & 1 & 1 \\
\hline
\end{tabular}




\section{CURRICULUM VITAE}

Name: $\quad$ Li Sun

Address: $\quad$ Department of Industrial Engineering

J.B. Speed School of Engineering

University of Louisville

Louisville, KY 40292

Education: Bachelor of Engineering, Mechanical and Electrical Engineering

Nanjing University of Aeronautics and Astronautics

1999-2003

Master of Engineering, Mechanical and Electrical Engineering

Nanjing University of Aeronautics and Astronautics

2003-2006

Master of Science, Industrial Engineering

University of Louisville

2007-2008

PHD, Industrial Engineering

University of Louisville

Defended on Dec 9, 2011

Professional Societies and Memberships:

Institute of Industrial Engineers

The Institute for Operations Research and the Management Sciences 